\title{
Development of Eccentric Black Hole Binary Searches in the LIGO and PTA Regimes
}

\author{
Belinda D. Cheeseboro \\ West Virginia University, bdc0001@mix.wvu.edu
}

Follow this and additional works at: https://researchrepository.wvu.edu/etd

Part of the Cosmology, Relativity, and Gravity Commons

\section{Recommended Citation}

Cheeseboro, Belinda D., "Development of Eccentric Black Hole Binary Searches in the LIGO and PTA Regimes" (2021). Graduate Theses, Dissertations, and Problem Reports. 10269.

https://researchrepository.wvu.edu/etd/10269

This Dissertation is protected by copyright and/or related rights. It has been brought to you by the The Research Repository @ WVU with permission from the rights-holder(s). You are free to use this Dissertation in any way that is permitted by the copyright and related rights legislation that applies to your use. For other uses you must obtain permission from the rights-holder(s) directly, unless additional rights are indicated by a Creative Commons license in the record and/ or on the work itself. This Dissertation has been accepted for inclusion in WVU Graduate Theses, Dissertations, and Problem Reports collection by an authorized administrator of The Research Repository @ WVU.

For more information, please contact researchrepository@mail.wvu.edu. 


\title{
Development of Eccentric Black Hole Binary Searches in the LIGO and PTA Regimes
}

\author{
Belinda D. Cheeseboro
}

\author{
Dissertation Submitted to \\ The Eberly College of Arts and Sciences \\ at West Virginia University \\ in partial fulfillment of the requirements \\ for the degree of
}

Doctor of Philosophy

in

Physics
Sarah Burke-Spolaor, Ph.D., Chair
Maura Mclaughlin, Ph.D.
Paul Baker, Ph.D.
Tiffany Summerscales, Ph.D.
Morgantown, West Virginia, USA
2021

Keywords: gravitational waves, eccentricity, binaries

Copyright 2021 Belinda D. Cheeseboro 


\title{
Abstract \\ Development of Eccentric Black Hole Binary Searches in the LIGO and PTA Regimes
}

\author{
Belinda D. Cheeseboro
}

In the past several years, a plethora of gravitational wave events have been detected leading to better understanding of binary black holes, binary neutron stars, and neutron star black hole binaries. All of these transient detections have helped us better understand the dynamics of these systems as well as the populations of these objects, but each of these sources was detected with models that neglected eccentricity. Eccentricity is one of several potential markers for determining the formation of binary systems. Detecting gravitational waves from eccentric sources can better our understanding of such systems and help constrain theories about their formation. In the ground-based gravitational-wave regime, most eccentric binary black hole sources will be detected with little to no eccentricity $(e<0.1)$ as they enter the detectable frequency band. There are sources that could enter the frequency band with higher eccentricity $(e>0.1)$, but the lack of eccentricity-based models implemented into current search methods will make detecting such systems difficult. In the pulsar timing array regime, previous implementations of eccentricitybased models proved to be too computationally expensive. Recent developments in eccentric modeling of supermassive black hole binary systems have made it possible to incorporate eccentricity in a search for continuous gravitational waves from eccentric supermassive black hole binary sources. This work details the methods developed to aid in searching for eccentric stellar-mass black hole binary sources in the ground-based gravitational-wave regime and eccentric supermassive black hole binaries in the pulsar timing array regime. 


\section{Acknowledgements}

I would like to thank the West Virginia University Department of Graduate Education and Life for recognition as a STEM Mountains of Excellence Fellow. This fellowship is funded by a grant provided by the West Virginia Higher Education Policy Commission's Division of Science and Research. I would also like to thank the Chancellor's Scholar Program at WVU and the CIFAR Azrieli Global Scholarship in Gravity \& the Extreme Universe. I would also like to note that I received partial support from National Science Foundation (NSF) Physics Frontier Center award \#1430284.

As for the people involved in making this work possible, I'd first like to thank my advisor, Sarah Burke-Spolaor, for taking me on as a graduate student late into my graduate career. Although I wanted to give up many times, she encouraged me to keep moving forward. Next I'd like to give a big thanks to my collaborator, Paul Baker, for teaching me everything that I needed to know to get our LIGO project done. Without him our project would have never gotten off the ground. I'd also like to acknowledge my collaborators for my PTA project for their help as problems arose. Next are the faculty from the physics, math, and computer science departments of Andrews University. Through their care and tutelage I was able to learn so much and this knowledge formed the foundation of the student that I am today. Next I want to give a shout-out to my family and friends who never stopped believing in me when I wanted to give up on myself, especially my best friends Amber Lenon and Megan Jones. Amber and Megan have consistently been there for me when the going got tough. Without them I would not have been able to survive the difficult process of attaining a Ph.D. Lastly I want to acknowledge my late father, Amos Cheeseboro. He instilled the love of science and learning within me and without him I would not have started my science journey. 


\section{Table of Contents}

List of Figures $\quad$ vi

1 Introduction 1

1.1 Formation of Binary Black Hole Sources . . . . . . . . . . . . . . . . 2

1.1.1 Stellar-Mass Black Hole Binaries . . . . . . . . . . . . . . . . 2

1.1.2 Supermassive Black Hole Binaries . . . . . . . . . . . . . . . . 4

1.2 Eccentric GWs . . . . . . . . . . . . . . . . . . 7

1.2.1 Eccentricity . . . . . . . . . . . . . 8

1.2.2 Properties of Eccentric GWs . . . . . . . . . . . . . . . 10

2 Detection of GWs 18

2.1 GW Detectors . . . . . . . . . . . . . . . . . . . . . 18

2.1.1 Ground-Based Detectors . . . . . . . . . . . . . 20

2.1.1.1 Search Methods . . . . . . . . . . . . . . . 24

2.1.1.2 The Status of Detections by Ground-based Detectors 28

2.1.2 Pulsar Timing Arrays . . . . . . . . . . . . . . . . 31

2.1.2.1 Pulsar Timing Data . . . . . . . . . . . . . 34

2.2 Motivation and Challenges of Detecting Eccentric BBH Sources . . . 40

2.2.1 In the Ground-Based Regime . . . . . . . . . . . . . . . . . . 40

2.2.2 In the PTA Regime . . . . . . . . . . . . . . . . . 42

3 Method for Detecting Highly-Eccentric Binaries with a Gravitational-Wave Burst Search 46

3.1 Initial Testing with BayesWave . . . . . . . . . . . . . . . . . . . . 47

3.2 Modeling Bursts from Highly Eccentric Sources . . . . . . . . . . . . 49

3.2.1 Centroid Mapping Equations . . . . . . . . . . . . . 51

3.2.2 Eccentric Burst Prior . . . . . . . . . . . . . . . . . 54

3.2 .3 Signal Model ................... . . 56

3.3 Analysis of Simulated Data . . . . . . . . . . . . . . 58

3.3.1 Simple Wavelet Injections . . . . . . . . . . . . . . . . . . . 61

3.3.2 Eccentric BBH Waveform Injections . . . . . . . . . . . . . . 64

3.4 Discussion and Conclusions . . . . . . . . . . . . . 66

3.5 Appendix .......................... 71

4 Implementing an Eccentricity Search for Pulsar Timing Arrays 74

4.1 Introduction . . . . . . . . . . . . . . . . . . . . 74

4.2 Data and Methods . . . . . . . . . . . . . . . . . . . . . . . . . . . . . . . . . 77

4.2.1 Pulsar Timing datasets . . . . . . . . . . . . . . . . . 77

4.2.2 Signal Model . . . . . . . . . . . . . . . . . 78

4.2 .3 Software ..................... . . . 82

4.2.4 Creation of the Simulated Dataset . . . . . . . . . . . . . 83

4.2.5 Procedure for Detecting and Limiting GWs . . . . . . . . . . 84

4.2.6 Detection Statistic . . . . . . . . . . . . . . . . 87 
4.3 Results and Discussion . . . . . . . . . . . . . . . . . . . 90

4.3.1 Injection Test Results . . . . . . . . . . . . . . . . . 90

4.3.2 Application of the Eccentric Search Method on Real Data . . 91 4.3.2.1 An Illustrative Example of Code Considerations: Binary Candidate 3C 66B . . . . . . . . . . . 92

4.3.2.2 GW search for a 3C 66B-Like Source . . . . . . . . 97

4.4 Conclusions . . . . . . . . . . . . . . . . . . 106

5 Conclusion 108

5.1 Eccentric Burst Prior: Caveats and Future Improvements . . . . . . . 109

5.2 Implementation of an Eccentric GW Search on PTA Data . . . . . 110

5.2.1 Limits on Eccentricity and Chirp Mass . . . . . . . . . . . 111

5.2.2 Future Improvements to Eccentric Searches on PTA Datasets 111

5.3 The Future of Eccentric Gravitational-Wave Searches . . . . . . . . 112

5.3 .1 Ground-Based Detectors . . . . . . . . . . . . . . . . . 112

5.3 .2 Pulsar Timing Arrays . . . . . . . . . . . . . . . . . . 113 


\section{List of Figures}

1.1 Graphic depicting the process for a common envelope phase and a three body encounter for a stellar-mass BBH system . . . . . . . . . 3

1.2 Formation of an SMBHB . . . . . . . . . . . . . 5

1.3 Schematic showing the shape of an orbit varying with eccentricity. . . 8

1.4 Sketch of an ellipse with object at one of the foci. . . . . . . . . 9

1.5 Figures showing the decay of the semi-major axis and eccentricity for the Hulse-Taylor binary as a function of time (years). . . . . . . . . 11

1.6 The difference in signals from a circular source vs an eccentric source. 14

1.7 Relative power for specific eccentricities over different frequency harmonics. . . . . . . . . . . . . . . . . . . . . 15

2.1 GW spectrum with types of detectors. . . . . . . . . . . . 19

2.2 Basic setup of a Michelson interferometer. . . . . . . . . . . . . 22

2.3 Sensitivity curves for the currently active ground-based GW detectors. 23

2.4 BayesWave model selection . . . . . . . . . . . . . . . . 27

2.5 GW transients from the open GW catalog of aLIGO and Virgo observing runs during 2015-2019. These are shown in time-frequency space. . . . . . . . . . . . . . . . . . . 30

2.6 Hellings \& Downs curve . . . . . . . . . . . . . . . . . . 33

2.7 Schematic showing the process of timing a pulsar . . . . . . . 35

2.8 The effect of different astrophysical errors on the timing residual of a

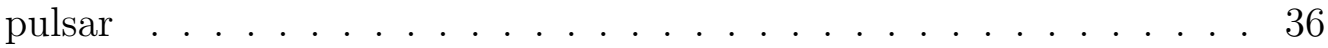

2.9 Simulated noise curve based on the 12.5 year dataset including the white noise parameters. . . . . . . . . . . . . . . 38

2.10 Residuals from a quasi-circular source vs an eccentric source for both polarization modes. . . . . . . . . . . . . . . . . . . . 43

2.11 Simulated characteristic strain spectra for different stochastic background populations. . . . . . . . . . . . . . . . . . . 44

3.1 Simulated eccentric GW signal recovered with BayesWave . . . . . . . 48

3.2 Prior probability as a function of time-frequency location. . . . . . . . 56

3.3 Parameter estimation uncertainty for the simple wavelet and eccentric BBH waveform injections. . . . . . . . . . . . . . 63

3.4 Corner plot showing the posterior distributions for the eccentric burst prior meta parameters for a case with SNR per Burst $=4 \ldots \ldots 68$

4.1 Waveform injected into simulated data. . . . . . . . . . . . 88

4.2 Posterior distributions for global search parameters from injection analysis. . . . . . . . . . . . . . . . . . . 89 
4.3 This figure represents specifically the source $3 \mathrm{C} 66 \mathrm{~B}$, where the restframe orbital period of 1.07 years is placed at a reference epoch of Gregorian calendar year 2003. For systems at or above the lines shown, a binary of the given chirp mass and eccentricity will merge before the end of the NANOGrav data sets searched in this paper. . . 95

4.4 Residuals of 3C 66B at different eccentricities. . . . . . . . . . . . . 98

4.5 Corner plot showing 1-D and 2-D distributions for $e_{0}$ and $\log _{10}(\mathcal{M})$ using the posterior samples obtained from the analysis done on the NANOGrav 12.5-year dataset. . . . . . . . . . . . . . . . 100

4.6 Corner plot showing 1-D and 2-D distributions for $e_{0}$ and $\log _{10}(\mathcal{M})$ using the posterior samples from the analysis on the NANOGrav 11year dataset. . . . . . . . . . . . . . . . . . . . . . 101 


\section{Chapter 1}

\section{Introduction}

The Universe is filled with many wonders that have yet to be discovered and explained by science. Of the many wonders in the Universe, black holes are an astrophysical phenomena that are simple in construction yet defy the boundaries of our imagination. They are points in spacetime that trap light due to their extreme gravity. Studying these fascinating objects allows us to test the predictions of general relativity and probe the black hole population. GWs detected by ground-based detectors have provided a wealth of information that has allowed us to perform these tests of general relativity and expand our understanding of the black hole population. With further detections from ground-based detectors and future detections from pulsar timing arrays we will gain a better understanding of the formation of binary black holes. Currently, the formation of binary black holes is still uncertain and requires improvements to the search methods utilized by GW detectors. Through the detection of GWs from eccentric binary black hole (BBH) sources we may be able to gain insight into their formation. This chapter will first provide an overview of the current understanding of the evolution of binary black hole sources; then a general overview of the properties of GWs and eccentricity. 


\subsection{Formation of Binary Black Hole Sources}

To understand the formation of BBH sources, one must start from the stars that formed them. Stars form from the gravitational collapse of a cloud of dense, cold gas and dust. Depending on the amount of gas and dust, stars can have masses ranging from less than a 10th of a solar mass to hundreds of solar masses (Scalo 1986). The population of stellar-mass black holes $\left(5-100 M_{\odot}\right)$ is formed from

high-mass stars $\left(M>18 M_{\odot}\right.$, Ryden \& Peterson 2020) going supernova due to the collapse of their cores at the end of their lives.

\subsubsection{Stellar-Mass Black Hole Binaries}

Stellar-mass black hole binary sources can form in several different ways but can be categorized as either forming 1) in an isolated environment or 2) in a dynamic environment. An isolated environment is one in which the binary evolves unperturbed by other sources whereas a binary in a dynamic environment is fully susceptible to the influences of the environment of the binary. The most quoted scenario for a binary formed in an isolated environment is that of the "common envelope" phase. The common envelope phase is typically where the more massive companion leaves the main sequence phase and its hydrogen layer expands and envelops the less massive companion. 

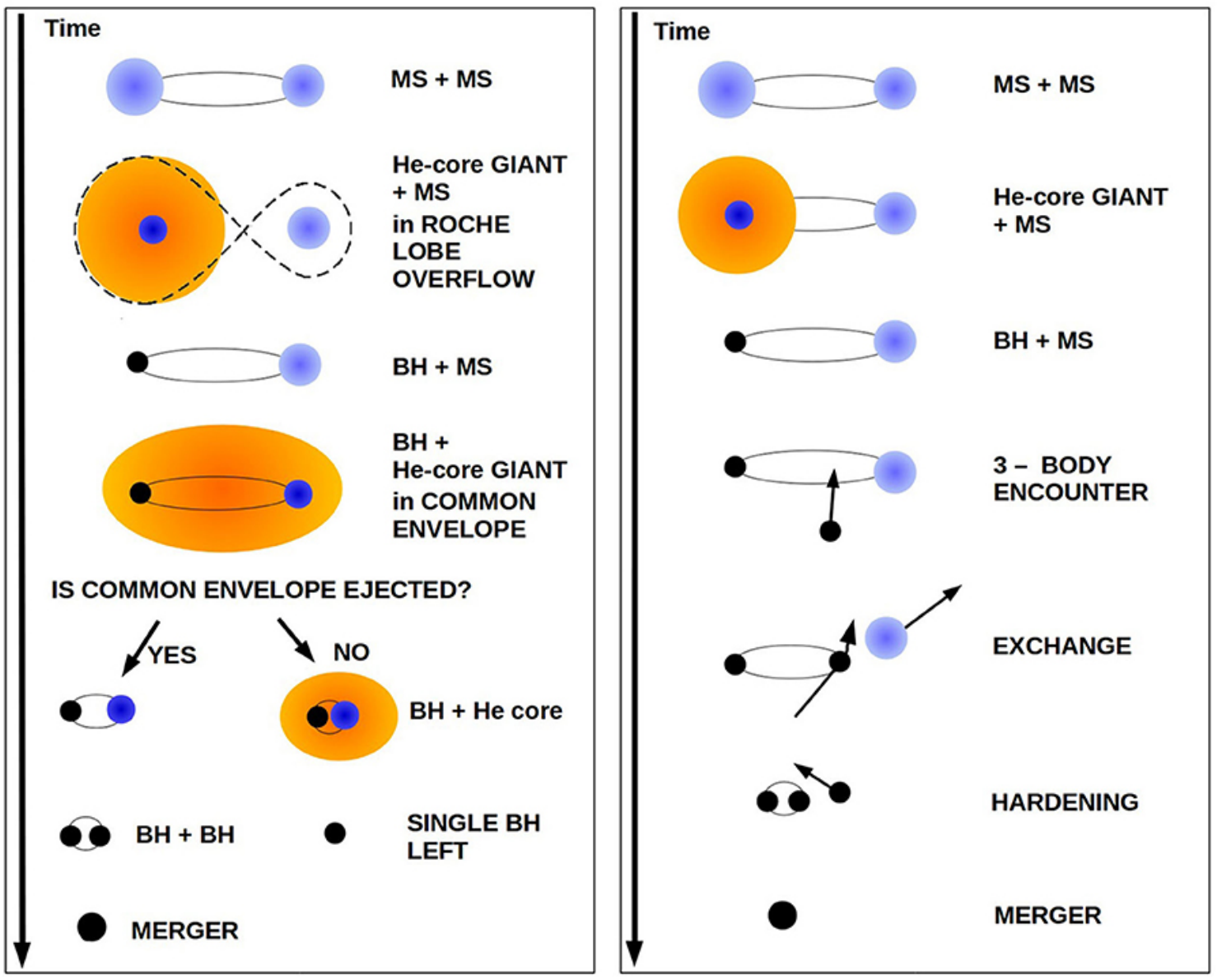

Figure 1.1: Graphic depicting the process for common envelope phase and three body encounter for a stellar-mass BBH system (Mapelli, 2020). left: Depicts a stellar binary going through the common envelope phase. If the common envelope is ejected, then a BBH will form. Otherwise the companion black hole will be absorb the helium core, resulting in a single black hole. This scenario is typical for binaries that form in isolated environments. right: Depicts the process of a threebody encounter. After one of the companions in the binary becomes a black bole, another black hole may come in and take the place of the stellar binary companion. The stellar binary companion eventually becomes a black hole and through it's interactions with the $\mathrm{BBH}$ system, it causes the BBH system to gravitationally harden as it is being ejected. This scenario is typical for BBHs that form in dynamic environments.

As the more massive companion sheds its hydrogen layer through mass-transfer to the less massive companion, still in the main sequence phase, it begins the process of helium burning until its core collapses causing a supernova. This core will collapse 
into either a black hole or neutron star. The less massive companion will also go through the same process that the more massive companion experienced. As the newly formed compact object sits in the envelope of the companion, there are two possible outcomes of this process. The mass transfer of the less massive star is unstable, resulting in the companions sharing an envelope. From this the compact object will spiral into the dense stellar environment, caused by the shared envelope, shrinking the orbit of the binary. After the inspiral phase there are two typical outcomes. If the less massive star manages to release its envelope and the system survives the supernova explosion, then the binary will merge within the lifetime of the universe (Paczynski, 1976). If it doesn't manage to shed this envelope then the compact object and secondary star could merge with its companion and become a single compact object. This process is illustrated in the left panel of Figure 1.1.

For stellar-mass BBH sources that form in dynamic environments, like globular clusters or galactic nuclei, they undergo many interactions with nearby objects before becoming tightly bound by gravity and forming a pair (Banerjee et al., 2010; Banerjee, 2018a,b). This process is illustrated in the right panel of Figure 1.1. stellar-mass $\mathrm{BBHs}$ that form in dynamic environments will be discussed more in the next section.

\subsubsection{Supermassive Black Hole Binaries}

Supermassive black holes, with masses $\left(M>10^{6} M_{\odot}\right)$, have distinctly different formation scenarios than stellar-mass black holes, as their gargantuan size requires 
different physical processes to make them. The first being the "direct collapse model" where a supermassive black hole is formed from the collapse of a massive gas cloud (Rees, 1984) and can grow through the accretion of gas and other black holes. The second assumes a supermassive black hole started as a stellar-mass black hole that accreted gas and grew to be supermassive (Rees, 1984). This theory is no longer popular as there is evidence of SMBHs existing at high redshifts (Paliya et al., 2019). The last is the idea of "seed black holes" which are primordial black holes with masses on the order of $1000 M_{\odot}$ that could have been the start of the supermassive black holes we know today (Latif \& Ferrara, 2016). Seed black holes are the most popular theory as the progenitors of the SMBHs. While this population of black holes will not be covered in this work, it is worth mentioning that they will be probed by future space-based endeavors like LISA (Amaro-Seoane et al., 2017).

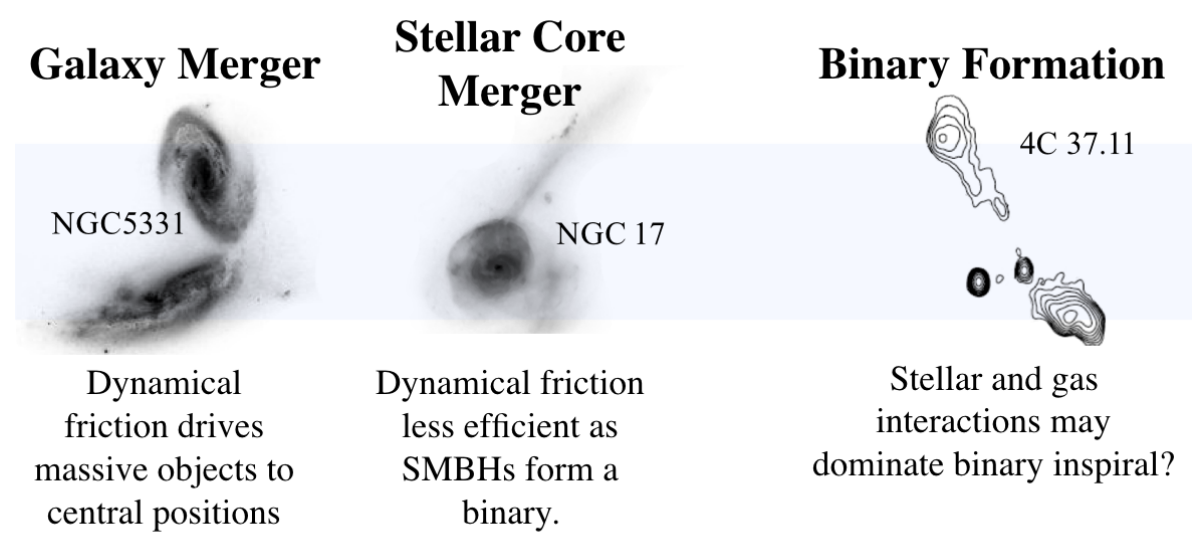

Figure 1.2: Graphic depicting the formation of a SMBHB. In the first part of the graphic, two galaxies begin to merge through the process of dynamical friction. Through mass segregation, the cores of both galaxies fall to the center of the gravitational potential well and also begin to merge. Within the merged core, more dynamical interactions with stars and gas results in the formation of a SMBHB. Adapted from figure 3 of Burke-Spolaor et al. (2019). 
Supermassive black hole binaries (SMBHBs) are the by-product of the collision of two galaxies. When two galaxies interact, gravitational interactions between stars, gas, and resident black holes drive the whole system to become gravitationally bound as a merger remnant (Figure 1.2); this can also lead some stars getting thrown out entirely. As more passages occur, more gas, dust, and stars get stripped from one galaxy and collect onto the other. These interactions causes the constituent galaxies to slow down and get closer to each other. Over time the two galaxies merge to form a larger galaxy along with their cores. Most massive galaxies contain a supermassive black hole in their cores (Kormendy \& Richstone, 1995). As the cores of the constituent galaxies are in the process of merging, their supermassive black holes can also interact.

These supermassive black holes form a pair, dragged preferentially to the center of the remnant by dynamical friction, which describes the interaction between the binary and the residual gas, dust, and stars from the collision (Begelman et al., 1980). The process by which the largest masses preferentially come to the merger's center is termed mass segregation. Mass segregation is where the heavier objects in a galaxy will move towards the lowest part of the galaxy's gravitational potential well. Over time this binary will harden, meaning its orbit will shrink by the process of exchanging orbital angular momentum with stars and gas in its environment.

At this stage, it becomes a question of whether the binary will eventually merge, or be destined to dance with each other for all eternity. The main issue is knowing what mechanism(s) drives the binary to merge. Simulations have shown that dynamical friction alone cannot drive a supermassive black hole binary to 
merger (Quinlan, 1996), and GWs will be the dominant source of emission only if the separation of the binary gets within 1/10 pc (Begelman et al., 1980). This issue is known as the final parsec problem. The detection of GWs from SMBHBs could give more insight into how these binaries merge within the age of the Universe.

\subsection{Eccentric GWs}

Albert Einstein first theorized about the existence of gravitational waves (GWs) in 1916 after the development of his theory of General Relativity. He postulated that gravitational radiation would occur when a gravitating system's quadrupole moment, which describes the motion of the system, is accelerated. The resultant amplitude of these waves would be extremely small and therefore too difficult to detect with the instruments of his time. Little did he know that 100 years later, his prediction would be proven correct via the detection of GWs from a BBH system that merged just over 1.3 billion years ago (Abbott et al., 2016a). Through this detection, we were ushered into a new age of astronomy were we could uniquely probe the dynamics of binary black hole systems using this novel cosmic messenger. In this section we will primarily focus on eccentric GWs and how they can inform us of the formation of a binary. 


\subsubsection{Eccentricity}

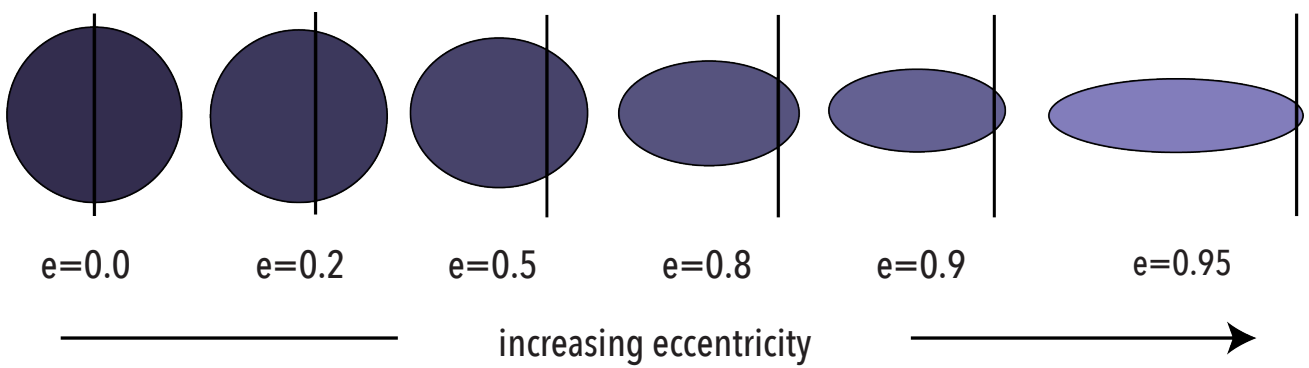

Figure 1.3: Schematic showing the shape of an orbit varying with eccentricity. The vertical line in each ellipse indicates one of the two foci. Adapted from Swinburne University of Technology.

Eccentricity is a parameter that describes how much an orbit deviates from a perfect circle (see Figure 1.3). The motion of a particle in an elliptical orbit is described by the orbital equation from Kepler's First Law

$$
r(\theta)=\frac{a\left(1-e^{2}\right)}{1+e \cos (\theta)},
$$

where $r(\theta)$ tracks the position of the particle in its orbit assuming the origin is at one of the foci of the ellipse, $a$ is the semi-major axis of the orbit, $e$ is the eccentricity of the orbit, and $\theta$ is the true anomaly (see Figure 1.4). The true anomaly is the angle between the point of periapsis (closest point to the body that is being orbited) and the current position of the particle in its orbit. This law shows that in the presence of Newtonian gravity, a particle would have this orbital motion around a gravitating body.

In the case of general relativity, this picture begins to break down. Kepler's 


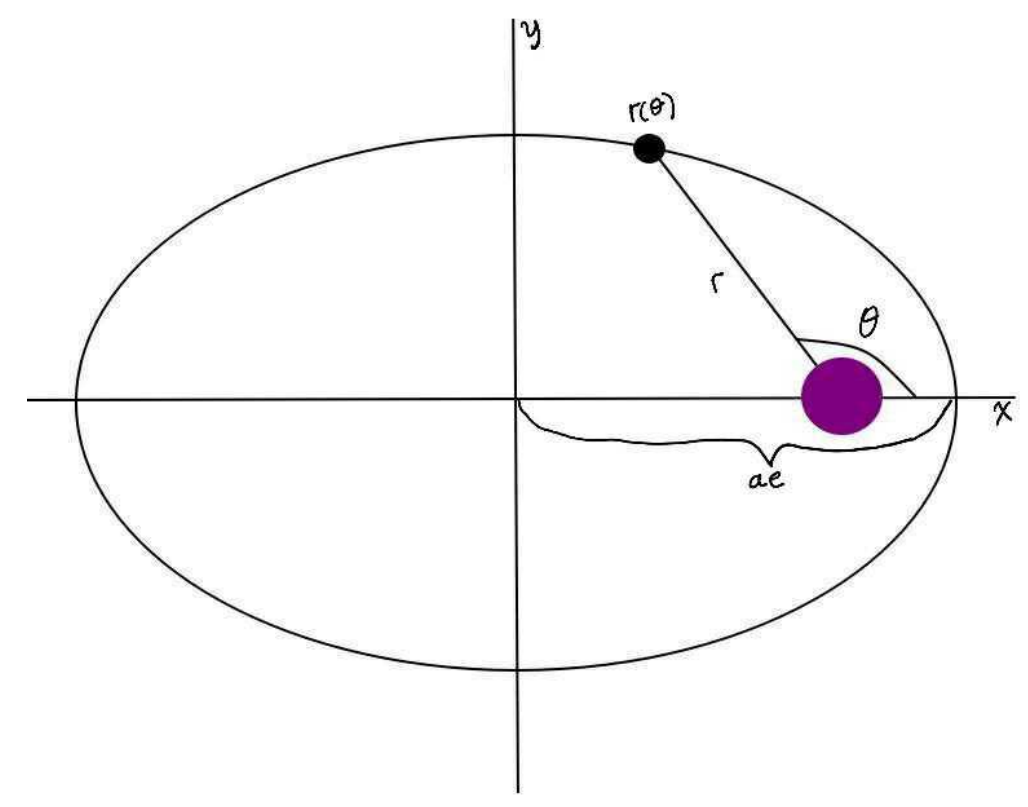

Figure 1.4: Sketch of an ellipse with object at one of the foci.

Laws assume closed orbits, this law breaks in the presence of warped space-time. This is seen in the classic observation of the precession of Mercury's orbit around the Sun (Bramanti, 1968). Kepler's Laws are good approximations for all the other planets' orbits, however Mercury's proximity to the Sun meant that it was orbiting in significantly warped space-time. This was a mystery to astronomers until Einstein developed his theory of general relativity. The theory of general relativity provides prescriptions for how matter behaves in warped spacetime, and it was this theory that helped Einstein solve the mystery of Mercury's orbit. It was also through the development of general relativity that Einstein postulated about gravitational radiation or GWs. 


\subsubsection{Properties of Eccentric GWs}

Eccentric BBH systems create an impressive display of orbital dynamics. Based on general relativistic binary evolution (i.e. an orbit driven purely by emission of GWs), one can calculate the orbital decay for the semi-major axis and orbital eccentricity. As derived by (Peters, 1964), the orbital evolution can be described by the following time-averaged coupled differential equations

$$
\left\langle\frac{d a}{d t}\right\rangle=-\frac{64}{5} \frac{G^{4}}{c^{5}} \frac{m_{1}^{2} m_{2}^{2}\left(m_{1}+m_{2}\right)}{a^{3}\left(1-e^{2}\right)^{7 / 2}}\left(1+\frac{73}{24} e^{2}+\frac{37}{96} e^{4}\right),
$$

and

$$
\left\langle\frac{d e}{d t}\right\rangle=-\frac{304}{15} e \frac{G^{4}}{c^{5}} \frac{m_{1}^{2} m_{2}^{2}\left(m_{1}+m_{2}\right)}{a^{4}\left(1-e^{2}\right)^{5 / 2}}\left(1+\frac{121}{304} e^{2}\right),
$$

where $G$ is the gravitational constant, $c$ is the speed of light, $m_{1}$ and $m_{2}$ are the component masses, and as previously, $a$ is the semi-major axis, and $e$ is the orbital eccentricity. The evolution of Equation 1.2 and Equation 1.3 can be visualized in the decay of the Hulse-Taylor binary's orbit (Figure 1.5). As the binary evolves with time, the semi-major axis and eccentricity of the orbit decay slowly over time due to the emission of GWs. As this system is essentially in an isolated environment, it will eventually be detected with marginal eccentricity $(e<0.1)$. 


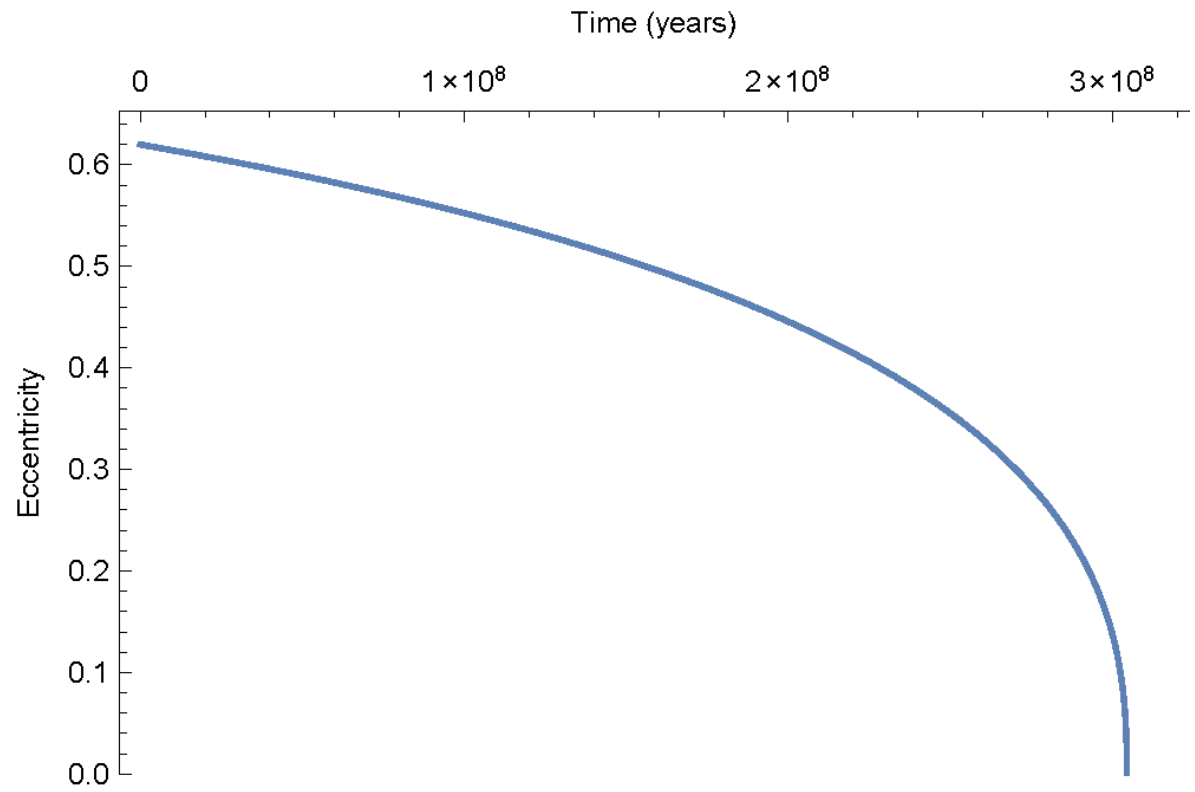

(a)

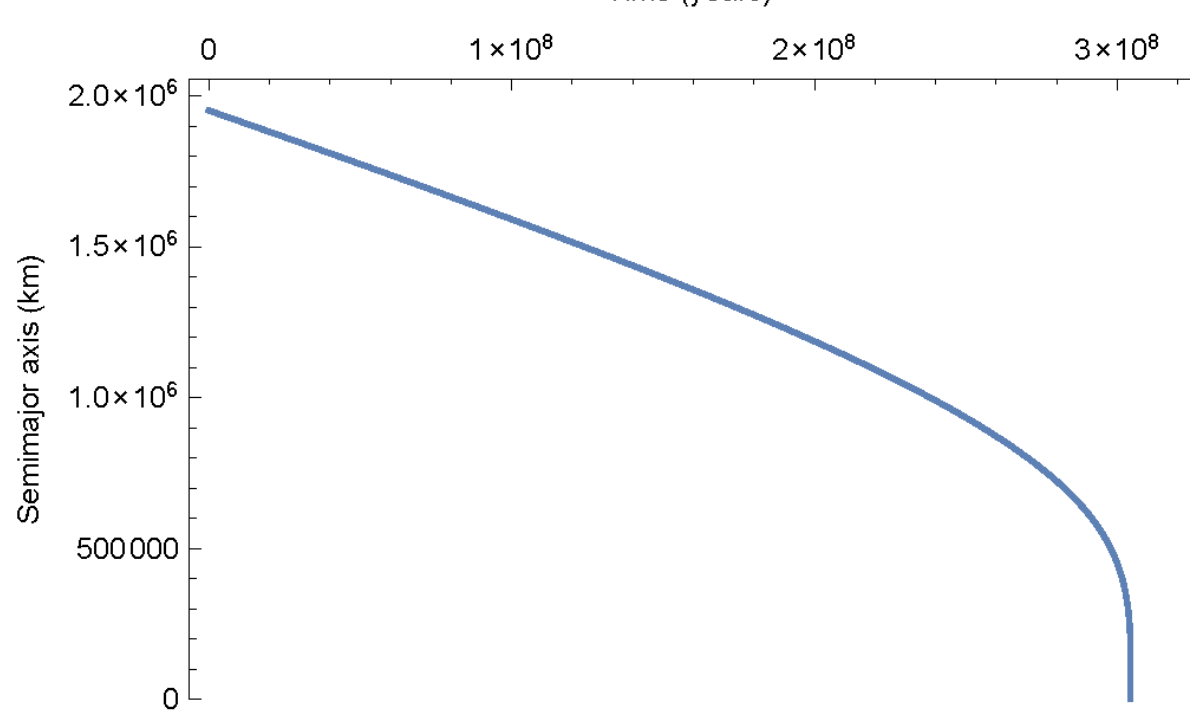

(b)

Figure 1.5: Figures showing the decay of the semi-major axis and eccentricity for the Hulse-Taylor binary as a function of time (years). Figure credit: Duncan A. Brown and Amber K. Lenon. top: Figure depicting the decay in the eccentricity of the orbit of the Hulse-Taylor binary as a function of time (years). bottom: Figure depicting the decay in the semi-major axis of the orbit of the Hulse-Taylor binary as a function of time (years).

Unlike circular binaries, where the GW emission is monochromatic and steadily 
increases in frequency and amplitude over time, eccentric GWs can be considered more "burst-like" in nature. This means they can, for instance, emit high-frequency GWs at closest approach, and low-frequency GWs as they move away from each other. This makes sense when one considers the motion of particle in an eccentric orbit dictated by Kepler's Laws. As a particle approaches periapsis, its velocity increases and as it approaches apoapsis its velocity decreases. So for an eccentric binary, at the moment where the two masses are closest together they will undergo a greater acceleration than when they are furthest apart. This leads to greater emission of GWs at periapsis than at apoapsis and can cause "bursts" of heightened-amplitude emission to appear in the signal. This asymmetric emission of gravitational radiation results in the orbit of the binary to go from elliptical to circular. The difference between a circular and eccentric waveform over time can visualized in Figure 1.6.

Another property of eccentric GWs that differentiates them from their circular counterpart is the average power radiated over one period. The full expression for the power, averaged over one orbit, radiated by an inspiraling binary has been shown by Peters \& Mathews (1963) to be given by

$$
\langle P\rangle=\frac{32}{5} \frac{G^{4}}{c^{5}} \frac{m_{1}^{2} m_{2}^{2}\left(m_{1}+m_{2}\right)}{a^{5}\left(1-e^{2}\right)^{7 / 2}}\left(1+\frac{73}{24} e^{2}+\frac{37}{96} e^{4}\right)
$$

where $G$ is the gravitational constant and c is the speed of light.

Note that this can be rewritten to explicitly separate the eccentricity compo- 
nents, where

$$
F(e)=\frac{1+\frac{73}{24} e^{2}+\frac{37}{96} e^{4}}{\left(1-e^{2}\right)^{7 / 2}}
$$

serves as an "enhancement factor", meaning the power radiated is significantly higher in objects with higher eccentricities. By setting $e=0$, the enhancement factor $F(e)$ has a value of 1 , and the expression simplifies to show the average power radiated by a circular binary given by

$$
\langle P\rangle=\frac{32 G^{4}}{5 c^{5}} \frac{M^{3} \mu^{2}}{a^{5}}
$$

where , $M$, the total mass, is given by $M=m_{1}+m_{2}$ and $\mu$, the reduced mass, is given by $\mu=\frac{m_{1} m_{2}}{m_{1}+m_{2}}$.

It is clear from Equation 1.4 that eccentricity serves only to enhance the power radiated from an inspiraling binary. However, an important influence that eccentricity has on gravitational radiation emission is the way it redistributes the power into different frequency harmonics. For a circular binary, the majority of the power is radiated in the second harmonic $(n=2)$ which leads to the relationship between GW frequency, $f_{G W}$, and orbital frequency, $f_{\text {orb }}$ as $f_{G W}=2 f_{\text {orb }}$. For higher eccentricities the relative power is actually spread out over multiple harmonics as seen in Figure 1.7 .

In Section 2.2, we will detail the information one can get out of observing binary black holes. To close this section, however, we will note in brief that because eccentricity has such a large impact on the induced strain as a function of time, by 

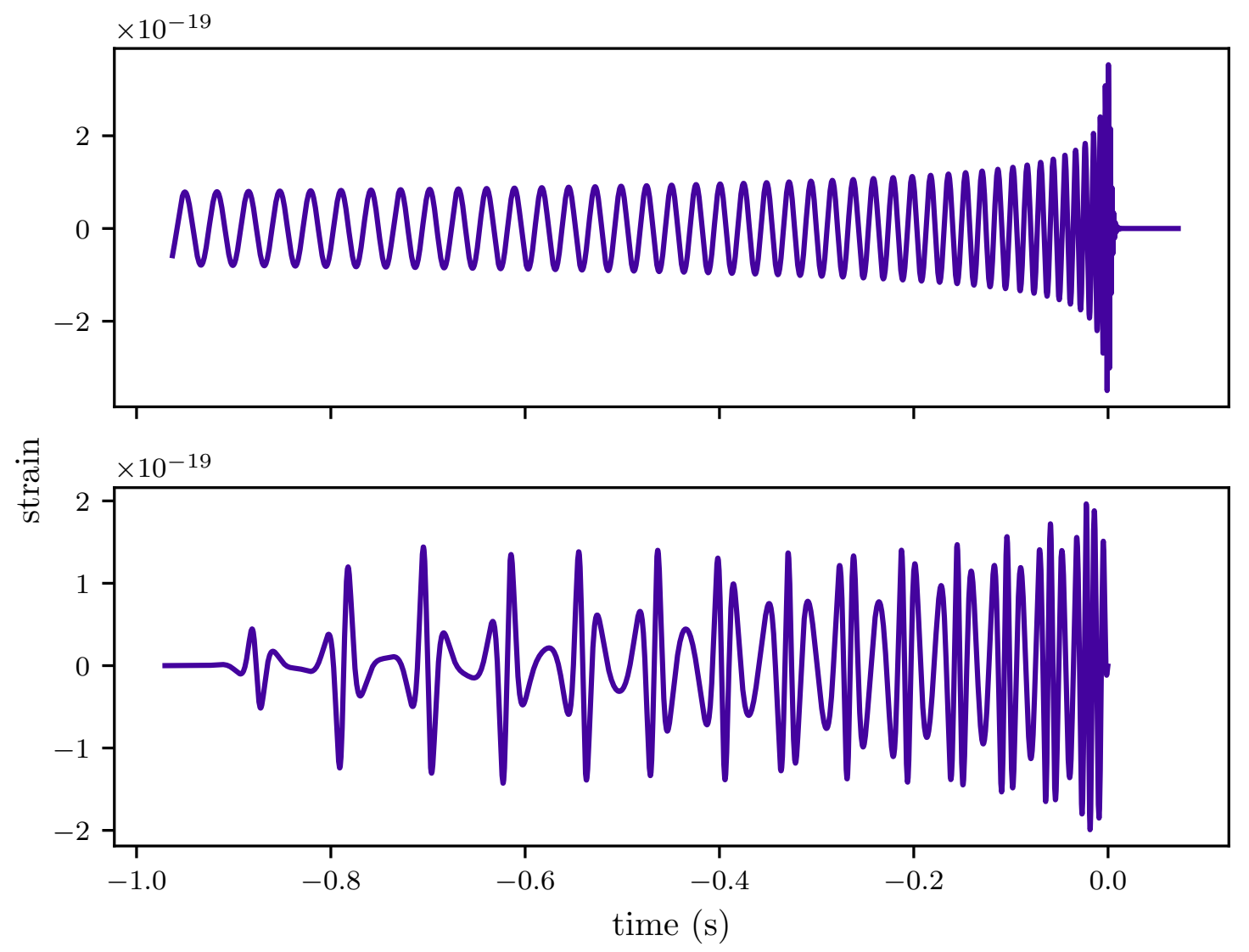

Figure 1.6: The difference in signals from a circular source vs an eccentric source. top: A GW signal generated by a circular source with $M=30 M_{\odot}, q=1$, and $f_{r e f}=30 \mathrm{~Hz}$. Where $q$ is the mass ratio and defined in this context as $q=m_{2} / m_{1}$. This signal was generated using waveform code EOBNRv2. bottom: A GW signal generated by an eccentric source with $M=30 M_{\odot}, e_{0}=0.3, q=1$, and $f_{\text {ref }}=30 \mathrm{~Hz}$. This signal was generated using waveform code EccentricFD (Huerta et al., 2014) 


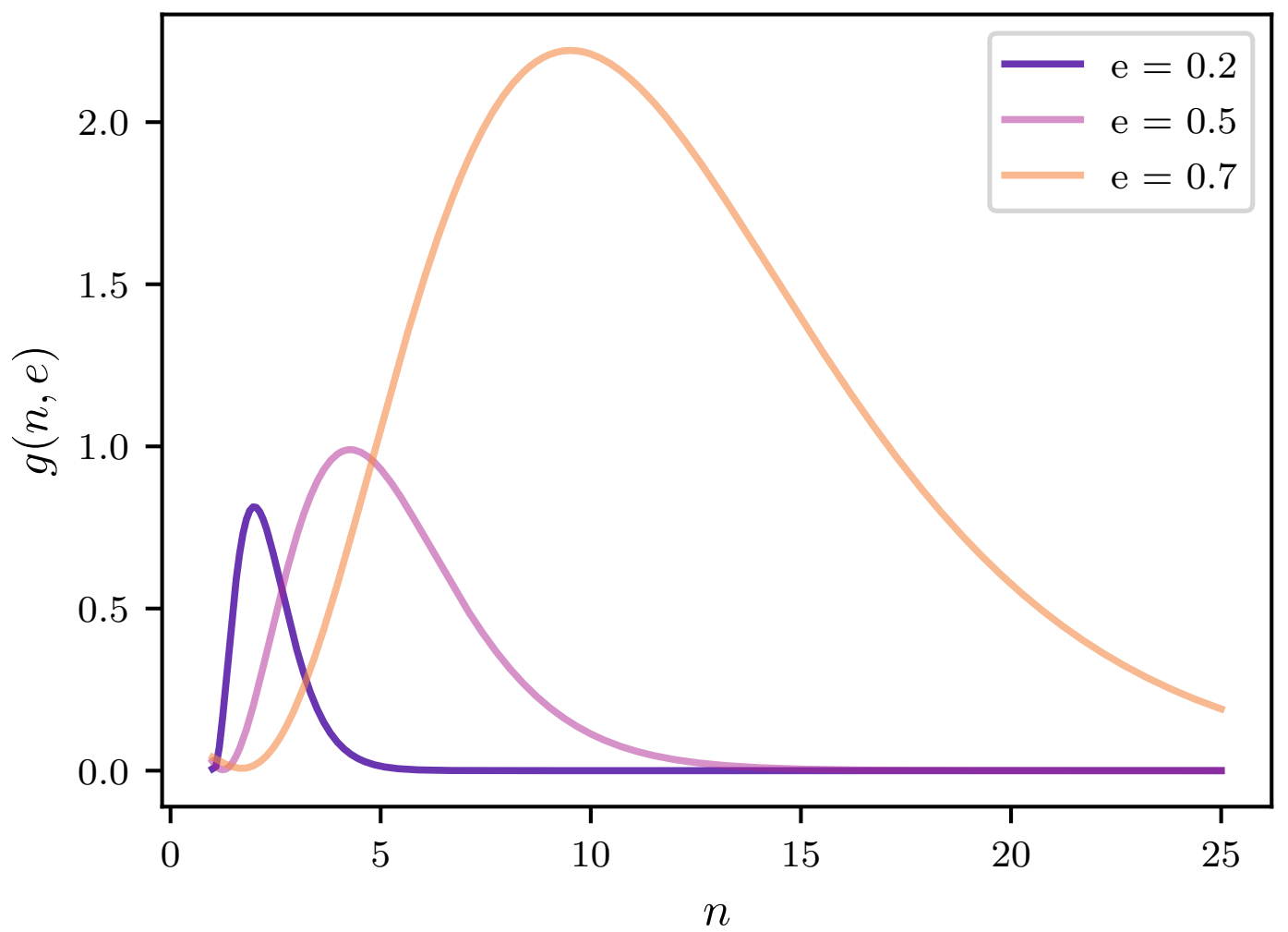

Figure 1.7: The relative power for specific eccentricities over different frequency harmonics. This is plot was generated using Equation 20 from Peters \& Mathews (1963). 
observing GWs from a BBH source, we can infer its eccentricity. This gives us a powerful probe of binary environments on both stellar- and super-massive scales. For instance, using the eccentricity of the binary, we can possibly determine the fraction of stellar-mass binaries that might have formed in either an isolated or dynamic environment (Abbott et al., 2019b).

Looking back at Section 1.1, binaries that merge due to the common-phase envelope will produce detectable GWs (García et al., 2021; Olejak et al., 2021). As this is an isolated environment, the binary will steadily lose eccentricity due to the emission of GWs and are expected to be detected with $e \leq 10^{-4}$ (Peters \& Mathews, 1963). A recovered eccentricity of $e>0.1$ could indicate instead that the binary formed in a dynamic environment rather than an isolated environment. For instance, GW captures could produce $e>0.1$ through three or four-body interactions with stars or compact objects (Rodriguez et al., 2018).

In the case of SMBHBs, there are several proposed mechanisms to solve the "last parsec problem": 1) dynamical friction through interactions with stars the stellar bulge (Mikkola \& Valtonen, 1992; Quinlan, 1996; Sesana et al., 2006), 2) interactions between the SMBHB and the circumbinary disk (Ivanov et al., 1999; Haiman et al., 2009; Kocsis \& Sesana, 2011), and 3) eccentricity, which hastens the rate of evolution of the binary (Peters \& Mathews, 1963; Peters, 1964) but can also be amplified by the first two solutions (Sesana et al., 2006, 2011; Sesana, 2010; Roedig \& Sesana, 2012; Armitage \& Natarajan, 2005; Cuadra et al., 2009; Roedig et al., 2011).

Each of these solutions could have some effect on the binary and cause the 
resultant GW signal to contain some non-negligible eccentricity. In some cases, for SMBHBs at the widest orbits, the binary may still be interacting with its environment, thus its orbital evolution may be driven by both GWs and environmental interaction. As with stellar-mass binaries, the recovered eccentricity of a SMBHB, and any detectable evolution in its orbit, could provide detailed information about its environment (Taylor et al., 2016) and further our understanding of galaxy evolution (Burke-Spolaor et al., 2019). 


\section{Chapter 2}

\section{Detection of GWs}

In Chapter 1 we reviewed the current understanding of the formation of $\mathrm{BBH}$ and SMBHB systems and GWs. We also looked at how detecting eccentric GWs can improve our understanding of these systems by constraining from which environment they formed. In this chapter we will look into two types of GW detectors, groundbased and PTA, and examine the motivation/challenges of searching for eccentric GW sources in these regimes.

\subsection{GW Detectors}

As GWs propagate away from an emitting system, they cause the surrounding space-time to become stretched and squeezed. The amount of space-time distortion due to the propagation of GWs is called strain. Strain is generally defined (in materials science and GW science) as a fractional change in length if one considers two points in space-time separated by some distance $L$. If a GW is propagating through this space-time, then it would change the distance between the points by some amount $\Delta L$. Therefore, the fraction of these two quantities will give information about the strain amplitude of the propagating GW which can be described as

$$
h=\frac{\Delta L}{L} .
$$


Different sources emit GWs at various strains and frequencies resulting in various detectors that are needed to probe the wide range of frequencies that make up the GW spectrum (Figure 2.1).

Looking at Equation 2.1, it can be inferred that to be sensitive to smaller changes in length, one would need a detector with longer baselines. Of course, these longer baselines come with greater physical/astrophysical challenges, but this is necessary to probe the range of frequencies generated by various GW sources. In this section we will mainly focus on ground-based interferometers and pulsar timing arrays as detectors of GWs.

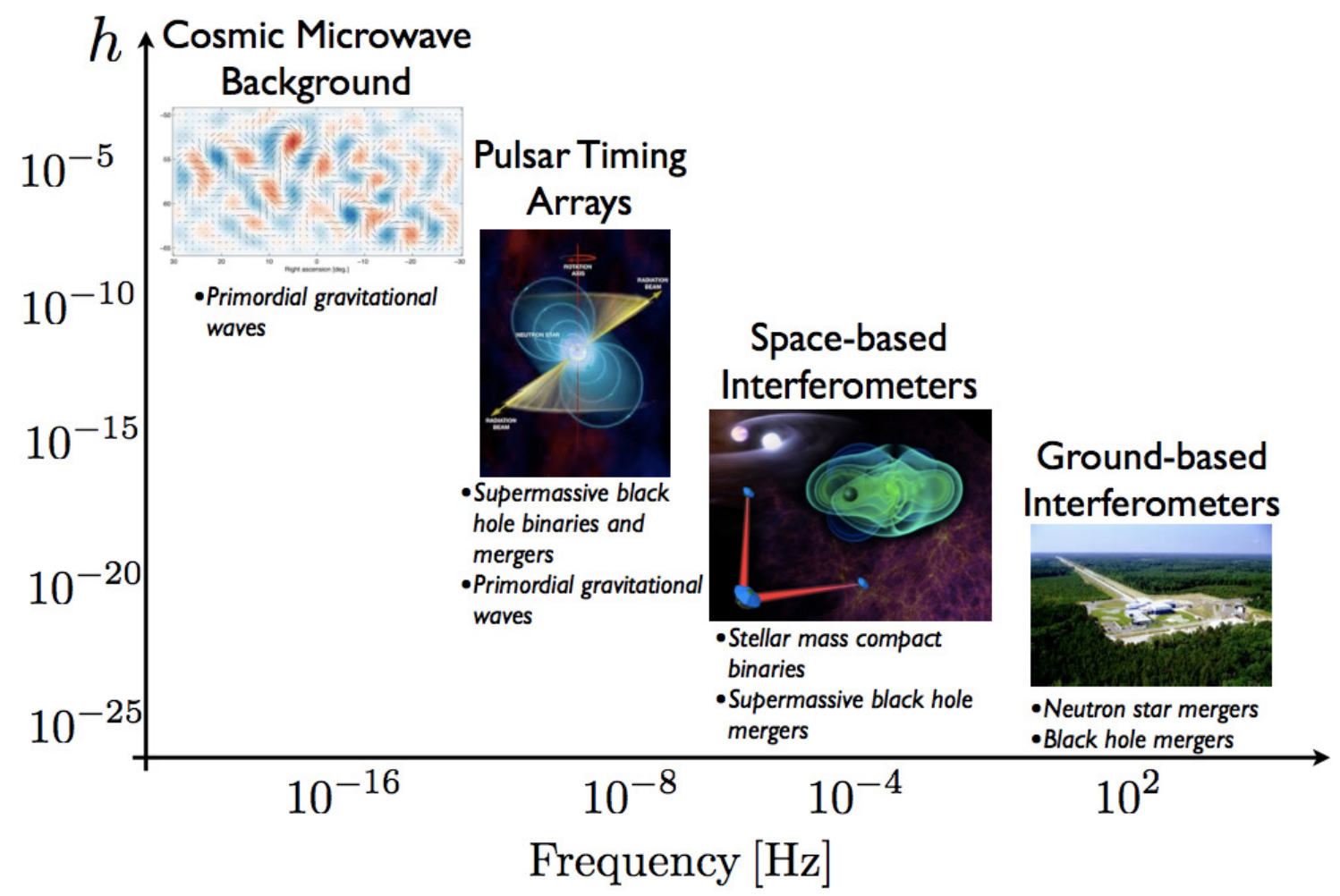

Figure 2.1: GW spectrum with types of detectors for different frequency bands and sources to which each of these detectors are sensitive. Image Credit: NANOGrav 


\subsubsection{Ground-Based Detectors}

In the 1960s, an effort was made by Joseph Weber, a physicist, to develop a detector to detect the GWs predicted by Einstein more than 40 years prior. The first detector he developed used two large aluminum cylinders to measure the vibrations of a passing GW (Caltech, 2016). The use of Michelson interferometery, which is described below, was first suggested by Michael Gertsenshtein and Vladislav Pustovoit, and also independently by Joseph Weber and Rainer Weiss (Caltech, 2016). This became the foundation of the LIGO that we know today.

In the modern era of GW science, the Laser Interferometer GW Observatory (LIGO, Aasi et al. 2015), Virgo (Acernese et al., 2015), and the Kamioka GW Observatory (KAGRA, (Aso et al., 2013)) is the culmination of decades of work to develop ground-based GW detection instruments. LIGO consists of two detectors with one located in Hanford, Washington and another in Livingston, Louisiana. Each detector has two arms $4 \mathrm{~km}$ in length that house a system of lasers and mirrors encased in vacuum-sealed tunnels. This complex system of lasers and mirrors is setup to use Fabry-Perot Michelson interferometry to measure the change in path length of the laser light as a GW passes through Earth. A simplified version of a Michelson interferometer - can be seen in Figure 2.2. The GW-induced change in path length is measured as a phase difference between the two laser beams over a certain period of time. This phase difference can be caused by any perceived change in path-length, including vibrations of the instrument or mirror, but importantly, can be induced by the strain in space-time produced by a propagating GW (Aasi 
et al., 2015).

To be sensitive to the tiny ripples in space-time, one must account for the other possible sources of noise for a ground-based detector like aLIGO. In Figure 2.3 approximations to the noise curves for aLIGO (Advanced LIGO), Virgo, and KAGRA are shown along with the region in which compact binary inspirals are expected to occur in this part of the GW spectrum. Looking at the aLIGO noise curve, its overall shape is close to log-quadratic, and there are two prominent noise "spikes."

The big spike $(\sim 10 \mathrm{~Hz})$ comes from the combination of seismic noise from the Earth and noise from the suspension system that holds the mirrors in place. The smaller spike $(\sim 500 \mathrm{~Hz})$ also occurs because of the violin modes. At low frequencies $(f<10 \mathrm{~Hz})$, the curve is dominated by environmental noise (e.g. seismic motion and acoustic thermal noise) and at higher frequencies $(f>100 \mathrm{~Hz})$ the curve is dominated by quantum noise (e.g. input laser power). All of the major aLIGO noise sources, like the violin modes of the wires holding the mirrors to the quantum noise, have been well-documented and budgeted into the detector's design since its conception (Abbott et al., 2009).

In addition to the sources described above, there are plenty of other noise transients (i.e. glitches) that can either induce a signal in these detectors or dominate the data. For example, the first binary neutron star detection, GW170817, was dominated by a loud noise transient (Abbott et al., 2017a). If a noise transient dominates a potential signal, they can be removed before an analysis is conducted. Some noise transients could be confused for an actual GW signal, but a multidetector network allows us to check the validity of any potential signals. 


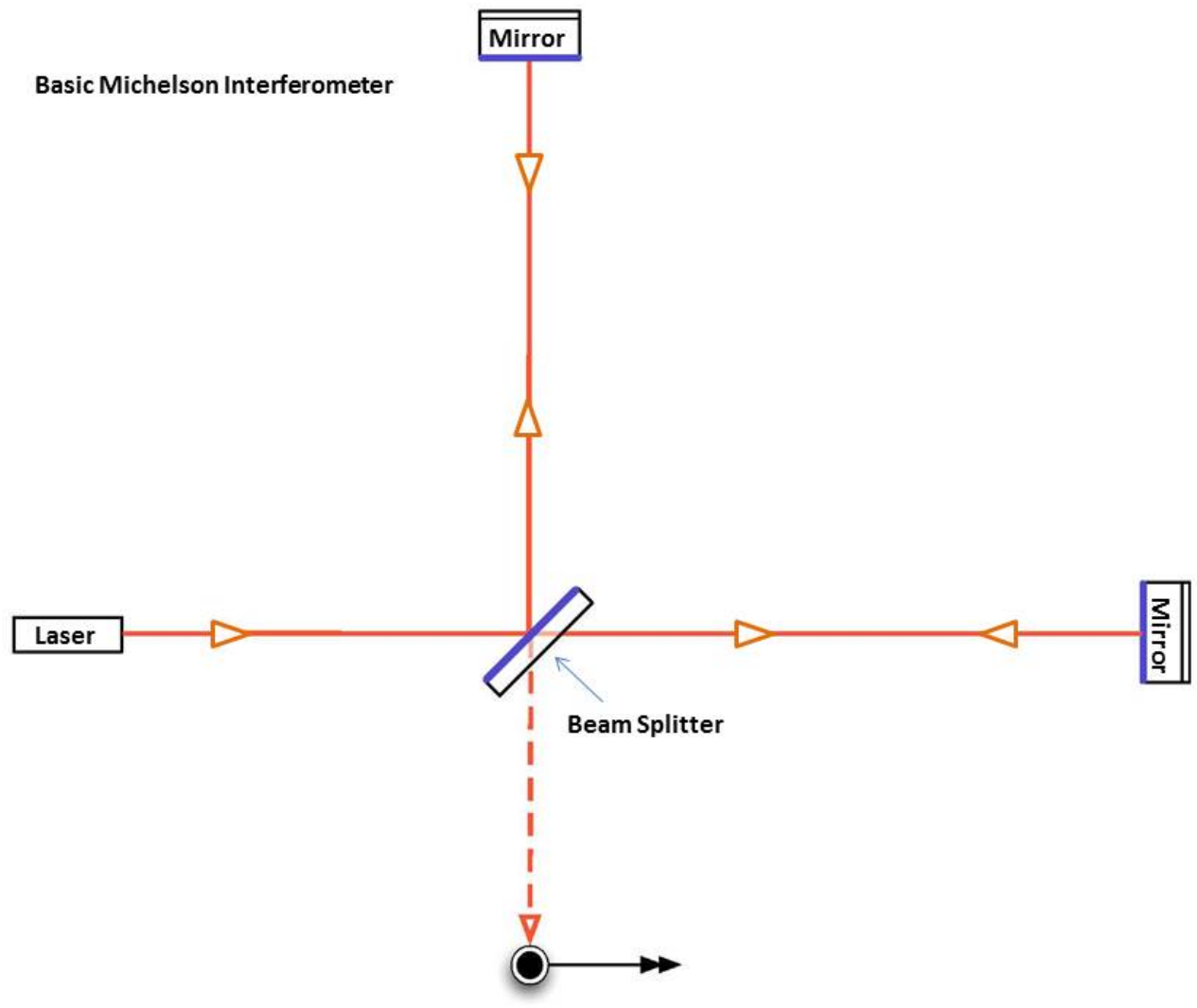

Figure 2.2: This diagram shows the basic setup of a Michelson interferometer. On the left is a laser sending light through the beam splitter. The beam splitter sends the light down two separate paths that each contain a mirror at the end. The laser light is reflected back towards the beam splitter where it is combined and detected by a photo diode to check the phase of the light. If the combined light is out of phase then they will destructively interfere with each other, but if they are in phase then they will constructively interfere with each other. When a GW passes through the detector it causes the path lengths of each arm to either be compressed or stretched which affects the phase of the combined light detected by the photo diode. Image credit: LIGO 


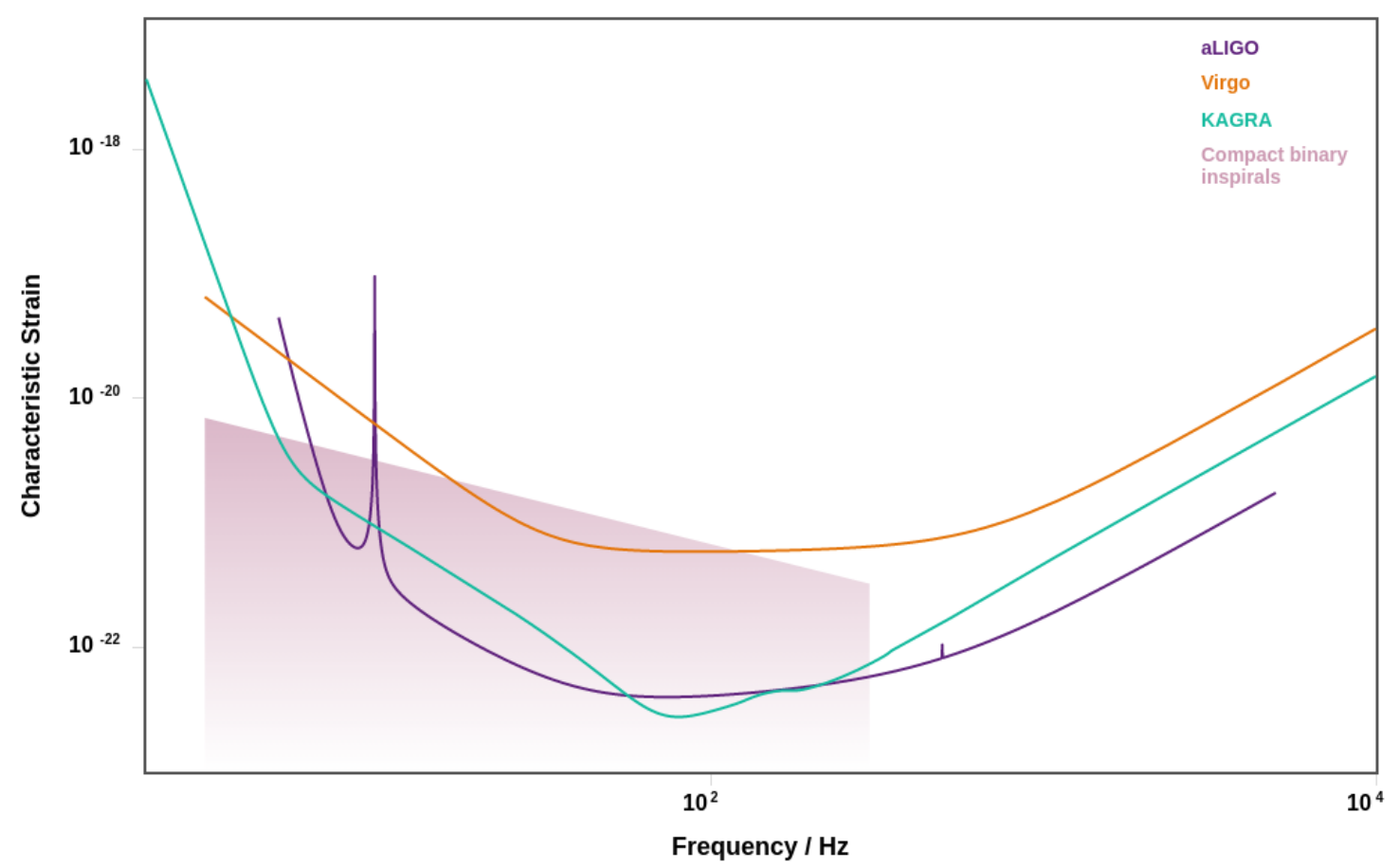

Figure 2.3: Sensitivity curves for the active ground-based GW detectors. The yaxis shows the characteristic strain, which is a convention used for determining the signal-to-noise ratio of a source, but is not representative of a physical signal from an individual GW source. This figure was created using a resource developed by Moore et al. (2015). 


\subsubsection{Search Methods}

When it comes to searching over GW data there are several parameters that are important to constrain a $\mathrm{BBH}$ system (i.e. $M$, q, etc.), but one of the important parameters is its chirp mass,

$$
\mathcal{M}=\left[\frac{q}{(1+q)^{2}}\right]^{3 / 5} M=\frac{c^{3}}{G}\left(\frac{5}{96} \pi^{-8 / 3} f^{-11 / 3} \dot{f}\right)^{3 / 5}
$$

where $f$ is the GW frequency and $q=\frac{m_{2}}{m_{1}}$ assuming $\frac{m_{2}}{m_{1}}<1 . \mathcal{M}$ encodes the

frequency evolution, $\dot{f}$, making it covariant with $\dot{f}$. From the GW data it is possible to obtain $\mathcal{M}$ and $M$ from which it's possible to calculate $q$ and constrain the other parameters of the BBH system.

In the case of searching over ground-based GW data, there are two methods that are typically employed: matched-filtering and burst searches.

Matched-Filtering (Cannon et al., 2012; Usman et al., 2016; Allen et al., 2012) takes a filter (in this case, a waveform of a specific shape) and essentially sifts through the data until it finds a matching signal. In aLIGO, these filters are known as template banks, and are generated from waveform codes that use numerical relativity techniques and/or expansions on Newtonian gravity to model the GW emission from a binary, given its orbital parameters. Template banks are a set of templates designed to search over the whole binary orbital parameter space (i.e. primary $\left(m_{1}\right)$, secondary $\left(m_{2}\right)$ masses, etc.). To cover the whole search parameter space, the template banks have to contain a large number of templates with some searches 
having on order millions of templates. To calculate the correlation between a possible signal in the aLIGO and Virgo dataset with a particular waveform template, the noise-weighted inner product between the signal and the template is calculated. The equation is as follows (Abbott et al., 2016b):

$$
\langle s \mid h\rangle(t)=4 \int_{0}^{\infty} \frac{\hat{s}(f) \hat{h}^{*}(f)}{S_{n}(f)} e^{i 2 \pi f t} d f
$$

where $s(t)$ is the signal strain, $h(t)$ is the waveform template, $S_{n}(f)$ is the power spectral density of the noise, $\hat{s}(f)$ and $\hat{h^{*}}(f)$ are the Fourier transformations of $s(t)$ and $h(t)$. Although the data is a function of time, this calculation is done in the Fourier domain because it is easier to characterize the noise in frequency (e.g. as in Figure 2.3). As an example of matched-filtering, this technique is how each of the sources shown in Figure 2.5 were detected; they used template banks generated from waveform codes that model circular binary systems.

Burst Search Methods like BayesWave (Cornish \& Littenberg, 2015; Littenberg \& Cornish, 2015; Cornish et al., 2021) and Coherent Wave Burst (cWB, Klimenko et al. 2008) are a type of unmodeled approach to searching for signals in GW data. For the sake of the work discussed in Chapter 3, this part of the discussion will focus on BayesWave. The "Bayes" in BayesWave represents the Bayesian inference used by the method to discern what type of signal is in the data. This is done by first determining what is known as the posterior distribution. The posterior distribution is the probability that a GW signal, $\mathbf{h}$, as described by the model, $M$, is present in 
the GW data, s. The posterior can be calculated as (Cornish \& Littenberg, 2015)

$$
p(h \mid s, M)=\frac{p(h \mid M) p(s \mid h, M)}{p(s \mid M)},
$$

where $p(h \mid M)$ is the prior probability and represents the assumptions made by the model, $M$, about the GW signal, $h$. The likelihood, $p(s \mid h, M)$, is the probability of the GW data, $s$, containing $\mathbf{h}$ given $M$. Finally, the Bayesian evidence or marginalized likelihood, $p(s \mid M)$, is the probability of the GW data, s, containing a signal given any model, $M$.

BayesWave calculates the posterior distribution using various forms of the Markov Chain Monte Carlo (MCMC) method. MCMCs are statistical methods that can be used to randomly sample multi-dimensional probability distributions. In combination with the prior distribution, BayesWave calculates Bayes factors that can be used to discern the nature of a signal detected in the GW data. Which means that this signal is either purely noise or astrophysical in origin. A visual example of how Bayes factors of different models are compared can be seen in Figure 2.4. This figure shows three regions that represent three different models that could be present in the data: just noise, noise and a GW signal, or noise and a glitch, where a glitch is a noise artifact that is not astrophysical in nature. The Bayes factors calculated for a given analysis determines which model best applies to the data. In the case of Figure 2.4, BayesWave determined that a signal was present in the simulated GW data (this is represented by the "+" symbol next to "Signal").

The "wave" part of BayesWave refers to its use of wavelets to reconstruct 


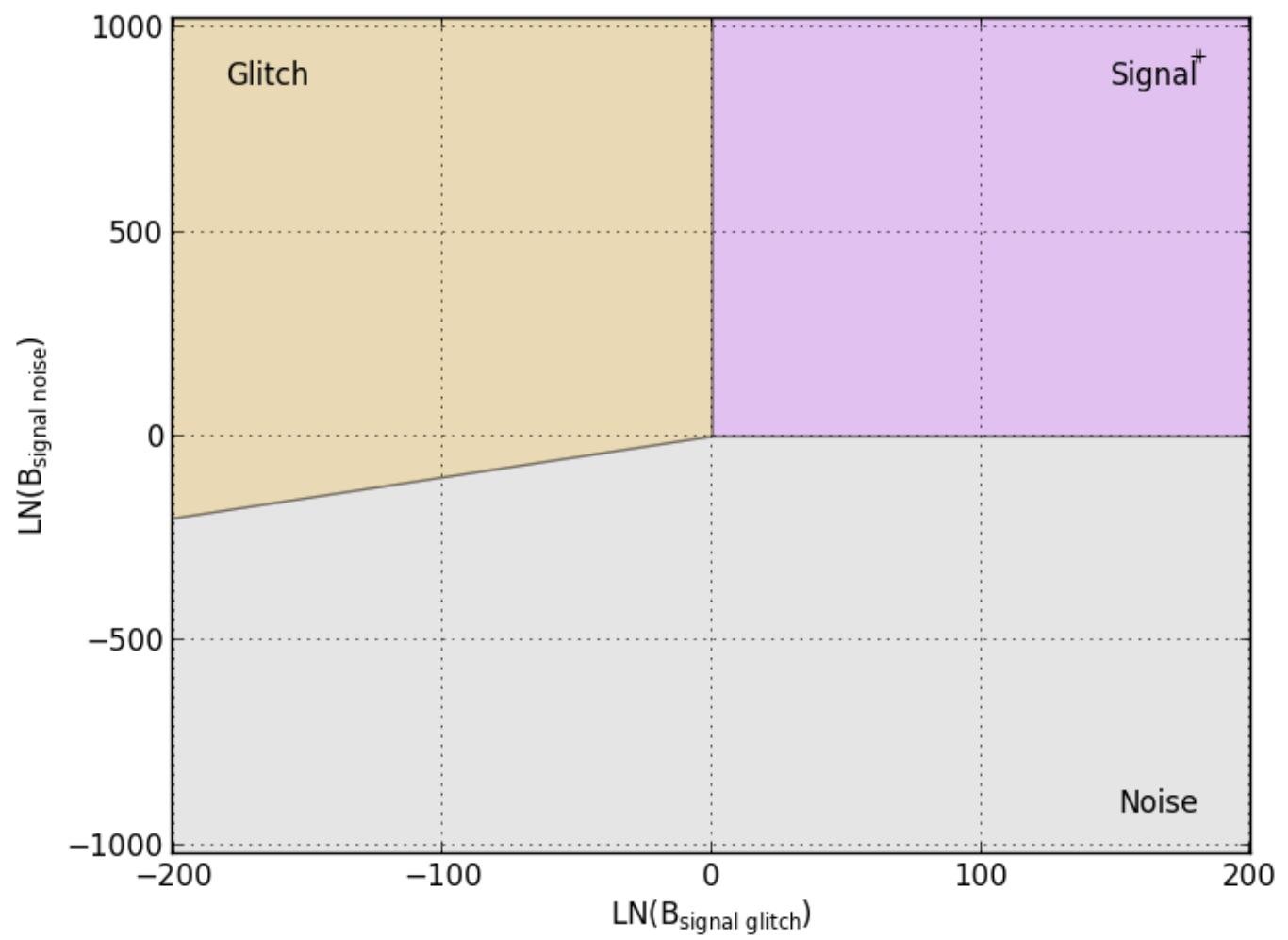

Figure 2.4: This plot compares the natural logarithm of the Bayes factor for a signal model compared to a noise-only model, $\mathrm{LN}\left(\mathrm{B}_{\text {signal noise }}\right)$, and the natural logarithm of the Bayes factor for a signal model compared to a glitch model, $\mathrm{LN}\left(\mathrm{B}_{\text {signal glitch }}\right)$. Each region on the plot marks the type of model and any point in any of these regions marks which model was preferred. For this case, the signal model was preferred and this is indicated by the "+" symbol above "Signal". This plot was generated using BayesWave (Cornish \& Littenberg, 2015; Littenberg \& Cornish, 2015; Cornish et al., 2021). 
a signal that may be present in the data. By nature, wavelets are well-localized in time and frequency and are suitable for sifting out excess power in GW data. Using wavelets as a template to piece together parts of a signal is what makes this method model-independent but less sensitive than model-based methods like matched-filtering.

\subsubsection{The Status of Detections by Ground-based Detectors}

Ground-based detectors like Virgo, KAGRA and the future Indian GW Observatory (IndiGO, Unnikrishnan 2013) are conceptually based on the LIGO detectors and all form a vast ground-based detector network. This network is sensitive to compact binary inspirals and mergers from binary neutron stars, neutron star black hole binaries, and binary black holes. As previously noted, with two detectors one can confirm the existence of a signal, however with three or more we gain better localization of a detected cosmological source. Localization, in turn, is critical for permitting multi-messenger studies, as it gives a smaller area for follow-up searches with telescopes operating on the electromagnetic spectrum.

Detections by aLIGO/Virgo have been found using both methods described in Section 2.1.1.1. A visual summary of the detection spectrograms (GW-frequencydependent power as a function of time) from aLIGO and Advanced Virgo's observing period of 2015 - 2019 is shown in Figure 2.5. In the first and second observing runs,

10 BBHs were detected as well as the first BNS merger (Abbott et al., 2019a, 2017b). The BNS merger has provided insight into the mystery of kilonovae (now conclusively 
shown to be transient events produced by the collision of two neutron stars) and improved constraints on the equation of state for the core of a neutron star (Abbott et al., 2017c). The third observing run added an additional 39 more detections, with 36 of those being from BBHs, an additional BNS merger, and the first black hole compact object merger, where the compact object could be the lowest mass black hole or highest mass neutron star ever (Abbott et al., 2020, 2021a). Further analysis has brought the total count of GW detections to 57 (Nitz et al., 2020, 2021). Recently, two neutron star black hole binaries were detected (Abbott et al., 2021b). 


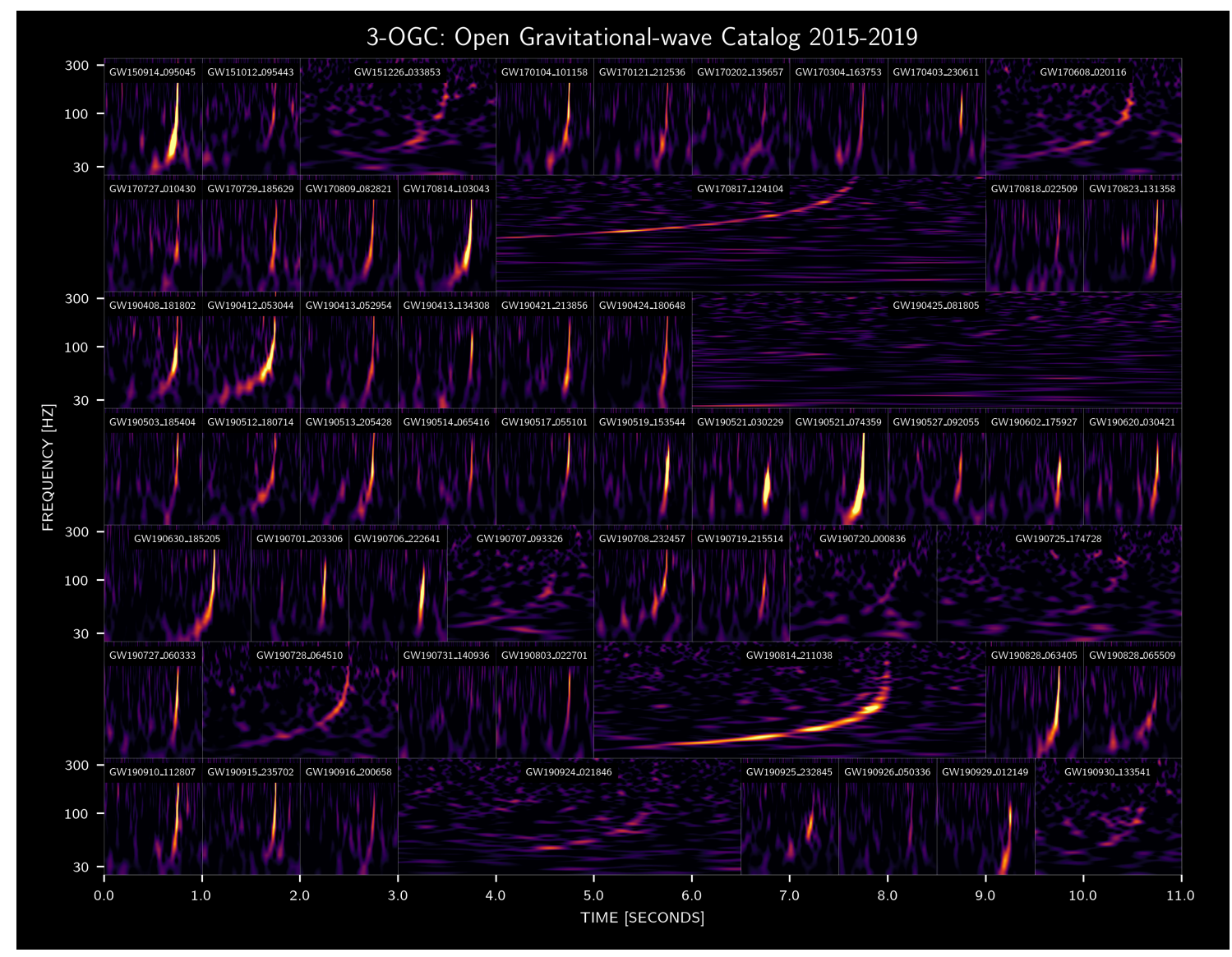

Figure 2.5: GW transients from the open GW catalog of aLIGO and Virgo observing runs during 2015-2019. These are shown in time-frequency space. For each source, the time-frequency track is shown where the brightest part of the signal is called the "chirp," which tracks the binary through its evolving orbit from wide separations (low frequency) to small separations (high frequency). Image Credit: Nitz et al. (2021) 


\subsubsection{Pulsar Timing Arrays}

The first pulsar was discovered in 1967 by Jocelyn Bell-Burnell (née Bell) as she was looking at radio data from the Interplanetary Scintillation Array and noticed a periodic pulse of radio emission in the data (Hewish et al., 1968). More observations of these strange sources revealed that pulsars are a type of neutron star that generates a beam-like radio emission from its magnetic poles. As the pulsar rotates and its beam passes by our line-of-sight, a radio pulse is observed. A diverse range of pulsar phenomenologies are often discussed, and are typically characterized by their period, period derivative, magnetic field, and emission characteristics (Lorimer \& Kramer, 2012).

For GW detection, the most important phenomenological class of pulsars is "millisecond pulsars" (MSPs), which refers to pulsars that have been spun-up by interaction with a companion such that they are rotating hundreds of times per second, thus with millisecond-duration periods. The first MSP was detected by Backer et al. (1982). Long-term observations of MSPs have revealed that they have very precise periods (Davis et al., 1985). After the first extended observations of MSPs, it was postulated by Foster \& Backer (1990) that by timing many MSPs in a coordinated array, one can possibly observe a background of GWs. The GW background (from any cosmic source) will induce a correlation of the pulsar timing residuals shown by the Hellings and Downs curve (Hellings \& Downs, 1983). In the presence of an isotropic, unpolarized GW stochastic (random) background, where GWs obey the characteristics predicted by General Relativity, the Hellings and 
Downs curve will look similar to Figure 2.6. The curve shown in Figure 2.6 is expected for GW sources with the two polarization modes (plus and cross, see Section 1.2) predicted by GR. If there are other polarization modes present (due to deviations from GR) or if the background is not isotropic, then the Hellings and Downs curve will look different (Jenet \& Romano, 2015).

MSPs tend to have very stable rotational periods over a long time, making them suitable to time to the sub-microsecond precision necessary for detecting GWs (Jenet et al., 2006). The use of MSPs for pulsar timing arrays (PTAs) is a staple of the experiments being carried out to this day. PTAs have multiple MSPs and the distance between the earth and an MSP in the array can be thought of as one baseline. Having multiple baselines is similar to why there are multiple ground-based detectors in the ground-based detector network. It helps to confirm the presence of a GW signal and locate its source on the sky. As a result of these long baselines, current PTAs are sensitive to GWs in the frequency range of $10^{-9}<f_{G W}<10^{-6} \mathrm{~Hz}$ and are expected to detect the GW background and individual, continuous-wave sources of GWs coming from SMBHBs.

There are several world-wide efforts whose primary aim is PTA science. The International Pulsar Timing Array (IPTA) is made up of the North American Nanohertz Observatory for GWs (NANOGrav), the European PTA (EPTA), the Parkes PTA (PPTA), the Indian PTA (InPTA), and has ongoing growth as it expands to other emerging PTAs worldwide. This consortium of PTAs exists for the collaboration of scientists around the world in the sharing of data and data analysis techniques. Since each PTA has access to a limited number of telescopes, limited 


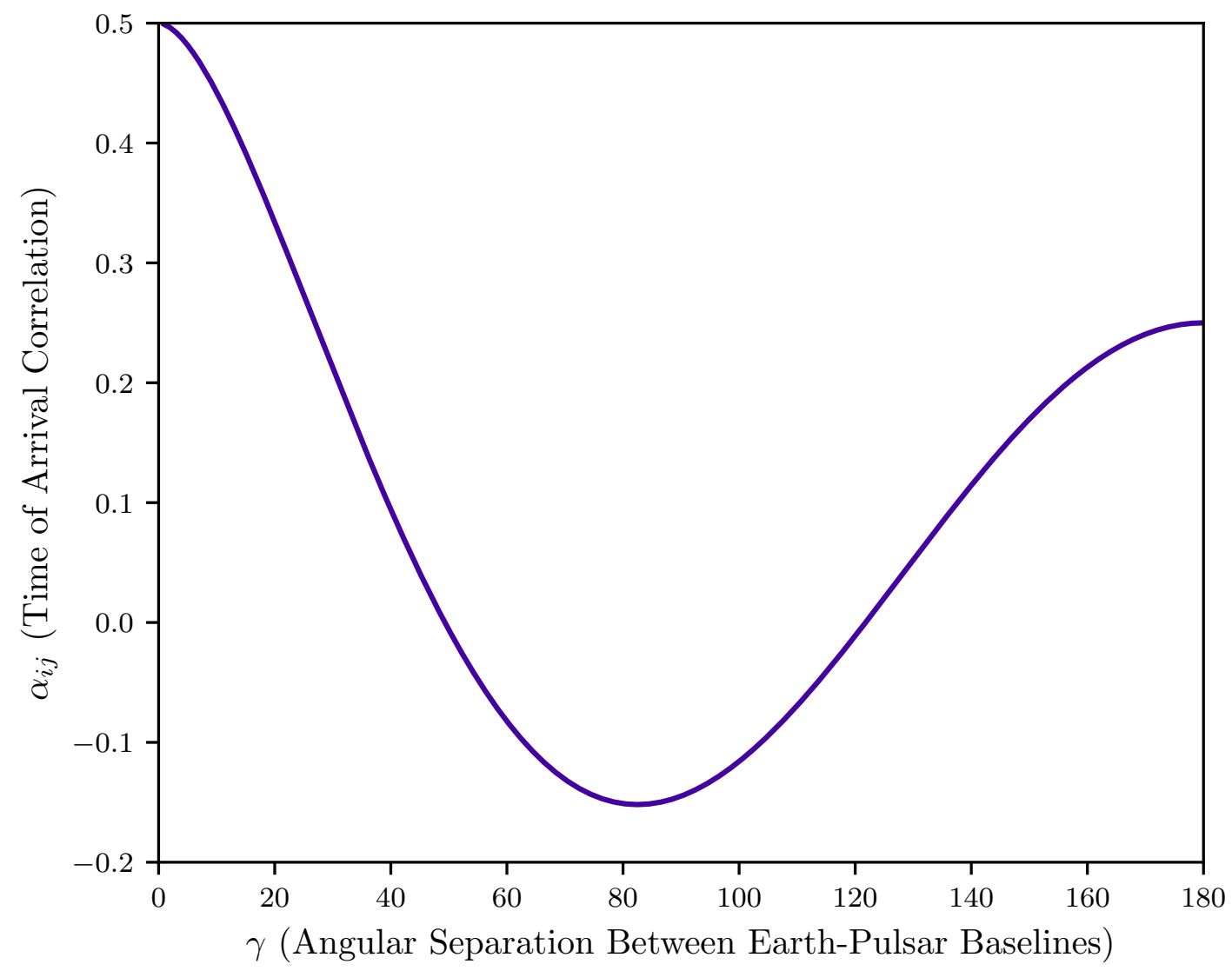

Figure 2.6: The Hellings \& Downs curve shows the correlation in pulsar times-ofarrival (y-axis) between a set of pulsars in a array (x-axis) in the presence of an isotropic, unpolarized GW stochastic background. $\alpha_{i j}=1 / 2$ when $\gamma=0$ comes from the fact that this correlation is present in the Earth term and not the Pulsar term and therefore less than half of the contribution should be accounted for at this particular point. This y-intercept changes depending on normalization. Plot generated using Equation 1 from Jenet \& Romano (2015) based on Equation 5 from Hellings \& Downs (1983) 
observation times, and (in the case of Northern/Southern Hemispheres) different pulsars, combining international data makes it easier to get more complete and sensitive timing data. Each PTA has a certain number of pulsars in its array, and most PTAs are always adding new pulsars, since surveys for well-timed MSPs are ongoing. For example, NANOGrav has 47 pulsars in their public 12.5 year dataset (Alam et al., 2021a,b). For the IPTA as a whole, the Second IPTA Data Release had more than 65 pulsars that are timed regularly by NANOGrav, the EPTA, and the PPTA (Perera et al., 2019).

\subsubsection{Pulsar Timing Data}

The process of pulsar timing produces timing residuals, which we use for our detection analysis. Here we aim to detail the process by which this data we use is obtained. A schematic of the process can be seen in 2.7. First, we start with the radio observations of a pulsar. The receiver on the radio telescope sends the observation data through a pipeline that de-disperses and folds the data. De-dispersion is a process that removes frequency-dependent delays in pulsar pulses, which are caused by dispersion in the interstellar medium.

The dispersion measure (DM) quantifies the delay, and is measured upon the initial discovery of the pulsar. DM is the measure of free electrons in the interstellar medium along a particular line-of-sight. The process of folding cuts the incoming data into smaller time segments that are the length of the pulsar's rotational period, averaging sequential phase-resolved pulses together to increase the signal-to-noise 


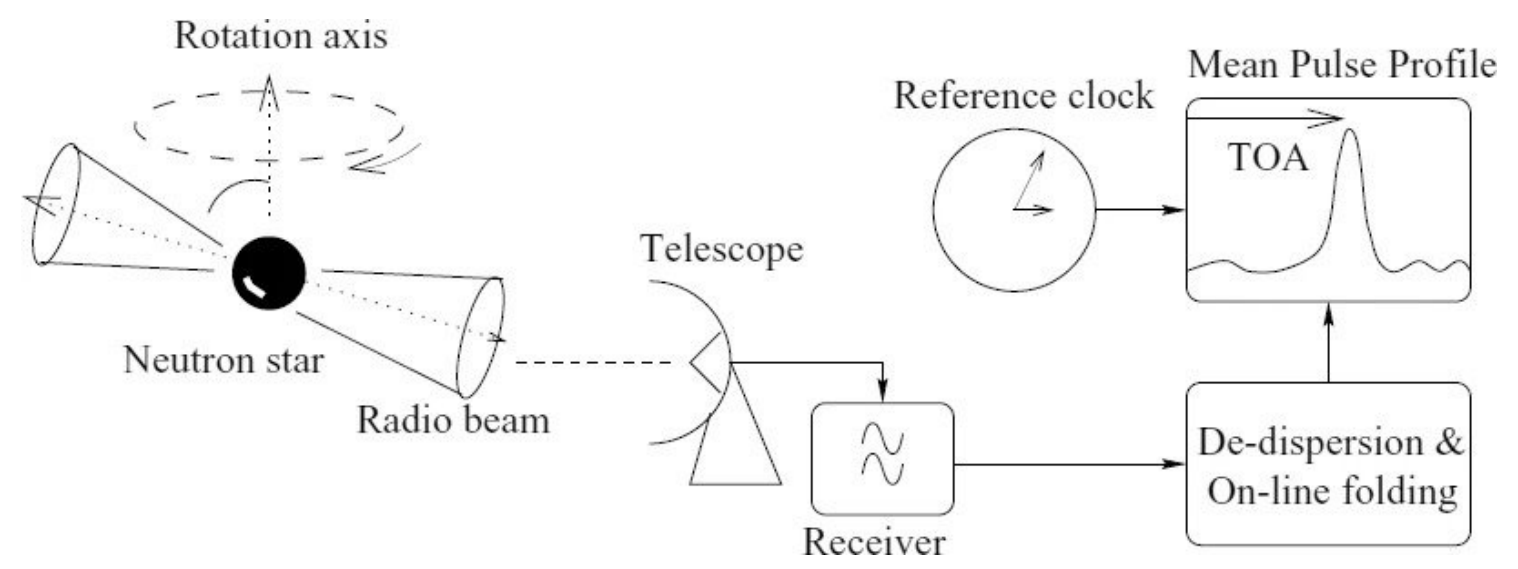

Figure 2.7: Schematic showing the process of timing a pulsar. Figure Credit: Lorimer (2001)

ratio (SNR) of the pulse. This effectively creates a distinct pulse shape called a pulse profile. This pulse profile is then compared to the profile template, a zeronoise modelled version of the pulse profile. The difference calculates the time of arrival (TOA) of that time-averaged pulse from the observed pulsar. One TOA is calculated from time chunks of $20 \mathrm{~min} \sim 1 \mathrm{hr}$ which contains information from many rotations of that pulsar.

Once we have a set of TOAs, to produce a GW-searchable data stream we must first compare the TOAs to a model of the pulsar, such that we can produce the fundamental search data product of PTAs: the timing residuals. A timing residual is the subtraction of the observed TOAs from the TOAs predicted by the timing model for each pulsar. The timing model includes measurements of the period, the period derivative, sky location, proper motion of each pulsar, in addition to potentially many other parameters measured from timing (for instance if the pulsar is in a binary system, the binary parameters must be accounted for).

Note that this only accounts for the motion of the pulsar, but the motion of 

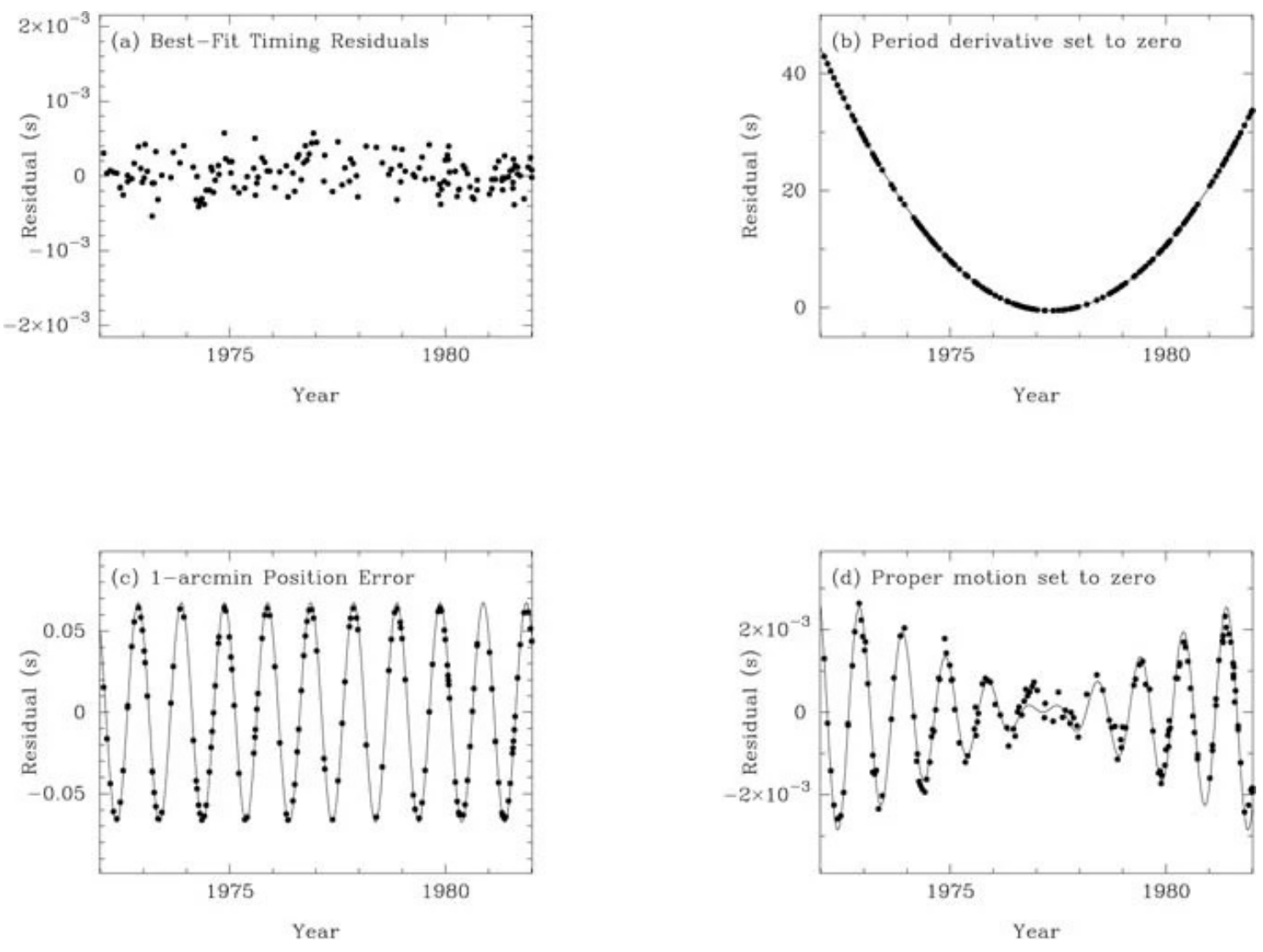

Figure 2.8: Figure showing the effect of different astrophysical errors on the timing residual of a pulsar. Panel a) shows a residual when the timing model fits the data accordingly; b) shows the effect of period derivative error; c) shows the effect of an error in the sky location of a pulsar; d) shows the effect of an error in the proper motion of the pulsar. Figure Credit: Lorimer (2001)

the Earth must be accounted for too. As the Earth is a member of the solar system and the solar system orbits the galaxy, TOAs are typically referenced to the the solar system barycentre using time-dependent dynamics that are calculated from the solar system ephemeris in tandem with Earth-based time measurements from atomic clocks.

If the timing model properly accounts for all major effects in the TOAs, and there are no or only weak GWs present, then it will produce a residual like panel (a) of Figure 2.8. If there is an error in the model's pulsar period derivative, then the 
resultant residual would look similar to panel (b) of Figure 2.8. If there is an error in the sky position of the pulsar, then the motion with respect to the solar system's barycenter will produce an annual cycle which would appear in the residuals as shown in panel (c) of Figure 2.8. Finally, if the proper motion of the pulsar is not accounted for, then it will produce a residual similar to panel (d) of Figure 2.8. Over time, pulsar parameters are built by observations of the pulsar and re-modelling of the residuals to obtain a better-fit pulsar model. More observations not only increase our understanding of these effects and helps to properly fit for these effects so that we don't attribute them to actual GWs.

There are other effects that can contribute to the noise that are intrinsic or extrinsic to each pulsar in a PTA. These effects can include time-varying DM, changes in pulse shape, jitter, or intrinsic neutron-star instabilities, etc. and can be classified into two types of noise: white (uncorrelated) noise and red (correlated) noise. Since these noise effects can affect the timing of pulsars and the potential detection of a GW signal, they are typically fit for when doing a GW search in PTA data. More detail on how white and red noise models are built in to a GW-signal search will be provided in Chapter 4 .

Figure 2.9 shows a simulated sensitivity curve based on NANOGrav's 12.5 year dataset. The shape of this curve is dependent on several different factors. The higher frequency end $\left(f>10^{-8}\right)$ depends on the timing precision of the constituent pulsars and their observation cadence. The big spike represents $1 / \mathrm{yr}$, the smaller peak next to it represents $2 /$ yr or 1 every 6 months, and smaller bumps after come from the most sensitive pulsars. The lower frequency part of the curve $\left(f<10^{-8}\right)$ 


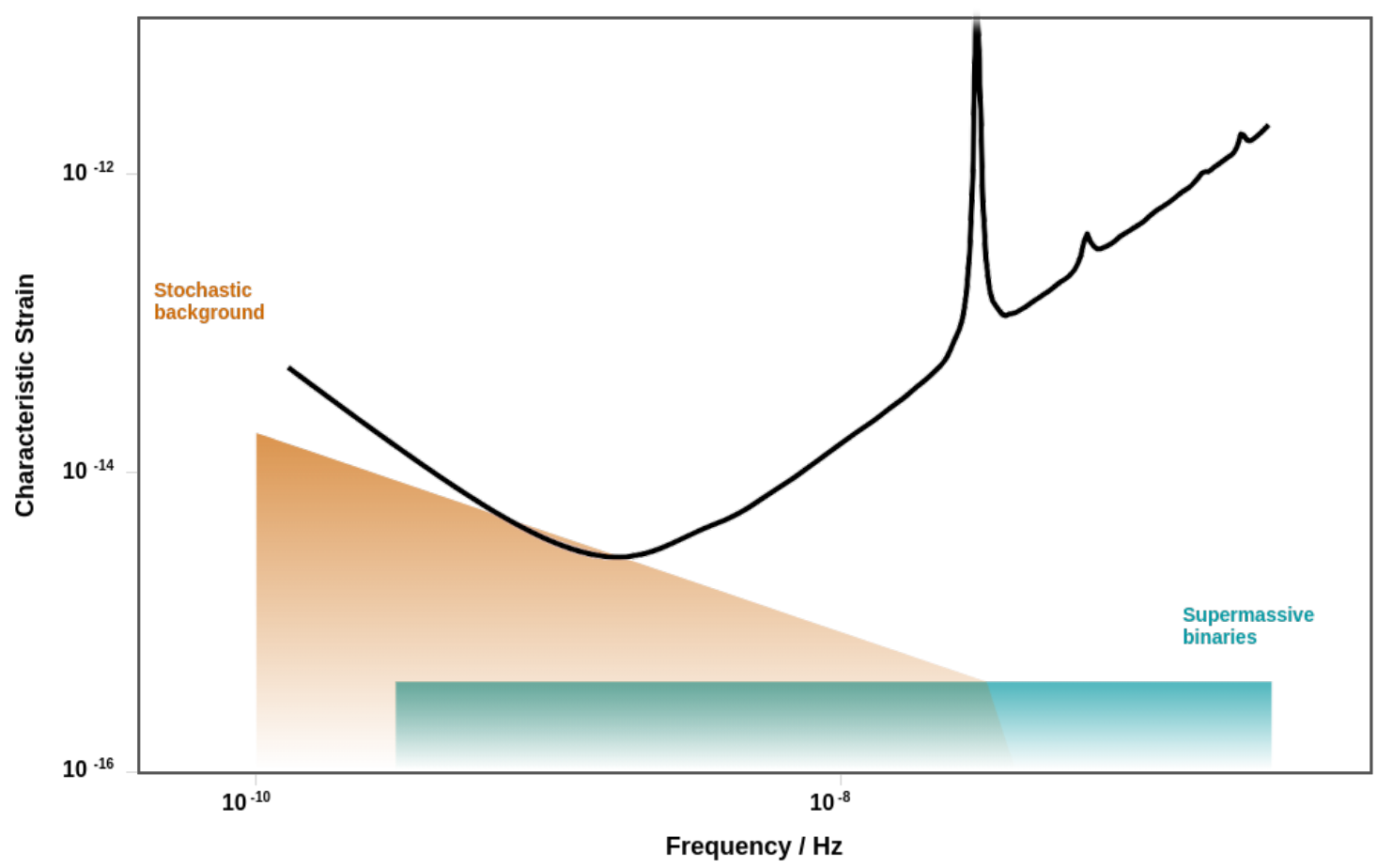

Figure 2.9: Simulated noise curve based on the 12.5 year dataset including the white noise parameters. The shaded indicate the characteristic strain amplitude of the stochastic background and individual SMBHB sources. The noise curve was generated by Jeff Hazboun using Hazboun et al. (2019) and this was adapted from gwplotter.com developed by Moore et al. (2015). 
depends on the amplitude of the stochastic background, the spindown of the pulsars, and the length of the dataset. The curve is less sensitive at these low frequencies because both the linear and quadratic fitting done for period and period derivative, respectively, in the timing process described above remove GW power on timescales on the order of the length of the data set. By observing more pulsars for longer periods of time, it is possible to make the PTA more sensitive to the stochastic background and also individual GWs from SMBHBs.

Properly accounting for noise features in the data increases the sensitivity towards a passing GW. When a plane-wave GW passes through our galaxy, it alters the space-time at Earth and at the pulsar at different times. The apparent rotational frequency of each pulsar being observed from Earth has a Doppler-shift given by $f_{\text {obs }}=(1+z) f_{\text {emit }}$ as measured from the solar system barycenter, where the redshift $z$ is defined as

$$
z(t)=\frac{\hat{p}^{i} \hat{p}^{j}}{2(1+\hat{\Omega} \cdot \hat{p})}\left[h_{i j}(t)-h_{i j}\left(t-t_{l}\right)\right] .
$$

Equation 2.5 is a function of time that is dependent on $\hat{p}$ which is the vector pointing from the Earth to the pulsar, $\hat{\Omega}$ which is a vector that points along the direction of propagation of the GW signal, and $h_{i j}$ which is the amplitude of the passing GW plane wave (Burke-Spolaor et al., 2019). Equation 2.5 is derived from integrating from the pulsar to the Earth, so the quantity $h_{i j}(t)$ is the strain measured at Earth and the quantity $h_{i j}\left(t-t_{l}\right)$ is the strain measured at the pulsar. The time $t_{l}$ is defined as $t_{l}=(l / c)(1+\hat{\Omega} \cdot \hat{p})$, where $l$ is the distance from the pulsar to the 
solar system barycenter. The difference in time between the GW passing Earth and the pulsar gives rise to what are known as the "Earth" and "pulsar" terms. The Earth term is the measurement of the signal from the source to the Earth, while the pulsar term is the measured signal from the source to the pulsar to the Earth $\left(t_{l}\right)$. This forces the pulsar term to always represent a snapshot of the signal's evolution as it was in the past, because of the additional amount of time it takes for the signal from the source to reach the pulsar and then reach the Earth. If both terms can be detected, using information from both terms helps to describe the evolution of the binary, giving a more complete picture of the GW signal.

\subsection{Motivation and Challenges of Detecting Eccentric BBH Sources}

\subsubsection{In the Ground-Based Regime}

As mentioned in Section 1.1 and further in Section 1.2.2, there are two types of environments that stellar-mass BBHs can form in: isolated or dynamic. Eccentricity is one of several GW source parameters that can differentiate source populations from either environment. Several studies of LIGO-Virgo detections covering the first and second observing runs show that the GW sources found are consistent with little to no eccentricity, $e_{10 \mathrm{~Hz}}<0.1$ Romero-Shaw et al. 2019; Wu et al. 2020; O'Shea \& Kumar 2021 where $e_{10 \mathrm{~Hz}}$ represents the measured eccentricity at a reference GW frequency of $10 \mathrm{~Hz}$. However, measurements of the eccentricity of GW190521 and GW190620A strongly suggest it contains $e_{10 \mathrm{~Hz}}>0.1$ (Gayathri et al., 2020; Romero-

Shaw et al., 2020, 2021), providing a strong argument for the inclusion of eccentricity 
in current low-latency searches.

As mentioned in Section 2.1.1.1, matched-filtering is one of the main methods utilized on ground-based GW data and is a highly-sensitive search method. It is expected that a large fraction of the eccentric source population will enter the LIGOVirgo band with $e_{10 \mathrm{~Hz}} \lesssim 0.1$. Even within this low-eccentricity population, there is a small fraction $(\sim 5 \%)$ of sources that are not detectable using quasi-circular waveform models. For sources with $e_{10 \mathrm{~Hz}}>0.1$, eccentric waveform models are needed to detect the complete population (Zevin et al., 2021). Even when eccentric sources are detectable using quasi-circular waveforms, the mismodeling can bias parameter estimation (O'Shea \& Kumar, 2021).

Currently, there are a variety of waveforms that use eccentricity in their models (Moore \& Yunes, 2019; Moore et al., 2016; Cao \& Han, 2017; Huerta et al., 2017; Tiwari et al., 2019; Tanay et al., 2016; Hinder et al., 2018; Huerta et al., 2014, 2018; Nagar et al., 2021). Of the available eccentric waveforms, only a few have been implemented into LALsuite (LIGO Scientific Collaboration, 2018) that model the inspiral phase of the signal only, lacking the merger and ringdown (e.g. Huerta et al., 2014; Moore et al., 2016; Tanay et al., 2016). Not having a complete waveform template can exclude information that is important for constraining the source parameters of BBH systems. The dearth of eccentric waveforms make it difficult to conduct template-based searches for eccentric BBHs, especially highly eccentric BBHs.

In addition to matched-filter searches, LIGO/Virgo also employs unmodeled burst search methods, like BayesWave and cWB that were discussed in Section 2.1.1.1. 
As there are a lack of reliable eccentric waveforms implemented into LALsuite, LIGO/Virgo used cWB to search for eccentric BBH in $\mathrm{O} 1$ and $\mathrm{O} 2$ data. With cWB, they recovered seven out of the ten BBH detections, which they believe to be consistent with little to no eccentricity, as in the template-based search. No new eccentric BBH candidates were identified (Abbott et al., 2019c). As previously noted, these burst searches are not as sensitive as a template-based search, but they are not constrained by the use of waveform models in their analyses; still, they are not optimal for eccentric binary detection.

\subsubsection{In the PTA Regime}

Similarly to the ground-based regime, detecting sources with eccentricity in the PTA regime will help us better understand the process of inspiraling SMBHBs. While the origin of the data, noise sources in the data, and sources of GWs are very different to that of ground-based detectors, both share the same property in that the resulting data is a time series with underlying GW signal. Thus, the burst-like appearance of an eccentric signal can still be seen in the timing residuals, particularly at higher eccentricities (Figure 2.10). However, the main difference is that the time scales are much longer since the evolution of SMBHBs is slower compared to their stellar mass counterparts. Figure 2.10 compares residuals from a circular source to that of an eccentric one. For a signal from a circular binary, the Earth and pulsar term contributions are fairly similar but in the case of the highly eccentric signal, there is a stark difference between the two terms. This is due to the pulsar term 

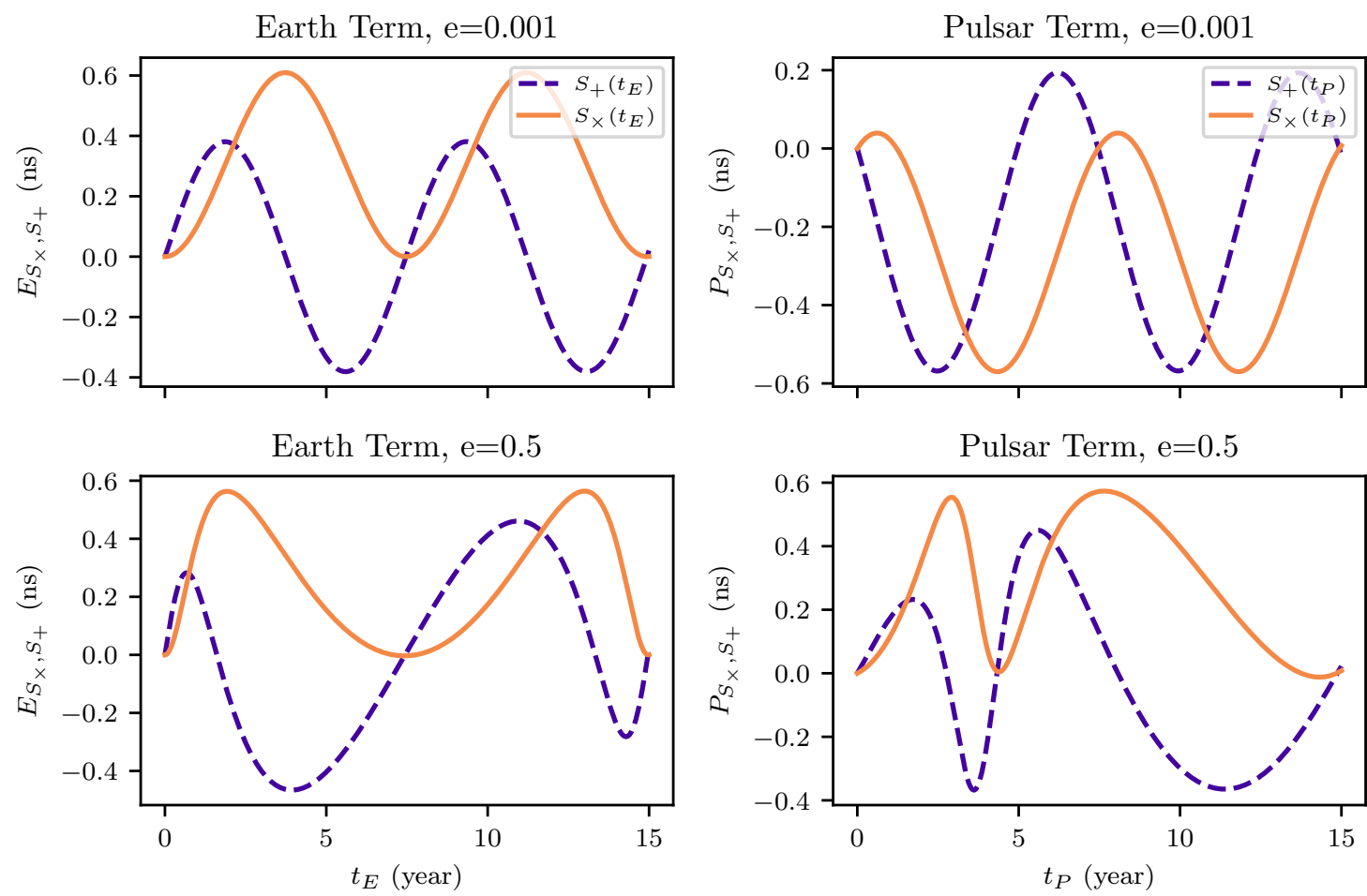

Figure 2.10: Residuals from a quasi-circular source vs an eccentric source for both polarization modes. The left column shows the difference between a quasi-circular source and eccentric source in the Earth term. The right column shows the difference between a quasi-circular source and eccentric source in the pulsar term. Plot created using code developed by Susobhanan et al. (2020). 


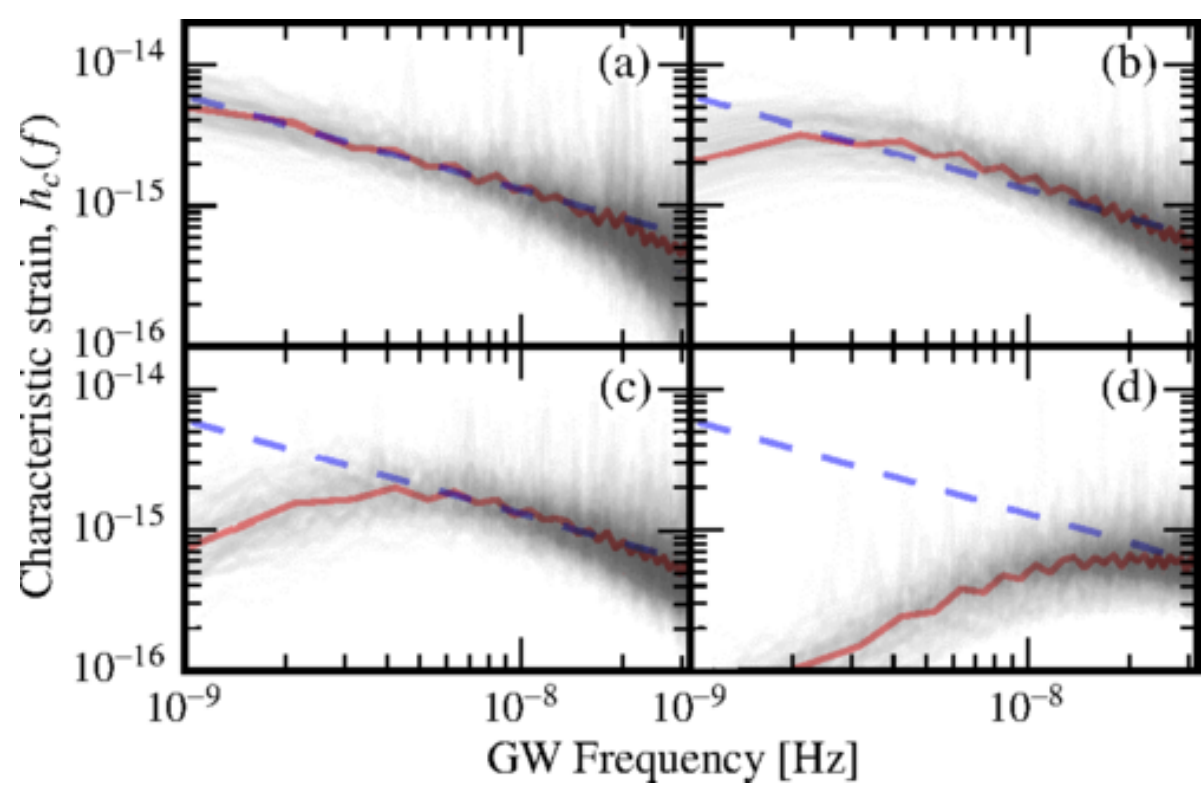

Figure 2.11: This figure shows simulated characteristic strain spectra for different stochastic background populations under different astrophysical conditions. The grey lines represent single population realizations and the red line is the mean of those realizations. The blue dashed line represents $f^{-2 / 3}$ strain spectrum as a reference. Each panel varies in initial orbital eccentricity $\left(e_{0}\right)$ and the mass density of the stars at the gravitational influence radius of the binary $(\rho)$. a) $e_{0}=0, \rho=10 M_{\odot} \mathrm{pc}^{-3}$; b) $e_{0}=0.95, \rho=10 M_{\odot} \mathrm{pc}^{-3}$; c) $\left.e_{0}=0, \rho=10^{4} M_{\odot} \mathrm{pc}^{-3} ; \mathrm{d}\right)$ $e_{0}=0.95, \rho=10^{4} M_{\odot} \mathrm{pc}^{-3}$ Figure credit: Taylor et al. (2017)

containing information on the binary much earlier in its evolution and the Earth term contains information on the current state of its evolution. This shows that the contribution from the pulsar term is especially important when it comes to studying eccentric sources.

From looking at how orbital eccentricity can affect the appearance of a signal from a single SMBHB, it can be inferred that eccentricity can also have an effect on the stochastic background. As we know, eccentricity mostly impacts the inspiral phase of a binary and the distribution of GW power being radiated from a binary (See Section 1.2.1). Since PTAs will mostly be sensitive to the inspiral phase of SMBHBs, these effects are important to note. Figure 2.11 shows the effect of the 
initial orbital eccentricity $\left(e_{0}\right)$ and the mass density of the stars gravitationally influenced by a SMBHB $(\rho)$ for a population of signals generated by inspiraling SMBHBs. It's clear from looking at panel d from Figure 2.11 that high $e_{0}$ and $\rho$ combined can have a significant effect on the appearance of the stochastic background. This is due to highly eccentric systems evolving much quicker than quasi-circular systems. This faster evolution results in more binaries emitting at higher GW frequencies than at lower frequencies.

So far, there has been more effort placed in searching for circular GW sources (Aggarwal et al., 2019; Arzoumanian et al., 2020a). Signals from circularized SMBHBs are easier to model and search for in PTA data compared to eccentric systems which are difficult to simulate since they're computationally expensive and take a long time to run (Vogelsberger et al., 2014; Fitts et al., 2017). Although eccentric systems are inherently more difficult to model, efforts have been made to make codes that can compute residuals from eccentric sources (Taylor et al., 2016; Susobhanan et al., 2020) which can be used to develop a search pipeline for eccentric GWs. 


\section{Chapter 3}

\section{Method for Detecting Highly-Eccentric Binaries with a}

\section{Gravitational-Wave Burst Search}

As mentioned in Section 2.1.1.2, a plethora of GW transient events detected by the aLIGO and Advanced Virgo observatories have given us a wealth of understanding about compact binary dynamics, compact binary formation, and the stellar graveyard population. To date, all of the GW events published in the GW transient catalogs have come from quasi-circular, compact binary sources (Abbott et al., 2021a). Meaning that these binaries entered the aLIGO/Virgo band ( $\gtrsim 10 \mathrm{~Hz}$ ) with little to no eccentricity.

From Section 1.1 we know that when it comes to stellar mass BBH systems their various formation pathways can stem from two types of environments: isolated and dynamic. We also know that eccentricity could be used as the discerning factor to determine the formation pathways used by a BBH system. Section 2.2.1 discussed that while matched-filtering is highly sensitive, it needs fairly accurate waveform models to properly explore the parameter space. With the lack of reliable, publicly available eccentric waveform models, it makes it quite difficult to formulate a search using matched-filtering.

In Section 2.2.1 we noted other search methods known as burst search methods like BayesWave and CWB that are also utilized by the LIGO-Virgo-Kagra Scientific 
Collaboration (LVK). These methods are designed to search for excess power in compact regions in time and frequency space. With this in mind, we decided to test how well a burst search method can locate bursts associated with an eccentric GW. For these tests we utilize BayesWave.

\subsection{Initial Testing with BayesWave}

As discussed in Section 2.1.1.1, BayesWave is one of the burst search methods implemented by the LVK to search for signals in GW data. It utilizes wavelets to locate burst-like features in GW data and it is this aspect of BayesWave that we wanted to utilize in locating bursts associated with an eccentric GW signal.

To construct our dataset for the injection analysis, we used EccentricTD (Tanay et al., 2016) to construct waveforms that would be injected into white gaussian noise. EccentricTD is an inspiral-only, time-domain waveform that produces signals for an eccentric, non-spinning BBH system. Since EccentrictD could only accurately produce waveforms up to $e \leq 0.4$, we could only test for lower eccentricities. An example of how well BayesWave recovered these signals can be seen in Figure 3.1. This Figure shows the Gaussian noise in light grey, the original signal in green, and the reconstructed signal in purple.

From these tests, it was clear that BayesWave found the "louder" parts of the signal as it was designed to do, but missed the "quieter" parts of the signal (Cheeseboro et al., 2017). This is because BayesWave, like other burst search methods, was designed to look for excess power in compact regions of time and frequency 

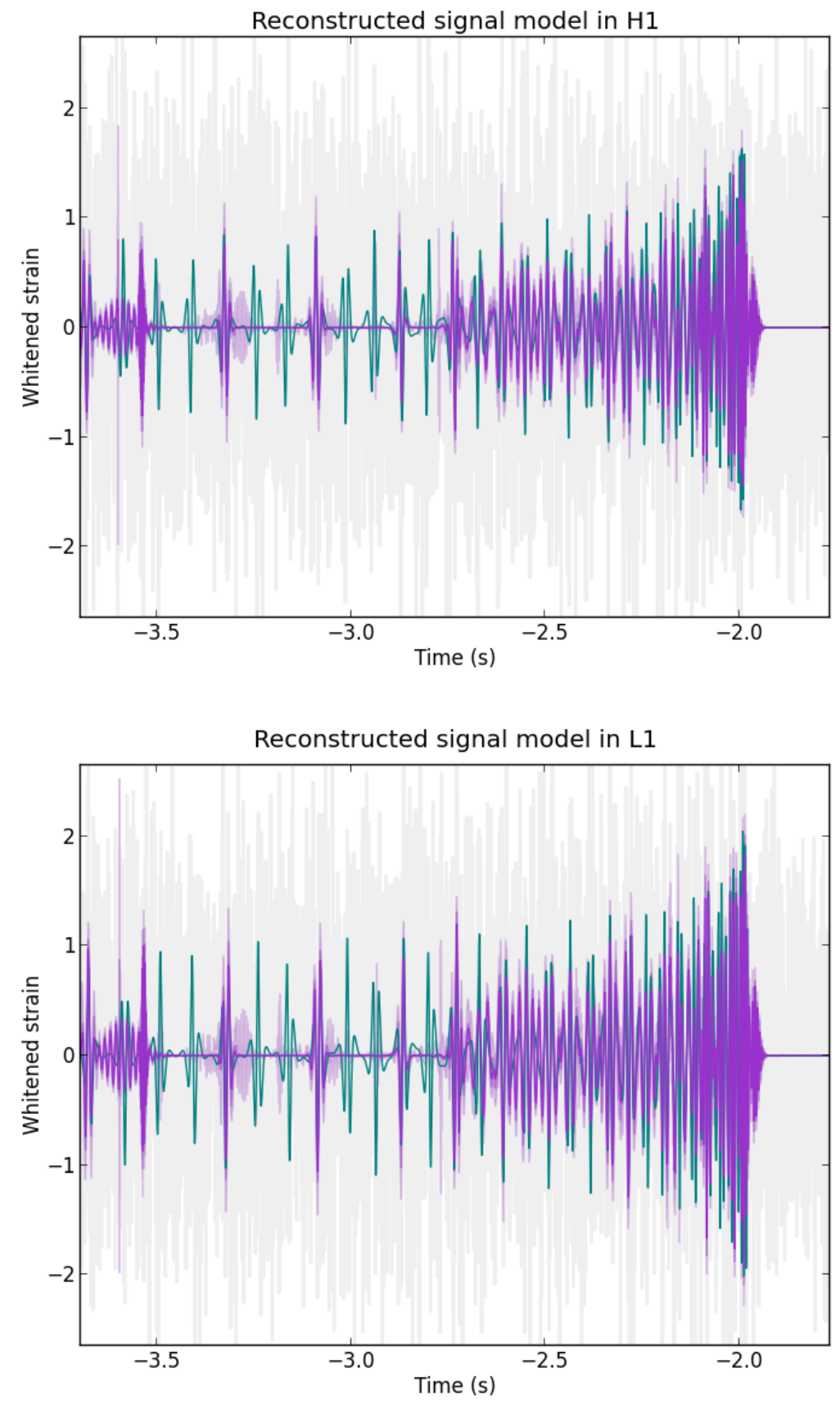

Figure 3.1: This plot shows the recovered signal from a simulated eccentric GW with $e_{0}=0.4$ that was injected into both detectors marked as $\mathrm{H} 1$ for Hanford (top) and L1 for Livingston (bottom). The light grey represents the gaussian noise, the green represents the injected signal, and purple represents the reconstructed signal recovered by BayesWave. This plot was generated using BayesWave. 
space. This makes it a bad fit for detecting the temporally disconnected bursts from highly eccentric BBH. If it is possible to "tune" BayesWave to treat these temporally disconnected bursts as being from a common source, then it will make BayesWave and other burst search methods more effective at detecting GW signals from highly eccentric BBHs.

Therefore, we developed an eccentric burst prior that could be implemented in these burst search methods to search for and connect temporally disconnected bursts from highly eccentric BBH. This eccentric burst prior is largely based on the work done by (Loutrel \& Yunes, 2017) for their Newtonian Burst Model. In Section 3.2 we describe the process of redefining the forward moving equations from Loutrel \& Yunes (2017) to create backward moving equations. We also define our prior model and describe the signal model that we used for testing our prior. Next, in Section 3.3 we discuss the simulated data and methods used for testing our prior along with the results of these tests. Finally, we discuss the results of our tests with the eccentric burst prior in Section 3.4

\subsection{Modeling Bursts from Highly Eccentric Sources}

Our method is based on the model described by L\&Y 2017. They model the GWs emitted by highly eccentric binaries as a series of discrete GW bursts. The bursts are assumed to be emitted instantaneously at each pericenter crossing of the system with negligible emission occurring during the rest of the orbit. In the Newtonian burst model the system follows a series of Keplerian orbits. The orbital 
parameters are constant throughout each orbit, updating after the burst of GWs is emitted. The instantaneous eccentricity refers to the eccentricity of the current orbit used to determine the GW emission at a particular pericenter crossing.

Each GW burst is assumed to be compact in time and frequency. L\&Y 2017 represent each burst as a rectangular tile in time-frequency space, characterized by its centroid, $t, f$, and two widths, $\sigma_{t}, \sigma_{f}$. They evolve the binary system forward in time using a slow speed post-Newtonian expansion. Given the time-frequency location of an initial burst of GWs, their model uses the total mass of the binary, $M$, pericenter separation, $r$, and instantaneous eccentricity, $e$, to determine the time until the next burst, $\Delta t$, and update the orbital parameters $r$ and $e$ for the next orbit. These orbital parameters also determine the GW frequency of each burst.

As a proof of concept, our method is based on the lowest order evolution presented by L\&Y 2017: Keplerian orbits perturbed by the instantaneous emission of quadrupolar gravitational radiation at pericenter. Instead of using rectangular timefrequency tiles, we represent each burst as a single Morlet-Gabor wavelet, imitating BayesWave (Cornish \& Littenberg, 2015). We use the evolution model of L\&Y 2017 to place a joint prior on the centroids of all wavelets. Model wavelets that are located in time-frequency space according to the L\&Y 2017 evolution are assigned high prior probability, while low prior probability is assigned to wavelets elsewhere (e.g. between two pericenter crossings).

This results in a signal description that is not a fully phase-connected waveform model. The model we present has more freedom to overcome systematic waveform uncertainties at the expense of some lost signal power. 


\subsubsection{Centroid Mapping Equations}

L\&Y 2017 defines the orbital parameters of one burst in terms of the burst that directly precedes it. Their expansion relies on two small parameters associated with pericenter separation $\frac{M}{r} \ll 1$ and eccentricity $\delta e=1-e \ll 1$. For the remainder of this section we will work in geometrized total mass units, where $G=c=1$ and the binary total mass $M=1$. Chirp mass $\mathcal{M}$, distance $r$, and time $t$ are measured in units of $M$ and frequency in units of $M^{-1}$.

The forward orbital evolution of a highly eccentric binary system is presented in section 2.1 of L\&Y 2017, to leading order. This gives the orbital parameters associated with the next $(i+1)$ GW burst in terms of the previous $(i)$. In addition to the forward evolution, we want to know the backwards evolution of the system. If we detect one burst from a highly eccentric binary, we want to search for other GW bursts produced by the same system. To obtain the backwards evolution we can invert their solution, solving for the previous $(i-1)$ burst in terms of the next (i). Working to first order

$$
\begin{aligned}
\delta e_{i \pm 1} & \approx \delta e_{i} \pm \frac{85 \pi \sqrt{2}}{12} \mathcal{M}^{5 / 3} r_{i}^{-5 / 2}\left(1-\frac{1718}{1800} \delta e_{i}\right) \\
r_{i \pm 1} & \approx r_{i}\left[1 \mp \frac{59 \pi \sqrt{2}}{24} \mathcal{M}^{5 / 3} r_{i}^{-5 / 2}\left(1+\frac{121}{236} \delta e_{i}\right)\right] .
\end{aligned}
$$

The orbital evolution of the binary can be mapped to the GW signal evolution in time-frequency space. In the Newtonian burst model the time between bursts is 
the time between pericenter crossings, i.e. the orbital period. The burst frequency is set by the instantaneous orbital frequency defined by the pericenter distance and orbital speed $2 \pi f_{G W} \sim \frac{v}{r}$. The period and pericenter speed are given by Kepler's laws to leading order, so

$$
\begin{aligned}
t_{i} & \approx t_{i-1}+2 \pi \sqrt{\frac{r_{i}^{3}}{\delta e_{i}^{3}}} \\
2 \pi f_{i} & \approx \sqrt{\frac{\left(2-\delta e_{i}\right)}{r_{i}^{3}}}
\end{aligned}
$$

Recall that $M=1$ in our total mass units. In both relations of Equation 3.2 the orbital parameters of the $i$ th burst on the right-hand-side are determined from the previous burst using Equation 3.1.

Given the GW parameters of a burst, we first determine the orbital parameters. We then evolve the orbital parameters to the next (or previous burst) and finally determine the GW parameters of that burst.

For our analysis, any burst can be fully characterized by three parameters: its central time, central frequency, and instantaneous eccentricity. (The pericenter distance makes a convenient computational midpoint, but we prefer to work directly with GW frequency in the model.) If these three parameters are known for any one burst, the binary's chirp mass will uniquely determine all other bursts where our leading order approximations are valid. This allows us to directly write the leading order evolution in terms of only $t, f$, and $\delta e$. Given the $i$ th burst the next burst in 
the sequence is

$$
\begin{aligned}
t_{i+1} & =t_{i}+\frac{1}{f_{i}} \sqrt{\frac{2-\delta e_{i}}{\delta e_{i}^{3}}} \\
f_{i+1} & =f_{i}\left[1+\frac{23}{2}\left(\frac{\pi}{2}\right)^{2 / 3}\left(\pi f_{i} \mathcal{M}\right)^{5 / 3}\left(1+\frac{7547}{4140} \delta e_{i}+\frac{3725}{552}\left(\frac{\pi}{2}\right)^{2 / 3}\left(\pi f_{i} \mathcal{M}\right)^{5 / 3}\right)\right] \\
\delta e_{i+1} & =\delta e_{i}+\frac{85}{3}\left(\frac{\pi}{2}\right)^{2 / 3}\left(\pi f_{i} \mathcal{M}\right)^{5 / 3}\left(1-\frac{121}{225} \delta e_{i}\right)
\end{aligned}
$$

and the previous burst in the sequence is

$$
\begin{aligned}
t_{i-1} & =t_{i}-\frac{1}{f_{i}} \sqrt{\frac{2-\delta e_{i}}{\delta e_{i}^{3}}} \\
f_{i-1} & =f_{i}\left[1-\frac{23}{2}\left(\frac{\pi}{2}\right)^{2 / 3}\left(\pi f_{i} \mathcal{M}\right)^{5 / 3}\left(1+\frac{7547}{4140} \delta e_{i}+\frac{5875}{368}\left(\frac{\pi}{2}\right)^{2 / 3}\left(\pi f_{i} \mathcal{M}\right)^{5 / 3}\right)\right] \\
\delta e_{i-1} & =\delta e_{i}-\frac{85}{3}\left(\frac{\pi}{2}\right)^{2 / 3}\left(\pi f_{i} \mathcal{M}\right)^{5 / 3}\left(1-\frac{121}{225} \delta e_{i}-\frac{295}{12}\left(\frac{\pi}{2}\right)^{2 / 3}\left(\pi f_{i} \mathcal{M}\right)^{5 / 3}\right) .
\end{aligned}
$$

Ideally, the forward and backward evolution would be perfect inverses of each other. However, because we truncate the evolution to low order, this is not the case. Owing to the low order approximation, we find the error in the time-frequency evolution to be less than $10 \%$ for $e \gtrsim 0.7$ and $r \gtrsim 15 M$. 


\subsubsection{Eccentric Burst Prior}

The centroid mapping procedure tells us where bursts are expected to occur. We wish to construct a prior for the observed burst centroids given their expected locations. The eccentric burst prior consists of a sum of bivariate normal distrubutions, each centered on an expected burst centroid. The covariance of each peak, $\Sigma_{i}$, represents the uncertainty in the wavelet being centered at that location.

The prior is defined by a set of five meta-parameters: the binary total mass, $M$, and chirp mass, $\mathcal{M}$, the GW signal parameters of one burst, $\left(t_{\star}, f_{\star}\right)$, referred to as the "anchor burst", and the instantaneous eccentricity of the system for the orbit that produced the anchor burst $\delta e_{\star}$.

$$
\begin{aligned}
p\left(t, f ; M, \mathcal{M}, t_{\star}, f_{\star}, \delta e_{\star}\right) & =\frac{1}{2 \pi N} \sum_{i=0}^{N} \frac{1}{\operatorname{det} \Sigma_{i}} e^{-\mathbf{d}_{i} \Sigma_{i}^{-1} \mathbf{d}_{i}^{T}} \\
\mathbf{d}_{i} & =[t, f]-\left[t_{i}, f_{i}\right],
\end{aligned}
$$

where $N$ is the number of bursts expected in the observation time to be analysed. The total mass does not appear in the centroid mapping Equation 3.1. It is effectively a unit conversion factor, converting the burst $t, f$ from the S.I. units of our analysis to the total mass units of the centroid mapping equations.

Starting from the covariance of the anchor burst peak, $\Sigma_{\star}$, the uncertainty can be propagated through the centroid mapping equations using a Jacobian. This allows us to determine the covariance of all other peaks in the prior distribution. To account for the dependence of $t$ and $f$ on $\delta e$, we compute the covariance evolution 
in three dimensions, but only use the appropriate 2D block when constructing the prior.

$$
\begin{array}{r}
\mathbf{x}=(t, f, \delta e) \\
J_{ \pm, m n}=\frac{\partial x_{m, i}\left(\mathbf{x}_{i \pm 1}\right)}{\partial x_{n}} \\
\Sigma_{i}=J_{ \pm} \Sigma_{i \pm 1} J_{ \pm}^{T},
\end{array}
$$

where $i$ is the index of the peak (i.e., which burst) and the $m, n$ indices of the Jacobian matrix run through the three burst parameters. $x_{m, i}$ are the parameter evolution equations, Equation 3.3 and Equation 3.4, which are functions of the $(i \pm 1)$ th parameters. The full functional forms of $J_{ \pm}$are presented in Section 3.5.

A 2D time-frequency map of the prior is shown in Figure 3.2 for a choice of meta-parameters. For these parameters, three bursts are expected to occur during the $3 \mathrm{~s}$ analysis window. More bursts should occur both before and after these three, but they do not contribute to the prior. The central peak is the location of the anchor burst, and is vertically aligned. The Jabobian evolution causes the $t, f$, and $\delta e$ uncertainties to mix introducing $t$ - $f$ covariance in the other peaks. Intuitively, the tilting of the outer peaks toward the center is primarily driven by the uncertainty in $f$. High frequency sources will have less time between bursts and low frequency sources will have more time. The time between the high frequency edges of the peaks is reduced, relative to the centroid and the time between the low frequency edges is increased.

In practice $\left(t_{\star}, f_{\star}\right)$ should closely follow the $(t, f)$ of one detected burst. There 


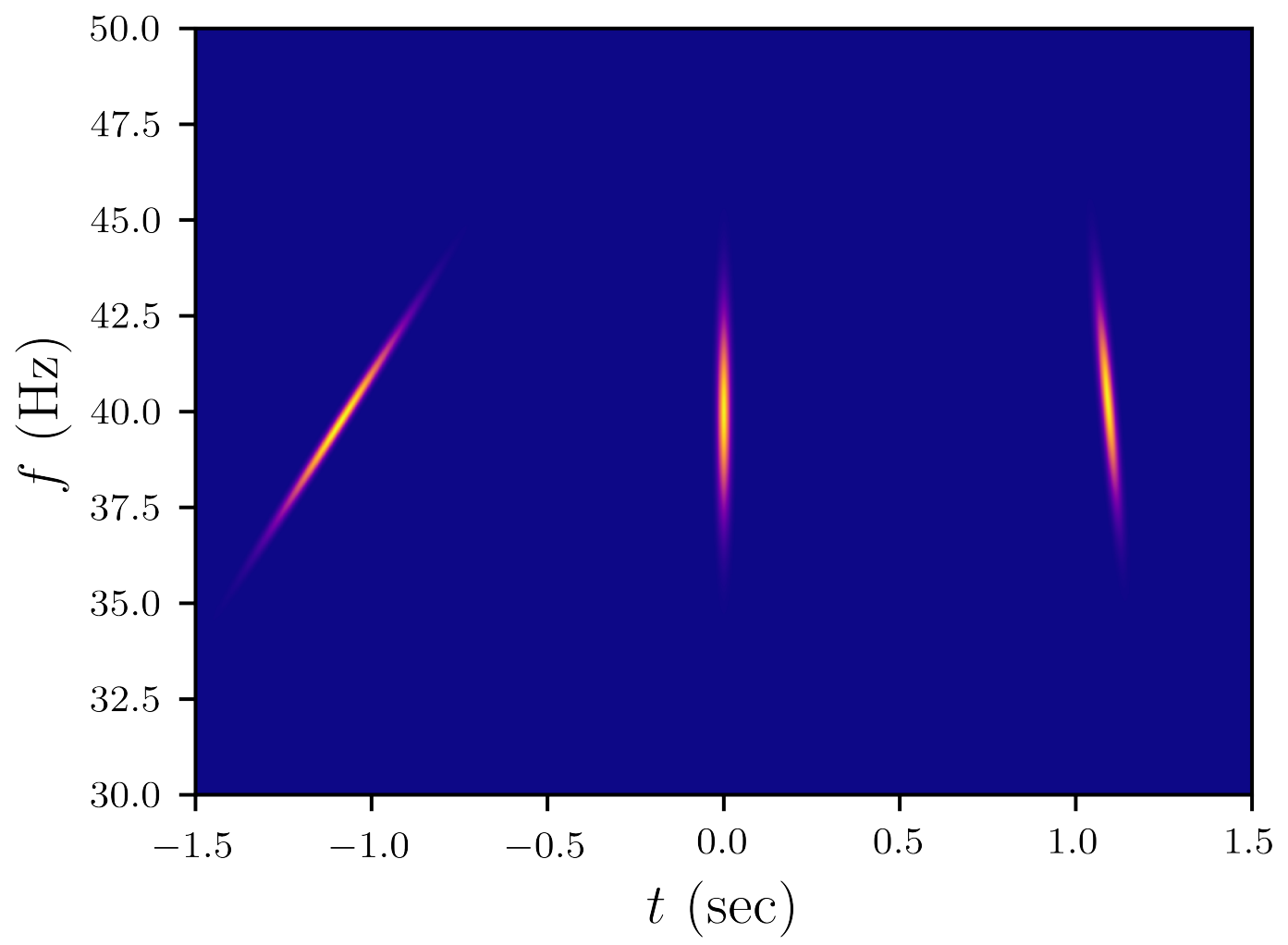

Figure 3.2: Prior probability for the time-frequency location $(t, f)$ of the centroid of a GW burst for the meta-parameters $M=50 M_{\odot}, \mathcal{M}=15 M_{\odot}, t_{\star}=0, f_{\star}=40 \mathrm{~Hz}$. $\delta e_{\star}=0.1$. In this case the prior preference is to have three bursts in the analyzed time window. The full prior is the sum of three bivariate normal peaks, one for each expected burst (Cheeseboro \& Baker, 2021).

is some ambiguity in which is the anchor burst. Assuming the centroid mapping is approximately symmetric, any burst in the series could act as the anchor burst with relatively even probability. This leads to the meta-parameter distributions being multimodal and presents a practical challenge when sampling the space.

\subsubsection{Signal Model}

To accompany the eccentric burst prior, we require a GW burst signal model. We implement a simplified version of the BayesWave model. BayesWave models 
excess detector power using Morlet-Gabor wavelets (Cornish \& Littenberg, 2015). Our signal model assumes the GWs emitted at each pericenter crossing can be fit with a single wavelet. Each wavelet is defined by five parameters,

$$
\begin{aligned}
\Psi_{i}\left(t ; t_{i}, f_{i}, A_{i}, Q_{i}, \phi_{i}\right)=A_{i} \exp \left[-\left(\frac{2 \pi f_{i}}{Q_{i}}\right)^{2}\left(t-t_{i}\right)^{2}\right] & \\
& \times \cos \left[2 \pi\left(t-t_{i}\right)+\phi_{i}\right]
\end{aligned}
$$

where $A_{i}$ is its amplitude, $Q_{i}$ its quality factor, $\phi_{i}$ its reference phase, and $t_{i}$ and $f_{i}$ are the central time and frequency of the wavelet. The subscript $i$ refers to the individual wavelets in the model, where the full signal model is the sum of all wavelets.

The eccentric burst prior is a prior on the wavelets' time-frequency centroids, $t_{i}$ and $f_{i}$. A given signal model will have a number of $(t, f)$ pairs equal to the number of wavelets in the model. The expectation is that each high probability peak in the eccentric burst prior should have one wavelet nearby. The eccentric burst prior associates multiple temporally disconnected bursts into a single GW signal with one wavelet per burst. This goes against the usual GW burst search techniques which focus on reconstructing a single GW burst with multiple wavelets that are nearby in time-frequency space.

The GW bursts emitted during pericenter crossings of a highly eccentric orbit contain only one GW cycle. We expect the recovered wavelets to have low quality factors $Q \sim 2$, although we do not formally enforce this expectation with a prior on 
Q. BayesWave uses a Reversible Jump Markov Chain Monte Carlo (RJMCMC) to sample not only the individual wavelet parameters but also the number of wavelets in the model. Implementing a comparible RJMCMC scheme to BayesWave is beyond the scope of this proof of concept study. For simplicity, we opt to fix the number of wavelets in our signal model.

\subsection{Analysis of Simulated Data}

To test our eccentric burst prior method, we simulate single detector LIGO/Virgolike data. This simulated data does not incorporate the response of any particular detector, assuming an optimally oriented source and a white noise spectrum across the whole range of frequencies simulated.

The injected GW signals cover a narrow frequency band, so the lack of a realistic noise spectrum is not a major issue. We use a Gaussian, white noise likelihood. While the eccentric burst prior is not as prescriptive as a phase-connected waveform, it still works best when the small parameter conditions of the centroid mapping series are met. These conditions are satisfied for GW bursts emitted at earlier times of the inspiral. The simulated signals are truncated to contain three GW bursts emitted well enough before merger that these conditions are met. In its current form our model would have trouble with bursts emitted closer to merger. The approximations used to determine Equation 3.3 and Equation 3.4 would not be valid. Additionally, the wavelets would preferentially fit the higher amplitude merger, missing the lower amplitude early inspiral. 
Since each simulation contains three bursts, we fix the number of wavelets in the BayesWave-like signal model to three.

We perform two sets of simulations using both a uniform prior and the eccentric burst prior. The first uses simple waveforms constructed from three wavelets spaced according to the centroid mapping equations of Section 3.2.1. These simple waveforms contain bursts that are roughly 20 orbits before merger. The second uses a more realistic waveform model for highly eccentric BBHs (East et al., 2013). The bursts in this set are roughly 80 orbits before merger. In each case we use sources with an initial eccentricity of $e=0.9, \delta e=0.1$ and cover $\mathrm{GW}$ frequencies of $\sim 30-65 \mathrm{~Hz}$

For both the eccentric burst prior and uniform prior cases, we place uniform priors on the wavelets' quality factors $(1.5 \leq Q \leq 15)$, phases $(-\pi \leq \phi \leq \pi)$, and amplitudes $(A>0)$. The uniform priors for the wavelets' central time and frequency cover the entire time-frequency data volume, as set by the time span and sampling rate of the data. Each simulation contains $1.5 \lesssim T \lesssim 3$ s of data. The corresponding frequency prior is uniform on $1 / T \leq f \leq f_{\text {Nyquist }}$. The eccentric burst prior is set to zero outside the same time-frequency volume as the uniform prior.

We place uniform priors on the meta-parameters of the eccentric burst prior. Like the wavelet time and frequency parameters, the priors for anchor burst time and frequency are uniform on the whole data volume. The anchor burst eccentricity is uniform on $10^{-3} \leq \delta e \leq 0.9$. The prior for total mass is uniform on $0 \leq M \leq$ $200 M_{\odot}$. The chirp mass is held to $\mathcal{M}>0$. Because $\mathcal{M}<M$, the prior on $M$ additionally constrains $\mathcal{M}$. 
To evaluate the efficacy of the eccentric burst prior, we use a parameter estimation figure of merit (Figure 3.3). To better compare between individual simulations, we use the fractional uncertainty in recovered time $\Delta t \times f_{\text {inj }}$, where $\Delta t$ is the standard deviation of the marginal posterior distribution for the wavelet central time and $f_{\text {inj }}$ is the injected GW frequency.

In the low signal-to-noise ratio (SNR) limit any GW signal should be undetectable, regardless of whether the eccentric burst prior or a uniform prior is used. In these cases the recovered $t$ should be unconstrained with its posterior filling the entirety of the prior range. The time uncertainty $\Delta t$ should be of the order of the time span of the data being analyzed, which defines the full prior range.

In the high SNR limit, a GW signal should be easily detectable, and the posterior probability distribution will be dominated by the likelihood (assuming the prior includes the maximum-likelihood probability region of the parameter space). In these cases regardless of whether one uses the eccentric burst prior or a uniform prior, the parameter estimation uncertainty should be the same, as it just the spread of the marginal posterior distribution. Between these two limits lies a transition region, where the choice of prior affects the parameter estimation uncertainty.

We choose to use the parameter estimation uncertainty as a proxy for a true detection statistic. Since the two priors, eccentric burst and uniform, have the same limiting behavior, we can examine at what SNR each prior converges to these limits. We opt for this simplified detection proxy because common information criterion statistics are unsuitable for our problem. For example the Akaike information criterion (AIC) uses the maximum likelihood and does not take into account priors. 
The deviance information criterion (DIC) is constructed from the variance of the posterior samples and is ill-defined for cases with mode switching (Gelman et al., 2014). We observe mode switching in the intermediate SNR regime of interest, for example where the sampler switches back and forth from detecting two of the injected bursts to detecting all three.

The other model parameters are not as well suited to the task of tracking detection. This is because of covariances between the parameters. In addition to time uncertainty, we present the fractional uncertainty in recovered GW frequency $\Delta f / f_{\text {inj }}$. This figure of merit has the same high-SNR limiting behaviour as $\Delta t \times f_{\text {inj }}$, where the choice of prior does not affect the uncertainty. At low SNR $f$ is not fully unconstrained by the eccentric burst prior because of the covariance between $f$ and $M$. The binary total mass effectively sets the merger frequency, so $f$ is held to lower values for high mass systems. (This effect can be seen in Figure 3.4 in the 2D marginal posterior for anchor burst frequency, $f_{\star}$, and total mass, $M$.)

\subsubsection{Simple Wavelet Injections}

The first set of simulations use injected waveforms based on the centroid mapping equations found in Section 3.2.1. Starting from a randomly selected total mass in the range $M=30-70 M_{\odot}$ and fixed mass ratio $q=0.3$ (which in turn determine the chirp mass), we use Equation 3.3 to determine the time-frequency locations of three GW bursts. We inject a $Q=2$ wavelet at each of these locations. We simulate 13 sources covering a frequency range of $f=30-60 \mathrm{~Hz}$. For each source we scale 
the amplitude to achieve a target SNR, allowing us to search for the same source at a range of SNRs from 2 to 7.

In order to keep the expected number of bursts in our analysis window at exactly three, we truncate the data before the next burst was expected. This results in unequal observation times ranging from about $2-3 \mathrm{~s}$.

We analyze each simulation twice, first with the eccentric burst prior and again using a uniform prior on the individual wavelets' time-frequency locations. We use PTMCMCSamper to draw posterior samples (Ellis \& van Haasteren, 2017). From these we determine our two parameter estimation figures of merit for each simulation. These figures of merit are summarized in the left panels of Figure 3.3, combining all trials.

As we expect, $\Delta t \times f_{\text {inj }}$ converges to the same low and high SNR limits regardless of which prior is used. For $\mathrm{SNR} \leq 3$, the injected signals are not detected. The posterior distribution for each wavelet's central time is approximately uniform across the entire observation, leading to large uncertainties in all trials. Using the eccentric burst prior, the injected signals are reliably detected at $\mathrm{SNR} \approx 5$, a lower amplitude than with the uniform prior. At the highest SNRs the posterior is dominated by the likelihood, so all bursts are detected with effectively the same fractional uncertainty.

The frequency uncertainty, seen in the lower left panel of Figure 3.3, is less clean. At higher SNRs the eccentric burst prior is more constraining than the uniform prior, meaning the posterior is not fully likelihood dominated.

Using the eccentric burst prior results in improved parameter estimation pre- 

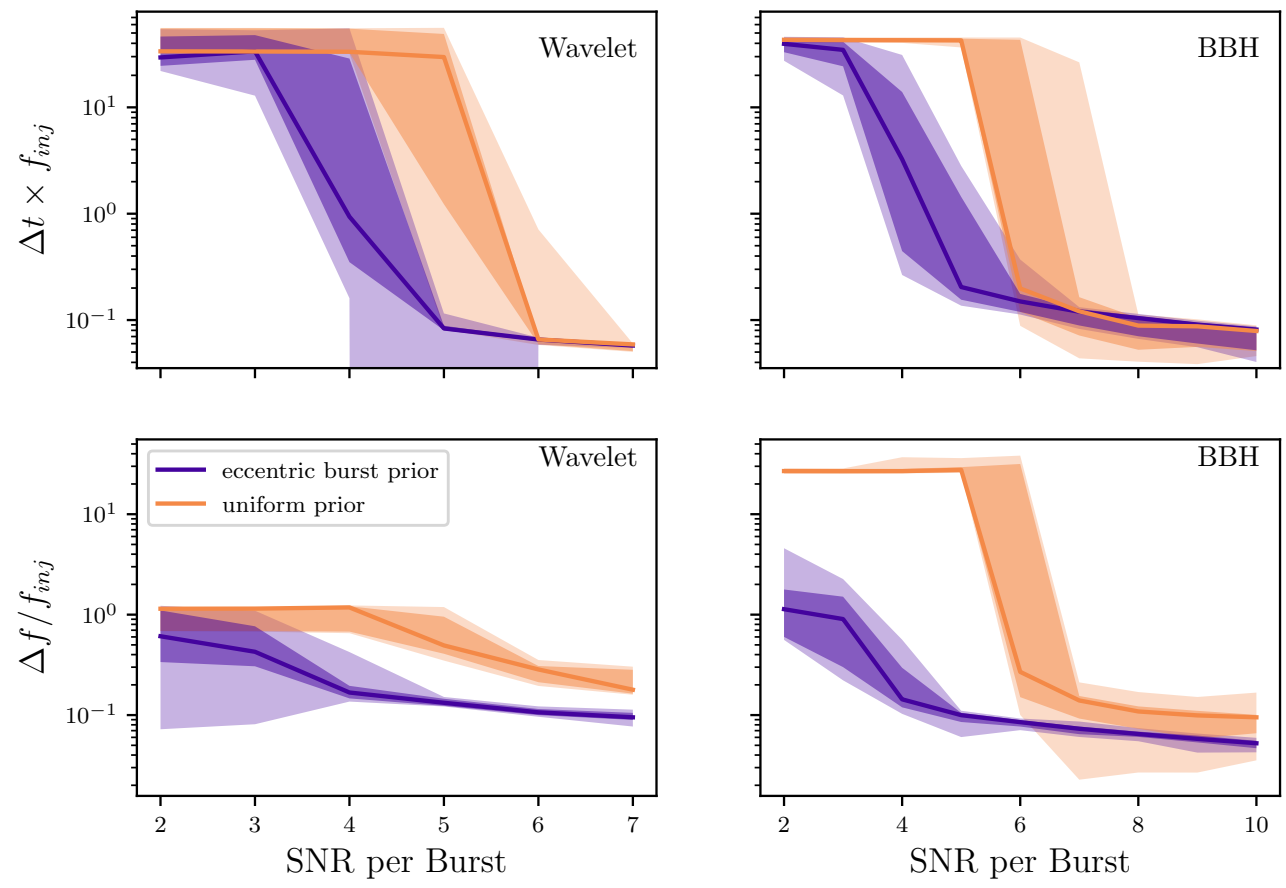

Figure 3.3: Parameter estimation uncertainty for the simple wavelet (left) and eccentric BBH waveform (right) injections as described in Section 3.3.1 and Section 3.3.2. The solid lines show the median uncertainty of all simulations, and the dark and light shaded regions represent the $68 \%$ and $95 \%$ credible intervals on the uncertainty, respectively. At low SNR the posterior distributions should fill the prior range, leading to large uncertainty. At high SNR the posterior distributions are localized leading to small uncertainty. Using the eccentric burst prior leads to better signal recovery in the intermediate SNR regime $\sim 3-6$ (Cheeseboro \& Baker, 2021)

cision in the intermediate $\mathrm{SNR}$ regime (SNR $\sim 3-6$ ), as can be seen in the upper left panel of Figure 3.3. This improved detection confidence is especially important when trying to detect quiet bursts from highly eccentric BBHs during the early inspiral phase. These initial results set a benchmark for further tests using a more realistic GW waveform. 


\subsubsection{Eccentric BBH Waveform Injections}

We follow the same basic framework as Section 3.3.1 to work with more realistic eccentric BBH waveforms from (East et al., 2013). This model maps the binary dynamics to an effective single body following a Kerr geodesic. It was designed to approximate GWs from highly eccentric systems that resulted from the radiationinduced dynamical capture of initially hyperbolic orbits (East et al., 2013). As in the simple wavelet model, we simulate multiple waveforms with initial eccentricity of $e=0.9, \delta e=0.1$. We choose an initial pericenter separation of $r=15 M$ to stay within the range of validity of the low order centroid mapping equations used in the eccentric burst prior. Unlike in the wavelet injections, we select 16 total masses in the range $M=15-30 M_{\odot}$, resulting in waveforms with $\mathrm{GW}$ frequencies of $f=30-65 \mathrm{~Hz}$. Again we truncate the waveforms to contain exactly three bursts from the early inspiral, giving observation times of $1.5-3 \mathrm{~s}$. These waveforms use a larger sampling rate by a factor of $\sim 10$ resulting in a significantly wider analysis bandwidth compared to the previous simulations.

We assume a detector orientation aligned with the plus polarization of the GWs. The waveform amplitudes were directly scaled to set the injected SNR. The waveform amplitudes were scaled to control the injected SNR by first calculating the baseline SNR of the three bursts within our time window and then taking the fraction of the desired injected SNR with the baseline SNR to create a factor to appropriately scale the signal. This is effectively equivalent to changing the distance to the source but does not account for cosmological redshift of the system mass. 
These more realistic waveforms have more structure than the wavelets used in the previous simulations. Fitting each burst with a single wavelet results in an imperfect recovery. This leads to the recovered SNR being less than the injected, so we use an extended range of SNRs compared to the simple wavelet injections, going up to 10 .

As before we analyze each simulation twice, collecting posterior samples first with the eccentric burst prior and again without. The same parameter estimation figures of merit are plotted as a function of SNR in Figure 3.3. Looking at the top right panel of Figure 3.3, we observe the same mode switch behavior as in top left panel Figure 3.3 where the sampler switches from non-detection to detection in the intermediate SNR range $3-6$. From looking at this intermediate region (SNR range $3-6$ ), we see a similar improvement in the fractional uncertainty of recovered burst central times when the eccentric burst prior is used compared to the uniform prior.

The constraining effect of the $M-f$ covariance is more apparent in this case when looking at the bottom right panel of Figure 3.3. (The $M-f$ covariance can be clearly seen in the bottom-leftmost plot in Figure 3.4.) The higher Nyquist frequency of the $\mathrm{BBH}$ waveform injections inflated the recovered frequency uncertainty in the low SNR, uniform prior case relative to the wavelet injections. At low SNR the eccentric burst prior constrains the signal frequency to stay in the low $f$ part of the search parameter space. 


\subsection{Discussion and Conclusions}

In this chapter, we developed an eccentric burst prior that can be used to improve the detection prospects for highly eccentric BBH systems when used with a GW burst search. In particular, the eccentric burst prior improves performance for intermediate SNR bursts generated during the inspiral phase of the BBH evolution, which may be missed by traditional burst methods that focus on the merger. As a proof of concept, we verified this improvement using simulated LIGO/Virgo-like data. From these simulations we found that the largest improvement over a uniform prior occurred for burst SNRs in the $3-6$ range.

The eccentric burst prior is based on a model for the time-frequency location of bursts that depend on the physical parameters of the $\mathrm{BBH}$ system. The total mass $M$ and chirp mass $\mathcal{M}$ act as meta-parameters of the prior. We hope that the posteriors for these parameters would carry useful information about the GW system producing the observed GWs. Unfortunately the recovery of these parameters was very poor, as can be seen in Figure 3.4.

Since we analyzed only three bursts from the earlier part of the inspiral of each simulation, we have limited the information to work with. The GW frequency does not evolve significantly over these three bursts, meaning we could not constrain the chirp mass $\mathcal{M}$, which relates to the frequency derivative as $\mathcal{M} \sim \dot{f}^{3 / 5}$.

The total mass $M$ would be better constrained by measuring the merger frequency, which we do not have access to. In addition to the system masses the anchor burst parameters $\left(t_{\star}, f_{\star}, \delta e_{\star}\right)$ give additional degrees of freedom. This means there 
are many combinations of meta-parameters that lead to appropriately placed bursts.

If we analyze the whole waveform all the way up to the merger, we would expect more meaningful meta-parameter recovery, but that presents additional challenges. The centroid mapping equations, Equation 3.3 and Equation 3.4, were based on the lowest order orbital evolution presented in L\&Y 2017. This low order approximation breaks down well before the merger. If we try to include bursts near the merger, the prior disagrees with the reality. The peaks in the eccentric burst prior near merger are not correctly placed. As the system approaches merger, there is significant GW emission at times other than pericenter crossing, so the isolated bursts assumption breaks as well.

L\&Y 2017 did present orbital evolution up to 3rd post-Newtonian (pN) order. With this proof of concept in place we could improve the eccentric burst prior by extending it to higher $\mathrm{pN}$ order. Improving the centroid mapping equations would allow us to probe bursts closer to the merger and better constrain the astrophysically interesting meta-parameters, $M, \mathcal{M}$, and $\delta e_{\star}$.

Also, by extending to higher $\mathrm{pN}$ order the symmetry of the centriod mapping equation is improved. When truncated to low order a step forward in the evolution equations is not the perfect inverse of a step back. This is most obvious by examining Equation 3.3a and Equation 3.4a. The time step between neighboring bursts appears identical, but when moving from the $i$ to $i+1$ burst and back, the right-hand-sides are evaluated at different bursts in the orbital sequence. Going to higher order would reduce this problem and potentially lead to better physical parameter recovery.

These two improvements would not address the fact that bursts become less 

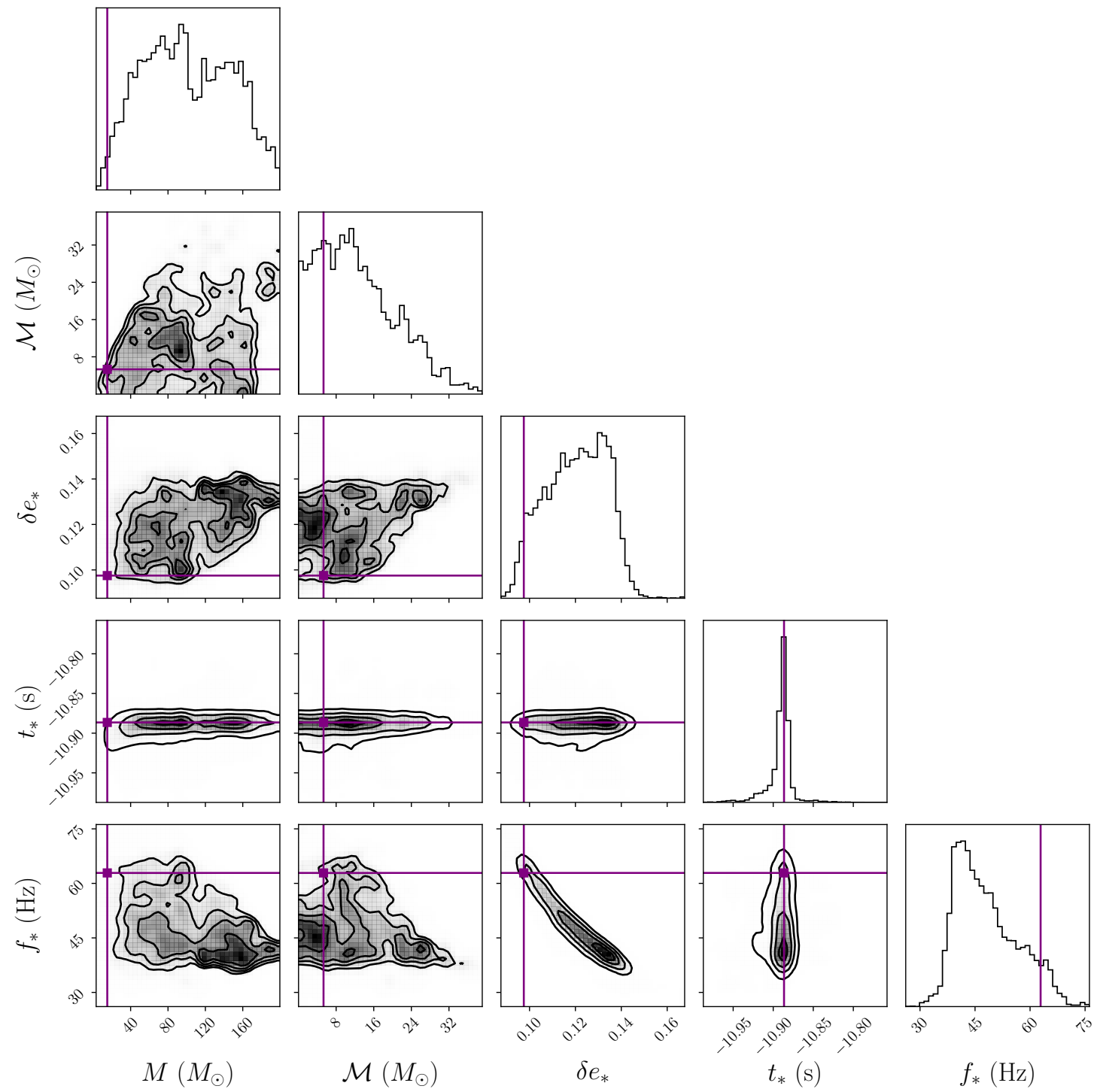

Figure 3.4: Corner plot showing the posterior distributions for the eccentric burst prior meta parameters for a case with SNR per Burst $=4$. This simulation used the highly eccentric waveform model of (East et al., 2013) with signal parameters $M=15 M_{\odot}$ and $\mathcal{M}=5.3 M_{\odot}$. The anchor burst parameters $\left(t_{\star}, f_{\star}, \delta e_{\star}\right)$ are related to the system orbital parameters at the emission of the anchor burst. The time, $t_{\star}$, is measured relative to merger (Cheeseboro \& Baker, 2021). 
isolated near merger. To account for this a change of wavelet basis in the signal model could be implemented. Using a chirplet basis has been shown to improve burst searches for quasi-circular BBHs (Millhouse et al., 2018). A wavelet could be constructed that better represents the high amplitude, high frequency pericenter emission paired with low amplitude, low frequency medial emission. A recent analytic effective flyby waveform has been specifically designed to approximate the GW burst emission from highly eccentric binaries (Loutrel, 2020). Additionally, highly eccentric pericenter emission should be well approximated by the emission from a hyperbolic passage, so the GW model of (Turner, 1977) may provided a useful basis function.

Our method could potentially be applied to data from other GW detectors beyond the current terrestrial interferometers. As binaries are expected to circularize over time (Peters, 1964), accessing lower frequencies and therefore earlier evolution could be a boon for the detection of highly eccentric systems. Third generation terrestrial detectors, which can probe frequencies a factor of a few to 10 lower than the current, second generation detectors, are expected to detect many more systems with non-negligible eccentricity (Lower et al., 2018). Similarly, eccentric sources are expected to be an important source for LISA with a fraction of the population entering the LISA band with very high eccentricity, 1 (Porter \& Sesana, 2010; Samsing \& D'Orazio, 2018; D'Orazio \& Samsing, 2018). The massive black holes that LISA observes will enter the band in some cases years before merger (AmaroSeoane et al., 2017). Because our method is a prior that can be applied to any GW burst search, it could be adapted to work with data from any GW detector suitable 
for a burst search.

This analysis represents a proof of concept for the use of a physically motivated prior to search for highly eccentric BBH systems. We did not perform a comprehensive study for a wide range of simulated sources. As stated in Section 3.3, we generate a small number of sources using different $M$ s. We did not exhaustively explore the parameter space of masses, mass ratios, or eccentricities. A future analysis could consider a more realistic population of sources. This analysis does not use a formal detection statistic. Instead we opt to use a parameter estimation figure of merit as a simple proxy for detection. We choose not to compute the full Bayesian evidence because of the computational expense. A future analysis where our prior is implemented in BayesWave would be able to compute Bayesian evidence ratios for cases with and without the eccentric burst prior.

We believe that using physically motivated priors is a promising method to target GW burst searches towards particular sources. The eccentric burst prior could be implemented as part of a follow-up stage in existing GW burst pipelines. When the pipeline finds a candidate event, this targeted method would search for nearby GW bursts of marginal significance that could have arisen from a highly eccentric binary. Individually these isolated bursts might not stand above the background, but when taken together their significance grows. The code for this work can be found at Baker \& Cheeseboro (2021). 


\subsection{Appendix}

In order to calculate the Jacobian matrices, $J_{+}$and $J_{-}$, of Equation 3.6, we started with the low order series approximations of the centroid mapping equations, Equation 3.3 and Equation 3.4. The two small parameters of the perturbation series are $\delta e=1-e \ll 1$ and $(\pi f \mathcal{M})^{5 / 3} \ll 1$. We analytically computed the derivatives of the centroid mapping equations and truncated the resulting series. Each $J_{ \pm}$matrix was computed as the full $3 \times 3$ matrix which included the effects of $\delta e$ on the centroid 
uncertainty. The nine components of both $J_{+}$and $J_{-}$are presented below.

$$
\begin{aligned}
& J_{+, t t}=\frac{\partial t_{i+1}}{\partial t}=1 \\
& J_{+, t f}=\frac{\partial t_{i+1}}{\partial f}=-\frac{1}{f_{i}^{2}} \sqrt{\frac{2-\delta e_{i}}{\delta e_{i}^{3}}} \\
& J_{+, t \delta e}=\frac{\partial t_{i+1}}{\partial \delta e}=\frac{\delta e_{i}-3}{f_{i} \sqrt{\left(2-\delta e_{i}\right) \delta e_{i}^{5}}} \\
& J_{+, f t}=\frac{\partial f_{i+1}}{\partial t}=0 \\
& J_{+, f f}=\frac{\partial f_{i+1}}{\partial f}=1+\frac{\left(4140+7547 \delta e_{i}\right) \pi}{5402^{2 / 3}}\left(\pi f_{i} \mathcal{M}\right)^{5 / 3}+\frac{3725 \pi^{2}}{1442^{1 / 3}}\left(\pi f_{i} \mathcal{M}\right)^{10 / 3} \\
& J_{+, f \delta e}=\frac{\partial f_{i+1}}{\partial \delta e}=\frac{7547 \pi f_{i}}{5402^{2 / 3}}\left(\pi f_{i} \mathcal{M}\right)^{5 / 3} \\
& J_{+, \delta e t}=\frac{\partial \delta e_{i+1}}{\partial t}=0 \\
& J_{+, \delta e f}=\frac{\partial \delta e_{i+1}}{\partial f}=\frac{17\left(225+121 \delta e_{i}\right) \mathcal{M} \pi^{2}}{812^{2 / 3}}\left(\pi f_{i} \mathcal{M}\right)^{2 / 3} \\
& J_{+, \text {eese }}=\frac{\partial \delta e_{i+1}}{\partial \delta e}=1+\frac{2057 \pi}{1352^{2 / 3}}\left(\pi f_{i} \mathcal{M}\right)^{5 / 3}
\end{aligned}
$$




$$
\begin{aligned}
J_{-, t t} & =\frac{\partial t_{i-1}}{\partial t}=1 \\
J_{-, t f} & =\frac{\partial t_{i-1}}{\partial f}=\frac{1}{f_{i}^{2}} \sqrt{\frac{2-\delta e_{i}}{\delta e_{i}^{3}}} \\
J_{-, t \delta e} & =\frac{\partial t_{i-1}}{\partial \delta e}=\frac{3-\delta e_{i}}{f_{i} \sqrt{\left(2-\delta e_{i}\right) \delta e_{i}^{5}}} \\
J_{-, f t} & =\frac{\partial f_{i-1}}{\partial t}=0 \\
J_{-, f f} & =\frac{\partial f_{i-1}}{\partial f}=1-\frac{\left(4140+7547 \delta e_{i}\right) \pi}{405}\left(\pi f_{i} \mathcal{M}\right)^{5 / 3}+\frac{3725 \pi^{2}}{1442^{1 / 3}}\left(\pi f_{i} \mathcal{M}\right)^{10 / 3} \\
J_{-, f \delta e}= & \frac{\partial f_{i-1}}{\partial \delta e}=-\frac{7547 \pi f_{i}}{5402^{2 / 3}\left(\pi f_{i} \mathcal{M}\right)^{5 / 3}} \\
J_{-, \delta e \delta e}= & \frac{\partial \delta e_{i-1}}{\partial \delta e}=1+\frac{2057 \pi}{1352^{2 / 3}}\left(\pi f_{i} \mathcal{M}\right)^{5 / 3} \\
J_{-, \delta e t} & =\frac{\partial \delta e_{i-1}}{\partial t}=0 \\
J_{-, \delta e f} & =\frac{\partial \delta e_{i-1}}{\partial f}=-\frac{425 \mathcal{M} \pi^{2}}{92^{2 / 3}}\left(\pi f_{i} \mathcal{M}\right)^{2 / 3} \\
&
\end{aligned}
$$




\section{Chapter 4}

\section{Implementing an Eccentricity Search for Pulsar Timing Arrays}

In Chapter 3 we derived an eccentric burst prior that could be implemented into burst search methods like BayesWave to search for highly-eccentric BBHs. While this eccentric burst prior is limited by a few caveats, such as being valid for $e \gtrsim 0.7$ and $r \gtrsim 15 M$, it was still able to recover bursts more efficiently in the intermediate SNR regime (SNR $\sim 3-6$ ) when compared to a uniform prior. With further improvements and more robust testing, it could be implemented into a burst search method. In this chapter we switch to the PTA regime to discuss the work of implementing a search for continuous GWs from an eccentric SMBHB.

\subsection{Introduction}

GWs are now a tool we can use to study our Universe. So far, all detections of GWs have come from high-frequency $(>10 \mathrm{~Hz})$ signals arising from coalescing stellar-mass black holes and neutron stars (e.g. Abbott et al., 2021c). However, as PTAs gradually improve their sensitivity, we come closer to detecting SMBHBs in the form of a GW background and discrete (resolved from the background) signals. Future detections of these individually resolved sources can bring insight into the dynamical evolution of SMBHBs and growth of supermassive black holes, and feedback between binary systems and the broader galactic environment. 
As discussed in Section 1.1.2, SMBHBs are the by-product of galaxy mergers. At large scales, dynamical friction with the broader merger environment of stars and gas helps efficiently centralize a pair, however many different mechanisms have been called upon to drive the binary further: including 3-body interactions with a dense galactic stellar core, torques from a circumbinary disk, other gas-driven fuelling, etc. The dynamical mechanisms required to drive the SMBHBs to $r_{s d} \lesssim 0.1 \mathrm{pc}$, where they emit GWs in the PTA regime, is commonly known as the "last-parsec problem".

Among numerous potential solutions that have been proposed to solve this last parsec problem, a feature of many of them is a prediction that the binaries entering the nanohertz-to-microhertz GW-band may, in fact, be entering as eccentric systems. These conclusions are tentatively supported by constraints on the GW background by pulsar timing that date as far back as 2015; the non-detection of a GW background in past datasets, when compared to predictive modelling for the expected background signal, has indicated that on average non-zero initial binary eccentricities may be occurring (e.g. Shannon et al., 2015; Hazboun et al., 2020).

Soon after a SMBHB enters the pulsar timing band, the binaries are sufficiently close that GWs can take over as the dominant driver for energy loss and binary evolution, and will bring the binary within a few tens of Myr to an efficient coalescence, as described by the orbital evolution in Equation 1.2 and Equation 1.3. It is binaries that enter the pulsar-timing band with eccentric orbits, and that are subsequently driven primarily by GW emission, that are the subject of the work presented here. 
The coupled differential equation that governs binary evolution for an eccentric source in the past was required to be solved numerically. This led to eccentric waveform calculators that proved slow and computationally expensive, making them not ideal to use in broad-scale GW searches (Vogelsberger et al., 2014; Fitts et al., 2017; Zhu et al., 2015, e. g.). Zhu et al. (2015) and Taylor et al. (2016) made critical improvements to search efficiency by using adaptable harmonic searches to require fewer generated waveforms, however those works focused on only using the earthterm, thus do not encompass a full-scope binary search (i. e. they do not account for the usually highly significant evolution that would be seen in the evolutiondelayed pulsar-term; see Section 2.1.2.1 and Figure 2.10). As a result of the heavy computational load requirements, no eccentric SMBHB searches using a full PTA dataset have been performed, although past work has demonstrated techniques on simulated data (Zhu et al., 2015; Taylor et al., 2016). Thus far, only one singlepulsar search has been performed by Jenet et al. (2004) to target the first published model of candidate SMBHB object 3C 66B.

It is worth noting that the above discussion refers to searches for eccentric binaries emitting continuous GWs. Some past work has focused on burst-type searches, as highly eccentric $(e \gtrsim 0.9)$ SMBHBs have rapid amplitude-variations in strain such that they may be sought as burst sources rather than the continuous signals that our methods address (Finn \& Lommen, 2010; Amaro-Seoane et al., 2010).

In this work, we present the implementation and application of the first code able to perform an efficient, full-coherent-PTA search for continuous eccentric GWs. This work is arranged as follows: in Section 4.2, we provide a description of the 
timing data used for our search in addition to the mathematical basis for the search. In Section 4.3 we describe tests of this software on simulated data, in addition to the application of the software to both search for GWs from, and limit, the SMBHB candidate 3C 66B. In Section 4.4 we discuss the implications of this work for more broadly scoped eccentricity searches, in addition to the implications from the limits placed on 3C 66B.

\subsection{Data and Methods}

\subsubsection{Pulsar Timing datasets}

Since its release of its "9-year" data (Demorest et al., 2013)-referring to PTA data with a 9-year total timing baseline - NANOGrav Collaboration has provided a new data release of pulsar time-of-arrival measurements and noise modelling on roughly 1.5-year timescales. The two most recent datasets that were released were the 11-year and 12.5-year datasets (henceforth referred to as N11 and N12 respectively); these names represent the span in the respective datasets for the longesttimed pulsar. The N11 dataset is made up of 45 pulsars. Only 34 of those have time baselines of greater to or equal than 3 years' duration, thus are used for GW searches. A description of the full dataset and timing procedures is provided by Arzoumanian et al. (2018). The N12 dataset is the latest publicly available pulsar timing dataset that includes 47 millisecond pulsars, 45 of which are used for GW searches (Alam et al., 2021). Unlike previous data releases, the N12 data now in-

cludes narrow-band (Alam et al., 2021) and wide-band analysis. For this work, we 
use the narrow-band version of the N12 dataset.

While the N12 dataset provides the best sensitivity due to a longer time baseline, improved pulsar modelling, and other aspects, the N11 dataset was included in this work to provide a direct comparison to the circular-source search sensitivity previously reported for the N11 data (Aggarwal et al., 2019; Arzoumanian et al., $2020 b)$.

\subsubsection{Signal Model}

PTAs are sensitive to fluctuations caused by GWs in the form of timing residuals. Timing residuals without the presence of a GW signal can be represented for each pulsar as follows

$$
\delta t=M \epsilon+n_{w h i t e}+n_{r e d}+s
$$

where $M$ is the design matrix that describes the linearized timing model, and $\epsilon$ is a vector containing the timing model parameter offsets from the best-fit solution. This allows for the timing model, that was fit without the presence of a GW signal, to be adjusted. The $n_{\text {white }}$ and $n_{\text {red }}$ are vectors that contain noise parameters that describe the white and red noise for each pulsar. The vector $s$, represents the residuals due to an induced GW signal.

When a GW passes through the line of sight between us and a pulsar, it induces a signal in the timing residuals, $s$. This signal causes a change in the temporal measurement of the rotational frequency of the pulsar and can be written 
by rewriting Equation 2.5 as

$$
\frac{\Delta \nu\left(t_{E}\right)}{\nu} \equiv z_{\mathrm{GW}}\left(t_{E}\right)=h\left(t_{E}\right)-h\left(t_{P}\right) .
$$

Where $h$ is the dimensionless GW strain, $t_{E}$ is when the GW passes through the solar system barycenter (SSB), $t_{P}$ is when the GW passes through the pulsar, and $\nu$ is the pulsar rotational frequency measured in the SSB frame (Book \& Flanagan, 2011).

The difference of $t_{E}$ and $t_{P}$ can be written as a geometric time delay

$$
\begin{aligned}
t_{P} & =t_{E}-\frac{D_{P}}{c}(1+\hat{n} \cdot \hat{p}) \\
& =t_{E}-\frac{D_{P}}{c}(1-\cos \mu),
\end{aligned}
$$

where $D_{P}$ is the distance to the pulsar and $\hat{p}$ is its vector pointing to the $\mathrm{SSB}, \hat{n}$ is the direction of the observer's line of sight, and $\mu$ represents the angle between $\hat{n}$ and $\hat{p}$. Based on the above equations, one therefore finds that it is the difference between the Earth and pulsar terms, rather than the net integration over the wave in between the Earth and pulsar, that influences the appearance of timing residuals, as seen below. This aspect becomes important particularly for sources with significant frequency evolution, as eccentric sources have. This is because the pulsar term (which encodes the source's strain at some earlier epoch) will differ significantly in frequency and waveform for each pulsar, therefore interferes non-negligibly with the Earth-term waveform.

Using Wahlquist (1987), we can write $h$ with a dependence on the source 
properties, and the relative sky position of the GW source and the pulsar, as

$$
h(t)=\left[\begin{array}{ll}
F_{+} & F_{\times}
\end{array}\right]\left[\begin{array}{cc}
\cos 2 \psi & -\sin 2 \psi \\
\sin 2 \psi & \cos 2 \psi
\end{array}\right]\left[\begin{array}{l}
h_{+}(t) \\
h_{\times}(t)
\end{array}\right],
$$

where $\psi$ is the polarization angle of the $\mathrm{GW}$, and $F_{+, \times}$are antenna pattern functions dependent upon the sky locations of the pulsar and the GW source. (A full description of $F_{+, \times}$can be found in Sesana \& Vecchio 2010; Ellis et al. 2012; Taylor et al. 2016).

The passing GW induces a redshift in the expected timing of a pulse from a pulsar in its residuals. This redshift is given by

$$
R\left(t_{E}\right)=\int_{0}^{t_{E}} z_{\mathrm{GW}}\left(t^{\prime}\right) d t^{\prime}=s\left(t_{E}\right)-s\left(t_{P}\right),
$$

where $s(t)$, as in Equation 4.1, is the expected signal at that pulsar, constructed as

$$
s(t)=\int_{0}^{t} h\left(t^{\prime}\right) d t^{\prime}=F_{+} s_{+}+F_{\times} s_{\times} .
$$

With $h(t)$ as defined in Equation 4.4, we get

$$
s_{+, \times}=\int_{0}^{t} h_{+, \times}\left(t^{\prime}\right) d t^{\prime}
$$

Finally, we must add in the model for the GW signal emitted from the eccentric binary. The GW strain produced by a non-spinning, eccentric SMBHB source for 
both polarizations scales as

$$
\begin{aligned}
h_{+} & \sim \frac{G M \eta}{D_{L} c^{2}} \chi \frac{1}{(1-\chi)^{2}} h_{+}\left(e_{t}, i, \phi, u\right), \\
h_{\times} & \sim \frac{G M \eta}{D_{L} c^{2}} \chi \frac{2 c_{i}}{(1-\chi)^{2}} h_{\times}\left(e_{t}, i, \phi, u\right),
\end{aligned}
$$

where $D_{L}$ is the luminosity distance, $e_{t}$ is the parameterized "time" orbital eccentricity that is associated with the $\mathrm{PN}$-accurate Kepler equation (Memmesheimer et al., 2004), $M=m_{1}+m_{2}$ is the total mass, $\eta=\frac{m_{1} m_{2}}{M^{2}}$ is the symmetric mass ratio, $i$ is the inclination, $\phi$ is the orbital phase, and $u$ is the eccentric anomaly. The dimensionless PN parameter, $\chi=\left(\frac{G M n}{c^{3}}\right)^{2 / 3}$, where $n$ comes from the Kepler equation and is related to the orbital period by $n=\frac{2 \pi}{P_{b}}$. For a full description of $h_{+, \times}$as formulated above, please refer to Boetzel et al. (2017).

As demonstrated by Susobhanan et al. (2020), a sufficiently accurate calculation of the orbital evolution driven by GW radiation for a non-spinning black holes in precessing eccentric orbits requires computing $R(t)$ (Equation 4.5) for a source evolving in a complex way. The novelty introduced by Susobhanan et al. (2020) is the semi-analytic approach to determine $n(t), e_{t}(t), \gamma(t)$, and $l(t)$ from $n\left(e_{t}\right)$ and $t\left(e_{t}\right)$ (See Section II. C of Susobhanan et al. (2020) for the full description). This semi-analytic approach has proven to be more computationally efficient than a fully analytic/numerical approach, making it possible to be implemented into an eccentric GW search. 


\subsubsection{Software}

For this study, we integrate and augment three codes to allow an eccentric GW search. We make use of enterprise (Ellis et al., 2020), which is a GW and pulsar timing analysis software suite; PTMCMCsampler (Ellis \& van Haasteren, 2017), which is an ensemble sampler, and a modified version of GWecc (Susobhanan, 2020; Susobhanan et al., 2020), which codifies the production of pulsar timing residuals induced by a GW from a non-spinning, precessing, eccentric SMBHB. This code was based on the semi-analytic model for GW eccentricity evolution by Susobhanan et al. (2020) that was described in the previous section.

Our treatment of the reading and processing of pulsar timing residuals was handled by enterprise and was therefore built from the same basis used in Aggarwal et al. (2019) and Arzoumanian et al. (2020b), and we refer readers particularly to Aggarwal et al. (2019) to rigorously understand handling of pulsar timing data. In brief, enterprise has codified structures for pulsar time-of-arrival data and noise models, such that it can build the residuals we represented above by Equation 4.1. The simulation of accurate timing residuals based on eccentric SMBHBs is central to the injection-and-recovery analysis and search methods we describe below. To improve compatibility between GWecc and enterprise, we developed a pythonbased version of GWecc, ensuring the correct call-and-response formatting between the two codes. Thus, our python-based version of GWecc allows us to simulate GW signals from eccentric binaries, relying on the semi-analytic approach of Susobhanan et al. (2020) to approximate the evolution required to accurately model the pulsar- 
term signal for a binary source (Equation 4.3).

\subsubsection{Creation of the Simulated Dataset}

To test the functionality of GWecc in enterprise, we performed analysis to inject and recover an eccentric waveform. For this analysis, we generated simulated pulsar timing residual data using the N12 dataset. We utilized libstempo (Vallisneri et al., 2013) which is a python-wrapped version of TEMP02 (Hobbs et al., 2006), a pulsar-timing analysis software package.

For each of the pulsars, we removed all sources of noise and other artifacts that are present in their timing residuals, forcing them to zero. This process makes the residuals "ideal" so that we can inject known signals to recover in the injection analysis. We then generate GW-induced residuals, including Earth- and pulsar-term signals that were evolved using GWecc. This defined a model SMBHB with a set of orbital parameters. The reference epoch, $t_{0}$, is usually set to the last TOA of the dataset. This is important for the calculation of $\tau_{0}$, a dimensionless time parameter in GWecc, as it checks if the selected binary parameters satisfy $\left(t-t_{0}\right)<t_{\text {merge }}$. This condition is satisfied when using the last TOA in the dataset. For our injected signals, we selected parameters that ensured a bright enough signal to be securely detected (e.g. large mass, relatively nearby). That signal $s$ was injected into the residuals. Upon injection of the eccentric GW signal, we also inject back the white and red noise for each pulsar. This makes the simulated data as close to the real data as much as possible. Once the signal has been generated, we use libstempo 
to save the injected signal and noise as a new pulsar residual.

\subsubsection{Procedure for Detecting and Limiting GWs}

To carry out a search for a GW in a dataset, we use enterprise to create what is called a PTA object, which stores all of the information on the pulsars in our array and incorporates them into our signal model that is defined in Section 4.2.2.

For our proof of concept we limit the scope of this work to apply a search for a system at a specific initial orbital frequency $F_{\text {orb }}$ (statistically, this is equivalent to testing for the presence of a binary at a particular orbit at specific reference epoch, for instance as applied later to 3C66B. In terms of the code, this implies we are either holding constant, or placing a delta-function prior, on the orbital frequency parameter). If no signal is obtained from the data, then we aim to place limits on the chirp mass of the binary candidate. Due to a significant added complexity that is beyond the scope of this work, we leave a search over the full PTA frequency range for future efforts.

For this analysis, we set uniform priors on $\log _{10}(\mathcal{M}), e_{0}, q, \phi_{p}, l_{0}$, and $\gamma_{p}$; here, $\log _{10}(\mathcal{M})$ is the base 10 logarithm of the chirp mass, $e_{0}$ is the initial orbital eccentricity, $q=\frac{m_{2}}{m_{1}}$ is the mass ratio with the convention $\frac{m_{2}}{m_{1}} \leq 1, l_{0}$ is the mean anomaly, $\phi_{p}$ is the orbital phase in the pulsar's frame, $\gamma_{p}$ is the angle of periastron in the pulsar's frame, and $\psi$ is the GW polarization angle. For $F_{\text {orb }}, i$, and the source distance $D_{L}$, we hold them fixed at a constant value. All of these parameters are defined at the reference epoch, $t_{0}$, which is defined as the last TOA in the dataset. 


\begin{tabular}{|c|c|c|}
\hline Parameter & $\begin{array}{l}\text { Uniform } \\
\text { min }\end{array}$ & $\begin{array}{l}\text { Prior Ranges } \\
\max \end{array}$ \\
\hline $\log _{10}(\mathcal{M})$ & 7 & 11 \\
\hline$e_{0}$ & 0.001 & 0.99 \\
\hline$q$ & 0.1 & 1 \\
\hline$\phi_{\mathrm{P}}$ & 0 & $2 \pi$ \\
\hline$l_{0}$ & 0 & $2 \pi$ \\
\hline$\gamma_{\mathrm{P}}$ & 0 & $2 \pi$ \\
\hline$\gamma_{0}$ & 0 & $2 \pi$ \\
\hline$\psi$ & 0 & $2 \pi$ \\
\hline
\end{tabular}

Table 4.1: Here we list the search parameters and their uniform prior ranges that were utilized for searches done on the 11-year and 12.5-year NANOGrav datasets.

For the pulsar distances, $D_{p}$, we adopt a normal distribution prior centered on a mean value and error determined by parallax measurements, or to $D_{p}=1 \pm$ $0.2 \mathrm{kpc}$ if the distance for a pulsar is unknown (e.g. Arzoumanian et al., 2020b). Typically pulsar distances are not well known (even to a few kpc accuracy), except for the nearest pulsars that may be measured via parallax using very long baseline interferometry, or by fitting for parallax in the timing data itself. Thus, for many pulsars, the error range on their distances can be broad. Hence why we assume $D_{p}=1 \pm 0.2 \mathrm{kpc}$ for pulsars with unknown distances. Searching over $D_{p}$ and $\phi_{p}$ separately allows us to break the degeneracy between the two parameters, making it easier to sample the parameter space. As the prior is returned in all cases, our searches do not inform significantly on $D_{p}$.

We also search over the power spectral density of each pulsar's intrinsic red noise which is modeled as follows

$$
P=\frac{A_{\text {red }}^{2}}{12 \pi^{2}}\left(\frac{f}{f_{\text {year }}}\right)^{\gamma} \mathrm{yr}^{3}
$$


by allowing the red noise amplitude $A_{\text {red }}$ and power-law red-noise spectral index $\gamma$ to vary in our MCMC simulation. As red noise can vary from pulsar to pulsar, we also include the red noise empirical distributions. These distributions are utilized by the jump proposals, which in turn, informs our search about the parameter space. These red noise empirical distributions were created from single pulsar run noise posterior distributions. White noise effects are fit for using three parameters called "EFAC", "EQUAD", and "ECORR". EFAC is a multiplicative factor that effects the uncertainties on each TOA, EQUAD simply adds the error from each known noise source in quadrature, and ECORR mainly fits for random correlated noise (i.e. jitter; see Section 5.6 of Lam (2016) for further reading.) Red noise is also important to account for, since it can closely resemble the noise properties of the stochastic GW background (Hazboun et al., 2020). Numerous models have been developed to help characterize these noise sources (Breivik et al., 2016; Lam et al., 2018; Madison et al., 2019).

While a common red noise process is not expected to have an impact at higher GW frequencies, we still incorporated it into our model by holding it fixed at $\log _{10}\left(A_{\mathrm{GW}}\right)=-15.696$ and $\gamma_{\mathrm{GW}}=6.23$. These values were determined from the maximum likelihood values that were calculated from GW stochastic background searches over the N12 dataset.

Once all the components of the signal model (See Section 4.2.2) have been established, it is then applied to pulsars in our dataset. As the parameter space is quite large, we make use of jump proposals to ensure that each parameter's prior is being sampled to better inform the walker as it moves about the parameter space, 
and also group covariant parameters together for ease of sampling. From there we then run an MCMC to collect posterior samples.

The search procedure on a simulated vs real dataset is essentially the same. For the simulated datasets created as described in Section 4.2.4 we don't include a fixed common red noise process into our model as the only noise sources we incorporate are the white and intrinsic red noise for each pulsar.

\subsubsection{Detection Statistic}

To determine if our search resulted in a detection or a non-detection, we rely on the Savage-Dickey Bayes factor relation (Dickey, 1971)

$$
\mathcal{B}_{10} \equiv \frac{\text { evidence }\left[\mathcal{H}_{1}\right]}{\text { evidence }\left[\mathcal{H}_{0}\right]}=\frac{p\left(h_{0}=0 \mid \mathcal{H}_{1}\right)}{p\left(h_{0}=0 \mid \mathcal{D}, \mathcal{H}_{1}\right)}
$$

where $p\left(h_{0}=0 \mid \mathcal{H}_{1}\right)$ is the prior probability of $h_{0}=0$ in the model $\mathcal{H}_{1}$ and $p\left(h_{0}=\right.$ $\left.0 \mid \mathcal{D}, \mathcal{H}_{1}\right)$ is the posterior probability of $h_{0}=0$ in the model $\mathcal{H}_{0}$. $\mathcal{H}_{1}$ represents a signal-plus-noise model model whereas $\mathcal{H}_{0}$ represents a noise-only model. This relation works well for our setup since $\mathcal{H}_{0}=\mathcal{H}_{1}$ when $h_{0}=0$ making these models nested. For our search we simply calculate the Bayes factor from the posterior samples of $\log _{10}(\mathcal{M})$. The error in the Bayes factor is calculated as

$$
\sigma=\frac{\mathcal{B}_{10}}{\sqrt{n}}
$$

where $n$ is the number of samples in the last amplitude bin. 


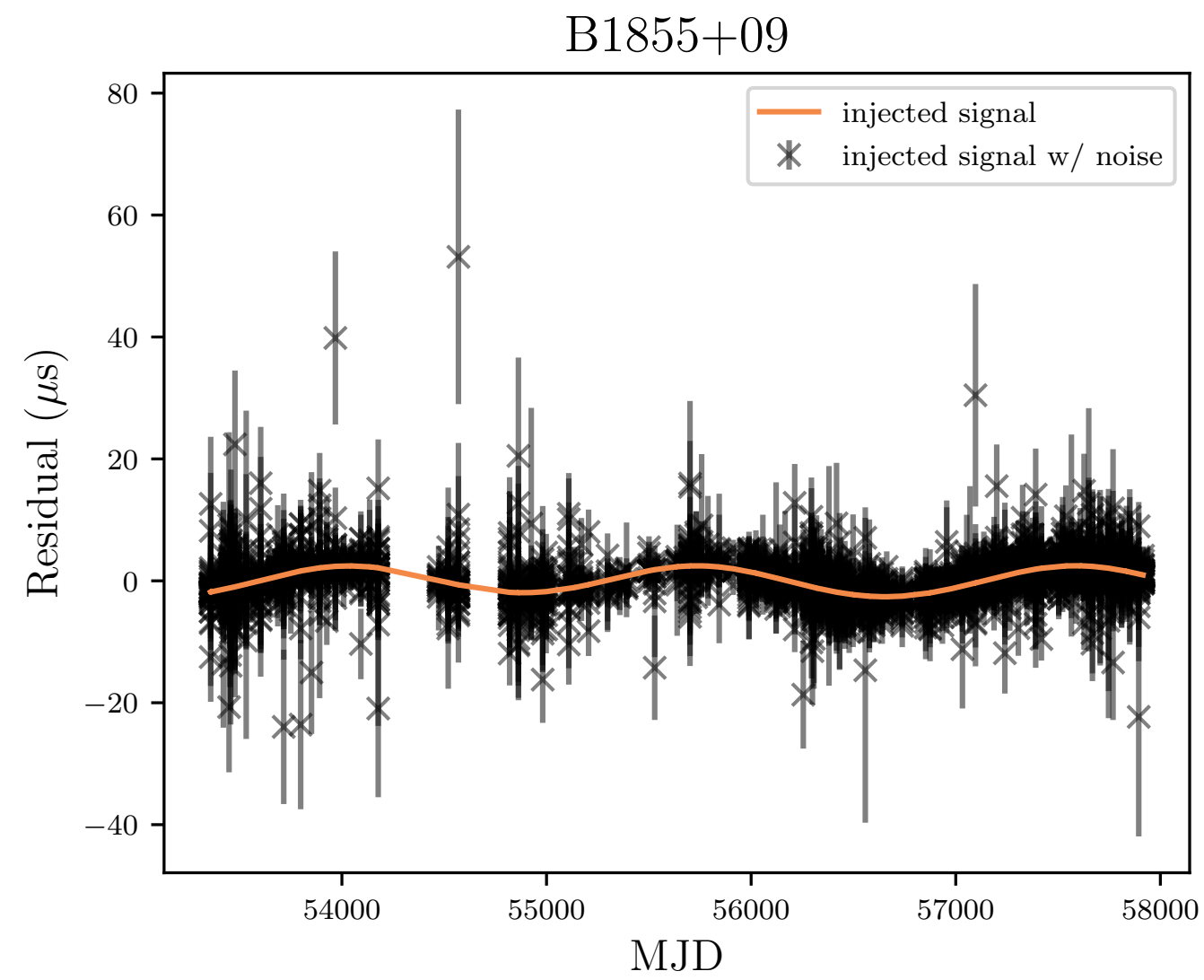

Figure 4.1: A simulated waveform and timing residual, including the Earth and pulsar term, as it would appear in PSR B1855+09. The black data-points are the TOAs with the injected signal and noise. The orange line is the injected signal as described in Section 4.2.4 with parameters given in Table 4.2

If a signal is present in the dataset, then we expect the posterior distributions to deviate from the parameter priors. This is further confirmed if the Bayes factor value is $\mathcal{B}_{10}>100$, signifying strong evidence for the presence of an eccentric GW. If a signal is not present in the dataset, then we expect the posterior distributions to be prior-dominated. Meaning that they are essentially indistinguishable from the parameter priors. This will also result in a Bayes factor of $\mathcal{B}_{10} \leq 1$, signifying no evidence of an eccentric GW signal being present in the dataset. 

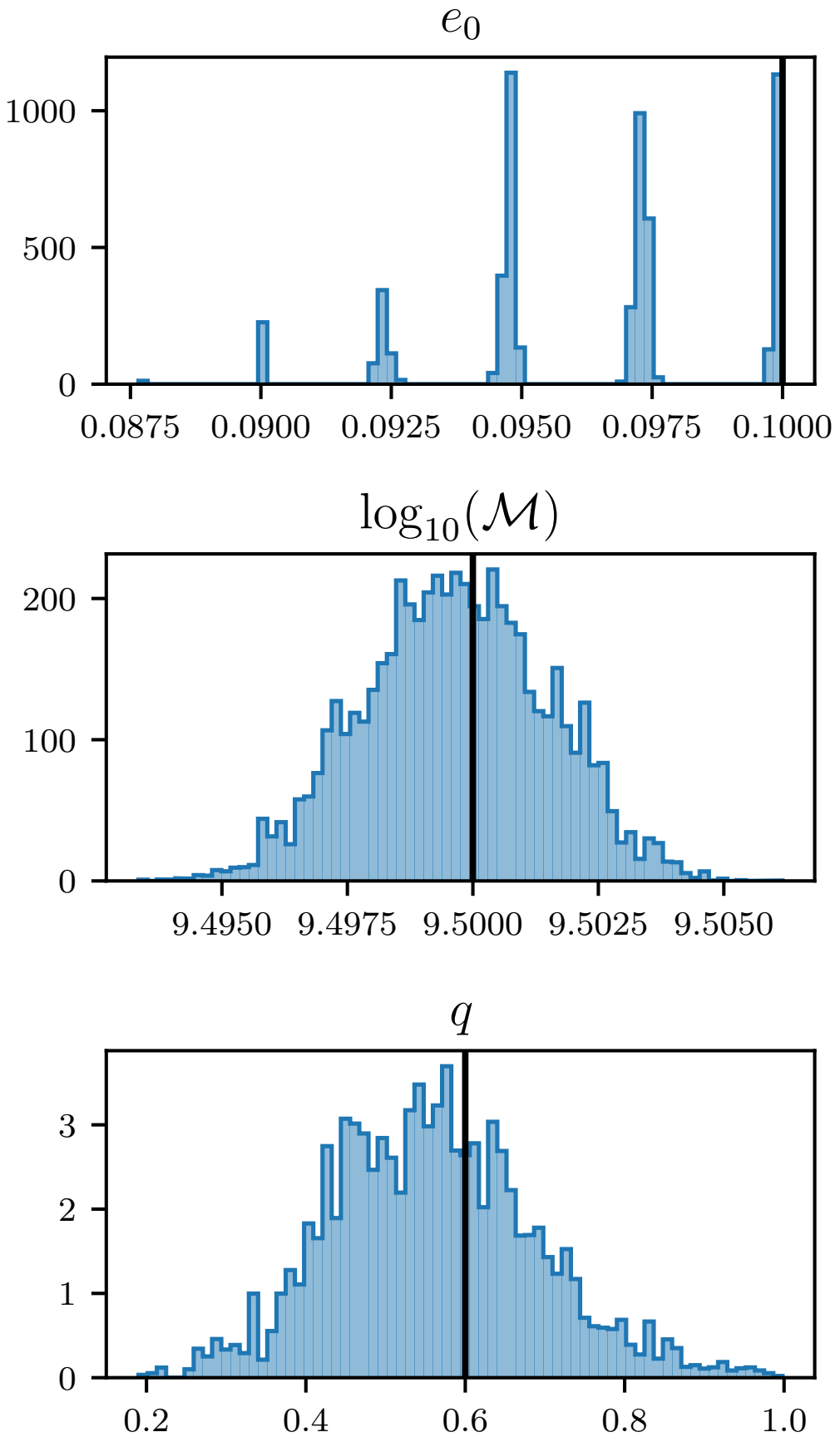

Figure 4.2: Posterior distributions for global search parameters from our simulated injection analysis (Sections 4.2.4,4.3.1). The top panel shows the initial eccentricity, the second panel shows base-10 log of chirp mass, and the bottom panel shows the mass ratio. Black lines indicate the injected values in the relevant parameters. The priors used on the initial eccentricity and the base-10 log of chirp mass were restricted to a smaller range, but resulted in poor sampling of the initial eccentricity which can be seen in its posterior distribution as a "comb-like" structure. 


\subsection{Results and Discussion}

\subsubsection{Injection Test Results}

To demonstrate the basic simulation and search functionality of this code, we performed a signal injection and recovery for a bright source, as described in Section 4.2.4. This source was assumed to be at a fixed sky position and initial orbital period. We chose a particular eccentricity, mass, mass ratio, and distance values to ensure we injected a source that would 1) be detected and 2) not experience evolution of more than one period bin within the timespan of the data.

The priors we used for this search have distributions consistent with those described in Section 4.2.5 (flat priors for most parameters), but for this test we held $l_{0}, \gamma_{0}$, and $\psi$ fixed at 0 . As for $\log _{10}(\mathcal{M})$ and $e_{O}$, we restricted their prior ranges to $9.4<\log _{10}(\mathcal{M})<9.6$ and $0.001<e_{O}<0.1$ respectively. This is because we had issues recovering the signal at the full prior ranges stated in Table 4.1. The prior on $q$ remained the same as stated in Table 4.1. We also didn't incorporate a fixed common red noise process for this injection.

The injected signal values are presented Table 4.2, and a visualization of the waveform and injected residuals for this source (for simulated data from NANOGrav pulsar B1855+09) are shown in Figure 4.1 for reference. Figure 4.2 shows the resulting example of an accurate signal recovery for a simulated signal injected into the data. The Bayes factor for this detection is $\mathcal{B}_{10}=420 \pm 88$, indicating a confidently detected signal.

The range of parameters recovered is relatively narrow for chirp mass, is less 


\begin{tabular}{cc}
\hline & Injected Values \\
\hline $\mathrm{RA}_{\mathrm{GW}}(\mathrm{rad})$ & 5.01 \\
$\mathrm{DEC}_{\mathrm{GW}}(\mathrm{rad})$ & 1.96 \\
$\log _{10}\left(D_{L}\right)(\mathrm{pc})$ & 7.5 \\
$\log _{10}(\mathcal{M})$ & 9.5 \\
$e_{0}$ & 0.1 \\
$q$ & 0.6 \\
$i(\mathrm{rad})$ & $\pi / 3$ \\
$\phi_{\mathrm{P}}(\mathrm{rad})$ & 0 \\
$l_{0}(\mathrm{rad})$ & 0 \\
$\gamma_{\mathrm{P}}(\mathrm{rad})$ & 0 \\
$\gamma_{0}(\mathrm{rad})$ & 0 \\
$\psi(\mathrm{rad})$ & 0 \\
\hline
\end{tabular}

Table 4.2: List of injected parameter values utilized in the injection analysis described in Section 4.3.1.

constrained for mass ratio, and appears as a comb function for eccentricity. The origin of this comb function is not clear after investigation, but is likely an artifact of poor sampling at this chirp mass value. We also attempted an injection-recovery study using the full prior ranges detailed in Table 4.1 and had difficulty recovering the injected signal.

\subsubsection{Application of the Eccentric Search Method on Real Data}

Here, we demonstrate the application of this software to a full pulsar dataset and note some special considerations and limitations of the search when doing so. 


\subsubsection{An Illustrative Example of Code Considerations: Binary Can- didate 3C 66B}

To apply the data to a full pulsar dataset, for the purposes of illustrating the application of a targeted search, we opt to target a 3C 66B-like source. 3C 66B is an elliptical galaxy that was originally reported as a SMBHB candidate by Sudou et al. (2003) based on elliptical motion of the radio core; this motion was interpreted to be the result of orbit-modified precession of a jet from one of the SMBHs. Those observations were fit with an observed orbital period of $1.05 \pm 0.03$ yr (i.e. a restframe orbital period of $\sim 1.07 \mathrm{yr}$ considering the cosmological redshift of $z=0.0215$ for 3C 66B). Sudou et al. (2003) originally proposed a binary model with a maximum chirp mass $\mathcal{M} \sim 10^{10} M_{\odot}$, however individual constituent masses and detailed orbital modelling were not performed in that work. There have been many subsequent radio observations of the core of this source (e.g. Zhao et al., 2011, 2015; Sudou et al., 2017), however none with astrometric precision sufficient to confirm the sub-milliarcsecond ellipses originally detected by Sudou et al. (2003). Iguchi et al. (2010) subsequently detected a $93 \pm 1$ day flux variability at $3 \mathrm{~mm}$ wavelength, which can be modelled as a Doppler-shifted modulation effect that shows consistency with an SMBHB of $\sim 1.07$ year rest-frame orbit. The latter work, Iguchi et al. (2010), re-fit the orbital parameters of the system based on their modelled Doppler modulation, assuming a circular orbit and finding a model fit by masses $m_{1}=1.2_{-0.2}^{+0.5} \times 10^{9} \mathrm{M}_{\odot}$, and $m_{2}=7.0_{-6.4}^{+4.7} \times 10^{8} \mathrm{M}_{\odot}$.

Pulsar timing observations have previously provided meaningful constraints on 
this system as a binary, including the limits placed on mass and eccentricity using a single pulsar by Jenet et al. (2004), the broader statistical limits (based on the non-detection of a GW background) arguing for the statistically anomalous nature of an Iguchi-like model from Zhu et al. (2019), and the direct constraints placed on the chirp mass of a circular system by Arzoumanian et al. (2020b).

The case of this source is an interesting one to consider when discussing targeted searches for eccentric GW sources. In particular, it is worth considering that the decay (or inspiral) timescale of a binary whose inspiral is driven by only GW emission is given by:

$$
t_{\mathrm{gr}}=\frac{5}{256} \frac{c^{5} a^{4}}{G^{3} \mu M^{2}} F(e)^{-1}
$$

(Peters, 1964), where $F(e)$ is as given by Equation 1.5. For convenience we use Kepler's third law to cast this expression into a function of rest-frame orbital period, and in terms of chirp mass:

$$
t_{\mathrm{gr}}=\frac{5 c^{5}}{256(2 \pi)^{8 / 3} G^{5 / 3}} T_{\mathrm{orb}}^{4 / 3} \mathcal{M}^{-5 / 3} F(e)^{-1}
$$

Recall that $F(e)$ increases with higher eccentricity; $F(0)=1$, growing gradually to $F(0.6)=10, F(0.8) \simeq 100$, and increasing to infinity at $F(e \rightarrow \infty)$. Therefore, particularly at higher eccentricities, the inspiral timescale of a binary can be significantly shorter than if it were circular. As shown in numerous past works (Lee et al., 2011, e. g.), the bulk of SMBHB sources in circular orbits will be detected as sources that to not evolve appreciably (that is, they stay within one frequency bin) for the 
entire duration of the pulsar data set. However, because of the more rapid orbital evolution that an eccentric source undergoes (due to e.g. the effect that Equation 1.5 has on boosting the emitted power), particularly at high masses, sources can evolve significantly or even coalesce within the time frame of a years-to-decades-long pulsar data set. Therefore, PTAs have decreased sensitivity not only at the low-mass end (due to intrinsic noise in the detector), but also at the high-mass, high-eccentricity end (due to a lack of signal emitted in the PTA band).

Considering 3C 66B, the reference epoch reported by Sudou et al. (2003) for our initial orbital period was 2003. However, for binaries of significant mass, the orbital evolution for even a circular source should have been sufficiently rapid so as to merge the system before or during the N12 data span. The coalesence year as a function of chirp mass for a $3 \mathrm{C} 66 \mathrm{~B}$-like system is shown in Figure 4.3. When Arzoumanian et al. (2020b) treated this source, they noted this effect could exist at the highest masses, however decided that because the intrinsic senstivity of the N11 data set was at least one order of magnitude below those highest masses, that it did not need to be taken into account.

In eccentric GW searches, this effect is more prominent when compared to current PTA sensitivities and can complicate how we carry out runs and report PTA sensitivity to eccentric GWs. For instance, let us assume a reference epoch of 2003 for an source-frame orbital period of 1.07 years. Figure 4.3 shows the combination of masses and eccentricities that will coalesce before the end of the N11 and N12 NANOGrav data sets; note that the longer data set is affected at a smaller mass range, but only negligibly. Thus, we can consider that above the lines drawn, the 


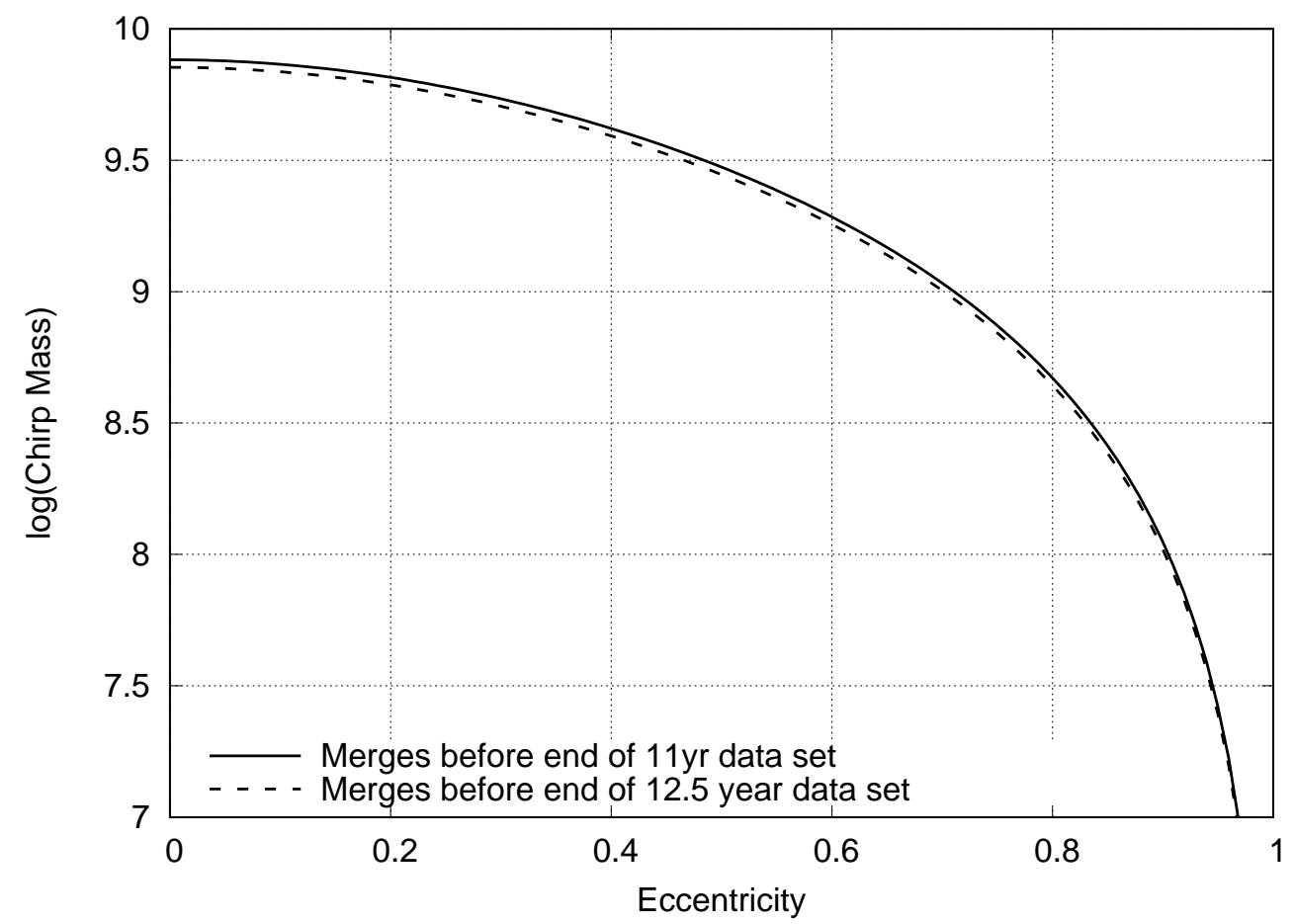

Figure 4.3: This figure represents specifically the source 3C 66B, where the restframe orbital period of 1.07 years is placed at a reference epoch of Gregorian calendar year 2003. For systems at or above the lines shown, a binary of the given chirp mass and eccentricity will merge before the end of the NANOGrav data sets searched in this paper. 
sensitivity of these NANOGrav data sets to 3C $66 \mathrm{~B}$ diminishes rapidly because of the lack of signal entering the data.

We can compare Figure 4.3 to the chirp-mass sensitivity of NANOGrav's N11 data based on the results of Arzoumanian et al. 2020b. If we assume for the sake of this argument that NANOGrav is approximately sensitive to systems of $\log \left(M_{c} / M_{\odot}\right) \gtrsim 9.0$ at all eccentricities, we see that both NANOGrav datasets are completely insensitive to 3C 66B at eccentricities above $e \sim 0.65$.

Thus, it can be clear that the orbital period, period reference epoch, chirp mass, and eccentricity should all be taken into account when performing a search of eccentric sources (and particularly, these limitations should be provided for special consideration when discussing the results of blind searches for GWs). As first noted in Section 4.2.4, the parametric time parameter, $\tau$, essentially encodes the merger time. For GWecc, the evolution equations break down as $\tau \rightarrow 0$. To mitigate this, it is crucial to set $t_{0}$ at the last TOA of the dataset to satisfy the condition $\left(t-t_{0}\right) \ll t_{\text {merge }}$. This is also standard in circular searches (e.g. Arzoumanian et al., $2020 b)$.

The implication of this decision is the following: while this reference-epoch standard can be (though is not always, particularly for the highest frequencies) the right decision for a circular-source search, for our 3C 66B search we are essentially limiting ourselves to searches that are valid only below an eccentricity at which there is little source evolution between the actual binary's reference epoch and the PTAend reference epoch. For us, noting (based on the sections below) that our limits appear to be eccentricity-independent at a $\log (\mathcal{M})$ of around 9.0, this means we are 
limiting our sensitivity to the actual 3C 66B to eccentricities below, conservatively, around $e<0.6$. Again, it is sources below that eccentricity that have insignificant evolution between 2003 and now assuming the source has masses within around 0.2 dex of the mass limit.

\subsubsection{GW search for a 3C 66B-Like Source}

In spite of the caveats in the previous section, it is a useful exercise to perform a search for a "3C 66B-like" source in NANOGrav data to scope the computational complexity and application of our method on real-data and compare with the sensitivity and results from previous searches. In our search, the source is assumed to have a 1.05-year period at the epoch of the last TOA in the dataset (57388 for the N11 data, 57933 for the 12-year data), while for the actual source the reference epoch is the years 51910-52275 MJD. This amounts to a 20-year difference, which will have significant impact on the results based on the rapid evolution of such a source at high mass and eccentricity. This is why we label our target source as 3C 66B-like to reflect this discrepancy. An example 15-year waveform for a 3C 66B-like source at two different eccentricities is shown in Figure 4.4.

To apply a targeted source for a 3C66B-like system in our data, we use a representative luminosity distance of $85 \mathrm{Mpc}$ to the source (Sudou et al., 2003), a seed Earth-frame orbital period of $1.05 \mathrm{yr}$, and a position equal to that of $3 \mathrm{C} 66 \mathrm{~B}$. We place no constraints on the other orbital elements noted in Section 4.2.5, instead leaving them as global search parameters. 

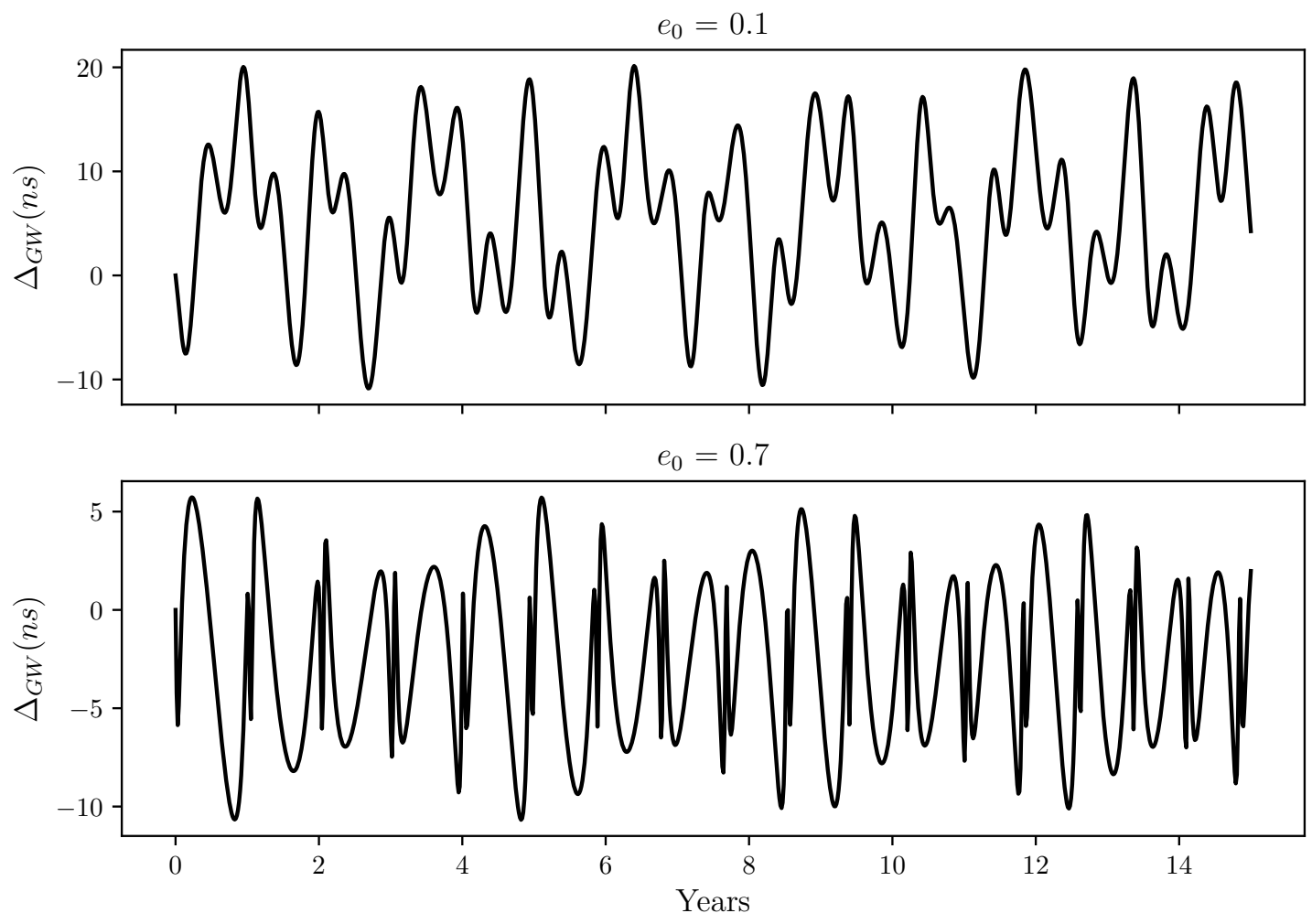

Figure 4.4: Here we show the expected contribution to the timing residual of PSR J1909-3744 expected from 3C 66B at different eccentricities. The sum of Earth and pulsar term are shown in both cases. We assumed parameters $\mathcal{M}=7.9 \times 10^{8} M_{\odot}$, and $q=0.58$. This system was assumed to be face-on.

We applied the detection procedure as described in Section 4.2.5 on both the NANOGrav 11 and 12.5 year datasets, ranging our priors as reported in Section 4.2.5 and Table 4.1.

We also found that, for similar reasons discussed in Arzoumanian et al. (2020b), the inclusion of PSR J1713+0747 resulted in poor sampling as the sampler would utilize J1713+0747 to hide excess power in its intrinsic red noise amplitude. Therefore we opted to remove J1713+0747 from both the N11 and N12 datasets for runs on this 3C 66B-like system.

Due to poor sampling, some samples were removed from the posterior dis- 
tributions by dividing the chain into smaller chunks, calculating the range of those chunks, and comparing each chunk to the range of the full chain. If the range $e_{\text {chunk }}<$ $\left(0.15 \times\right.$ range $\left._{\text {chain }}\right)$, then those samples were removed to minimize the effects of poor sampling. The resulting posterior distributions for chirp mass and initial eccentricity for each dataset can be seen in Figure 4.6 and Figure 4.5 for the N11 and N12 datasets, respectively. The resultant distributions appear to represent nondetections based on their lack of sample build-up at a particular value for these parameters; this is supported by the lack of Bayesian evidence for a detection. The calculated Bayes factors (See Section 4.2.6) for both the N11 and N12 runs are $\mathcal{B}_{11}=0.3 \pm 0.1$ and $\mathcal{B}_{12.5}=0.3 \pm 0.1$, respectively, indicating a non-detection of a 3C 66B-like source at all mass and eccentricity values in both datasets.

To calculate the limits for $\log _{10}(\mathcal{M})$ as a function of $e_{0}$, it is customary to do an upper-limit enterprise run. This type of run utilizes a linear exponential prior that can determine the limit without depending on the prior bounds. We attempted to utilize this type of run but experience poor sampling. Therefore we opted to reweight the posterior samples of $\log _{10}(\mathcal{M})$ into $\log$-space to calculate the limit. First we binned the $\log _{10}(\mathcal{M})$ posterior samples in increments of 0.1 in eccentricity. Then for each bin we used the python package wquantiles (Sabater, 2015) to calculate the 95\% upper limit. As the allowed chirp masses and eccentricities are co-variant, our previously noted sampling biases are eccentricity dependent. To calculate the error on the $95 \%$ upper limit values we incorporated a bootstrap method. This method resamples the posterior distributions in each eccentricity bin and recalculates the $95 \%$ upper limit value for $N$ iterations where we set $N=1000$. We then take the 

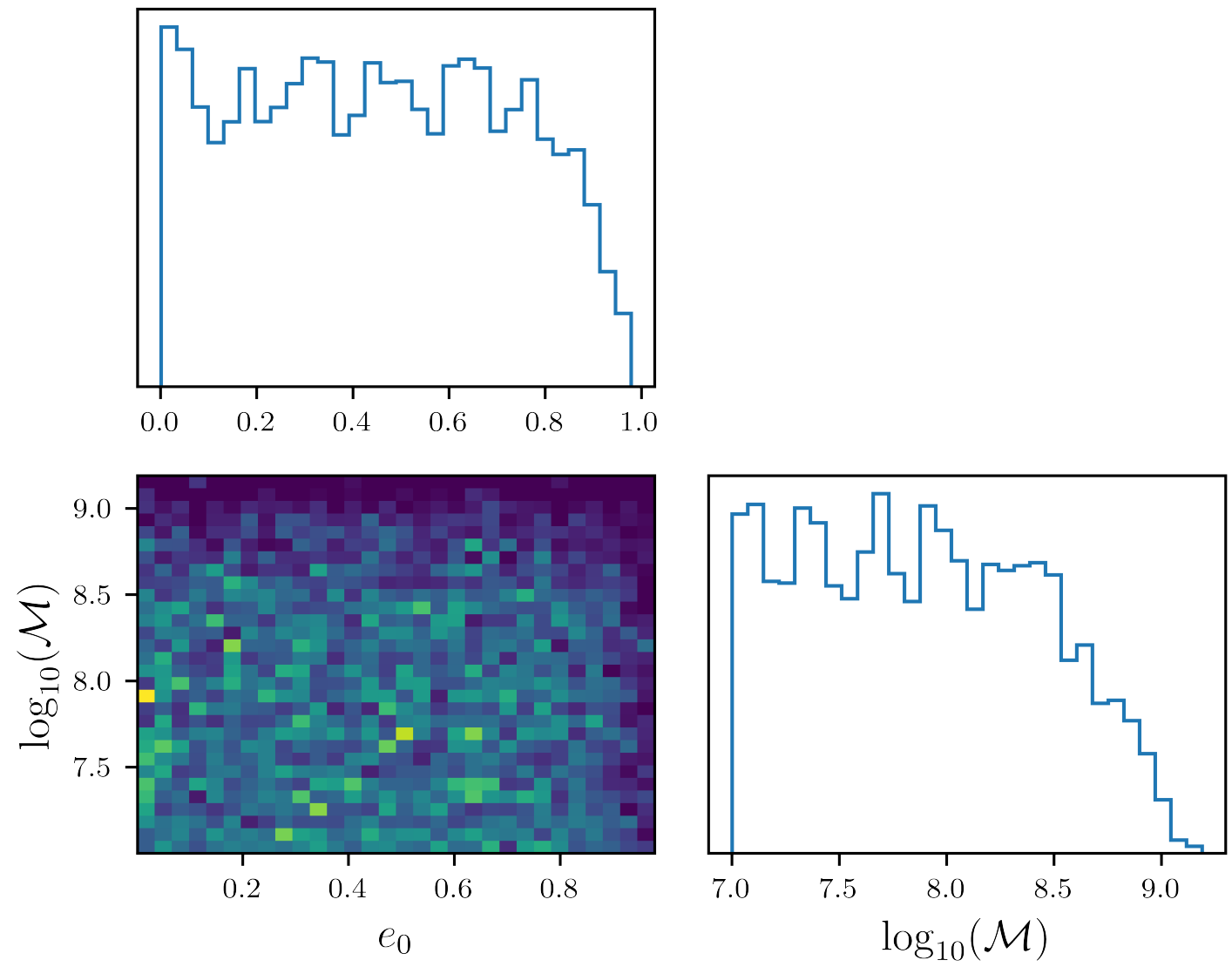

Figure 4.5: Corner plot showing 1-D and 2-D distributions for $e_{0}$ and $\log _{10}(\mathcal{M})$ using the posterior samples obtained from the analysis done on the NANOGrav 12.5-year dataset. 

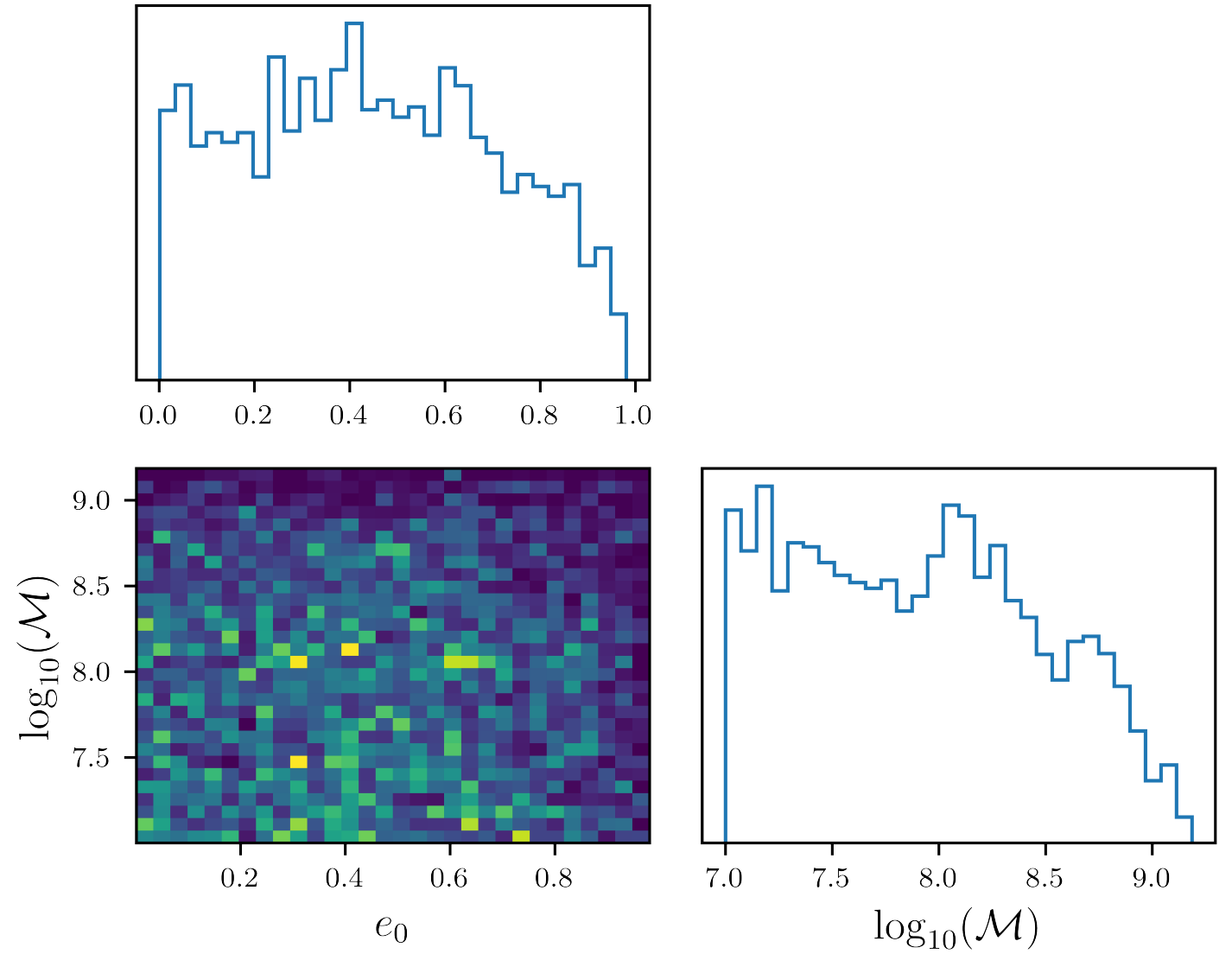

Figure 4.6: Corner plot showing 1-D and 2-D distributions for $e_{0}$ and $\log _{10}(\mathcal{M})$ using the posterior samples from the analysis on the NANOGrav 11-year dataset. 


\begin{tabular}{ccc}
\hline & \multicolumn{2}{c}{$\mathbf{9 5 \%} \log _{\mathbf{1 0}}(\mathcal{M})$ limit } \\
$\mathbf{e}_{\mathbf{0}}$ & 11-year & $\mathbf{1 2 . 5}$-year \\
\hline $0.0-0.1$ & $9.043 \pm 0.007$ & $9.0378 \pm 0.0007$ \\
$0.1-0.2$ & $9.099 \pm 0.007$ & $9.19 \pm 0.07$ \\
$0.2-0.3$ & $9.13 \pm 0.03$ & $9.034 \pm 0.006$ \\
$0.3-0.4$ & $9.08 \pm 0.05$ & $8.961 \pm 0.005$ \\
$0.4-0.5$ & $9.023 \pm 0.005$ & $9.009 \pm 0.007$ \\
$0.5-0.6$ & $9.05 \pm 0.01$ & $8.95 \pm 0.01$ \\
$0.6-0.7^{*}$ & $9.1855 \pm 0.0009$ & $9.022 \pm 0.009$ \\
$0.7-0.8^{*}$ & $9.0892 \pm 0.0004$ & $8.96 \pm 0.02$ \\
$0.8-0.9^{*}$ & $9.071 \pm 0.005$ & $8.939 \pm 0.005$ \\
$0.9-1.0^{*}$ & $9.087 \pm 0.004$ & $8.99 \pm 0.02$ \\
\hline
\end{tabular}

Table 4.3: Here we show the eccentricity-dependent limits on $\log _{10}(\mathcal{M})$ values for a 3C 66B-like source, as described in Section 4.3.2.2. We show the limits separately for NANOGrav 11-year and 12.5-year datasets. The stars next to the $e_{0}$ values indicate that we are not truly sensitive to these eccentricity values for the actual 3C 66B system (as discussed in Section 4.3.2.1); however, these results are valid for a 3C 66B-like target with orbital-period reference epochs close to the 2018-2020 era.

standard deviation of the resultant upper limit distribution as our error on that upper limit. As we wish to compare with previous limits (e.g. those by Jenet et al. 2004), it is appropriate to report an eccentricity-dependent limit on the chirp mass in the system.

We report the numerical results of this process in Table 4.3. It is important to note here that the choice of bin size does not greatly influence the results (particularly, as visible in the table, based on the lack of steep, fiducial dependence of the limits as a function of eccentricity).

There are several points of note about the limit results presented here. First, as expected, the N12 dataset in general places more stringent limits than the N11 data. The N11 data does not appear to have any clear dependence of the limits as a function of $e_{0}$, whereas the upper limits derived from the N12 data drops 
somewhat at high $e_{0}$ values; however, this difference appears to be negligible and we do not believe it to be a meaningful dependence. As expected, our limits on this 3C 66B-like source are significantly more stringent than the single-pulsar limits on mass-dependent eccentricity placed by Jenet et al. (2004).

The limit placed on the system using the N11 data allows us a direct comparison to the previous circular-only search for 3C 66B, as reported by Arzoumanian et al. (2020b). The "constant-value frequency prior" search performed in that work was similar to ours in many ways (e.g. it used the same base enterprise functions, an identical dataset with nearly identical noise models, referenced the period epoch to the last TOA, and did not include PSR J1713+0747), however had a few critical differences. Here we list only the differences mostly likely to have a potential impact on differences in the results derived from these searches:

1. Due to poor sampling in the N11 data when including PSR J0613-0200, additional noise modelling was included to account for co-variances of the circular search parameters with that pulsar's parameters.

2. The core code base that performed source evolution differed, and implicitly, they assumed the source eccentricity to be $e_{0}=0$.

3. The upper limit they placed on chirp mass was based on a uniform prior (as opposed to our log-uniform prior on $\mathcal{M})$.

4. Finally, their GW frequency was held fixed at 2/(1.05 years), as appropriate for a circular GW source, where as our binary orbital frequency was held fixed. 
The $95 \%$ limit reported by Arzoumanian et al. (2020b) for their constantvalue frequency prior on $3 \mathrm{C} 66 \mathrm{~B}$ was quoted as $\mathcal{M}<1.65 \pm 0.02 \times 10^{9} M_{\odot}$, or $\log _{10}(\mathcal{M})<9.217 \pm 0.005$. Comparing the first $\left(0 \geq e_{0} \geq\right)$ eccentricity bin in Table $4.3, \log _{10}(\mathcal{M})=9.043 \pm 0.007$, is not consistent with the upper limit results found by other searches as our uncertainty on our $95 \%$ upper limit is quite small. The lack of variance in our limits as a function of eccentricity don't account for the difference, and it is possible that some number of points in the above list could influence the marginal difference between the similar results from the same code. Points (1) and (4) likely have negligible impacts, however the direction of how these differences would skew the results are not obvious. Point (3) may be a likely source of discrepancy, as it is known that applying re-weighting to obtain a limit is sensitive to the lower bound placed on the prior; thus, this may impact some of the numerical difference we see here.

Still, the differences noted in the previous paragraph do not account for the distinct lack of samples in our results above a $\log _{10}\left(\mathcal{M} / M_{\odot}\right)$ around the Arzoumanian et al. limit of about 9.2 (as seen in the distributions in Figures 4.6 and 4.5). This brings us to point (2). In investigating differences between the circular and eccentric GW codes, we found that there appears to be a discrepancy of a factor of approximately two when directly comparing the amplitudes of signal-induced residuals predicted from the two codes; the waveform amplitudes predicted by GWecc appear to be a factor of two larger than the circular waveforms. While GWecc is not able to predict a perfectly circular waveform (thus, we compared circular waveforms to those with minimal values of $e_{0}=0.001,0.002,0.003$, the difference does 
not appear to be in the small amount of eccentricity required to run GWecc). A detailed investigation was unable to uncover the cause of this discrepancy, whether it accounts from a yet unidentified bug in the circular code, the eccentric code, or perhaps a factor of two error in the derivations of Susobhanan et al. (2020). Further investigation into the source of this discrepancy is beyond the scope of this work, however it is sufficient here to note that this could feasibly account for a more stringent limit being derived for our work than for that of Arzoumanian et al. (2020b). Additionally, we note that our injection-and-recovery self-check demonstrates that our pipeline can, at least, internally recover the correct parameters for an injected signal.

Finally, a possible reason for our more stringent limit values is potentially related to the parametric time parameter $\tau$ as defined in Susobhanan et al. (2020) and mentioned in Section 4.2.4. This parameter is used to determine the current state of a binary based on a given reference time. The code prevents sampling at values of $\tau<0$, implying (loosely) that the proposed binary may have merged within the observation window. This should not have been an issue due to our reference time being placed at the end of the dataset, however as $\tau$ is defined to be a conservative estimator for a merged system, this could possibly work to artificially restrict certain areas of the overall parameter space. Still, that we were able to demonstrate self-consistency of the code in the injected signal recovery (Section 4.3.1 and Figure 4.2) indicates that $\tau$ is not the likely cause of this inconsistency. 


\subsection{Conclusions}

In this work we implemented and performed tests on simulated data and did an eccentric GW search targeting the source 3C 66B using the N11 and N12 datasets. The purpose of this work was to determine if an eccentric GW search was plausible, and use a representative 3C 66B-like source as a test case for this method.

As this search is the first of its kind, there are some things to note in regards to nature of the search itself. From several sets of injection analyses done using the N11 and N12 datasets, we found that the N12 dataset sampled significantly better than the N11 dataset as there were fewer instances of the sampler getting trapped in small regions of the parameter space for extended periods of time. We attribute this to the fact that the N12 dataset has more pulsars with a 3-year timing baseline than the N11 dataset.

While the injection analysis proved semi-successful in the case described by Section 4.3.1, this was still accomplished by using a narrow uniform prior on $\log _{10}(\mathcal{M})$ and $e_{0}$. The restricted prior allowed the sampler to search the range that contained the injected signal, but this resulted in poor sampling of $e_{0}$. This is possibly due to the nature of $\tau_{0}$ (first mentioned in Section 4.2.4). $\tau_{0}$ is a function of $\mathcal{M}, e_{0}$, and $n$ and is used to determine if a binary has already merged within the timespan of the dataset. It is possible that certain pairings of $\mathcal{M}$ and $e_{0}$ do not satisfy the condition $\left(t-t_{0}\right) \ll t_{\text {merge }}$, resulting in the poor sampling of the restricted prior range used for the injection study described in Section 4.3.1.

Assuming the caveats discussed Section 4.3.2.2 are addressed, then it possi- 
ble to expand to an all-sky search for eccentric GWs. However, there are a couple of improvements that should be implemented into the current method to make an eccentric all-sky search plausible. The first is that the eccentric binary model implemented for this search does not account for black hole spin. At low eccentricities this is not a matter of concern, but that is not the case at high eccentricities as there is coupling between the spin and orbital eccentricity. Currently, there is a model being developed to incorporate spin (Dey et al. in prep). The second is that this search was done over a fixed orbital frequency. The code would need to be changed to search over GW frequency as that is customary with continuous GW searches.

This work has made it possible to perform targeted eccentric GW searches of NANOGrav data. With improvements to our search method it can be expanded to do an all-sky search for eccentric GWs. 


\section{Chapter 5}

\section{Conclusion}

Here we aim to summarize the work in this thesis and then look forward to the future of this field. Throughout this work, we sought to further the understanding of the formation of BBHs in the stellar-mass and super-massive regimes. We discussed the basics of GWs and how detections are made in the ground-based detector and PTA regimes. From there, we examined the meaning of eccentricity and the effect it has on GW signals, such as the dispersion of power among different frequency harmonics and faster evolution of the orbit with increased eccentricity. Then we discussed reasons as to why eccentric BBH sources haven't been detected in both the ground-based and PTA regimes. In the ground-based regime there is a lack of reliable eccentric waveform codes that make the use of matched-filtering to find eccentric BBH sources difficult. In the PTA-regime, previous eccentricity-based codes proved to be computationally inefficient, making it difficult to search for eccentric SMBHBs. This became the motivation to develop methods and code that could be used to search for eccentric BBH sources in both regimes. Finally, we developed methods to search for highly eccentric BBHs $(\mathrm{e} \geq 0.7)$ in the ground-based detector regime, in addition to non-circular SMBHBs in the PTA regime. Here we summarize the results of those methods and look to the future of detecting eccentric GWs. 


\subsection{Eccentric Burst Prior: Caveats and Future Improvements}

In Chapter 3, we presented the development and testing of an eccentric burst prior that could be implemented into a burst-search method to find bursts from a highly eccentric GW signal. This eccentric burst prior is based on the Newtonian Burst Model described by Loutrel \& Yunes (2017). We tested the eccentric burst prior on simulated data with the assumption that it is whitened and aLIGO single detector-like.

As noted in Section Section 3.4, the eccentric burst prior is seen to be more efficient at locating bursts for lower injected SNR values than with the use of a uniform prior, but this comes with some caveats. First, the eccentric burst prior is based on the Newtonian Burst model described by Loutrel \& Yunes (2017) and does not account for post-Newtonian effects, therefore making it difficult to use bursts closer to merger as this breaks our approximations.

Secondly, the forward and backward evolution of the bursts is not symmetric as a consequence of truncating the series whilst simplifying the equations for the backwards evolution. This mainly affects the choice of the anchor burst and results in a covariance between $M, \mathcal{M}$, and the choice of the anchor burst.

Thirdly, our analysis did not exhaustively explore the parameter space of masses, mass ratios, or eccentricities. Further exploration of the parameter space would be needed to properly define the transition region (the SNR region where the signal goes from undetected to detected). Lastly, our recovery of $M$ and $\mathcal{M}$ was very poor as a result of no merger information, and a lack of frequency evolution, 
in our specified time window.

Several improvements could be implemented to further advance the work done on the eccentric burst prior. The first is the addition of higher-order pN equations, which could improve precision in locating the bursts and allow for bursts closer to the merger phase to be used as anchor bursts. Next is a much more robust analysis on other sources to properly define the transition region. Finally, the exploration of using different wavelet waveform bases like chirplets (Millhouse et al., 2018) or hyperbolic encounters (Turner, 1977) that could be used to better describe the bursts in an eccentric GW signal. We leave these improvements for future work.

\subsection{Implementation of an Eccentric GW Search on PTA Data}

In Chapter 4 we implemented and performed an eccentric GW search over simulated and real data. While the injection-recovery analysis proved semi-successful as the injected signal utilizing parameters listed in Table 4.2 were recovered, it was done using a restricted uniform priors on $\log _{10}(\mathcal{M})$ and $e_{0}$. We also experienced poor sampling that could be combination of searching in a small region along with the nature of $\tau_{0}$. From our search on real data to find eccentric GW from a 3C 66B-like source, we concluded from our analysis that there were no detectable eccentric GWs present in the NANOGrav 11-year or 12.5-year datasets. This is further confirmed by the Bayes factors calculated from runs done on both datasets $\left(\mathcal{B}_{11}=0.305 \pm 0.103\right.$ and $\left.\mathcal{B}_{12.5}=0.327 \pm 0.105\right)$. From this work we were able to formulate a method such that once all the caveats of Section 4.3.2.2 are addressed, could be expanded 
to perform an all-sky search for eccentric GWs.

\subsubsection{Limits on Eccentricity and Chirp Mass}

We were also able to place eccentricity-based limits on $\log _{10}(\mathcal{M})$ for the NANOGrav 11-year and 12.5-year datasets. These values can be seen in Table 4.3. Our 95\% confidence value for the lowest eccentricity bin, $\log _{10}\left(\mathcal{M} / M_{\odot}\right)=9.043 \pm 0.007$, was found to be more conservative than the limit found by Arzoumanian et al. (2020b). Possible reasons as to why this is the case come from two points listed in Section 4.3.2.2: 1) the factor of two difference in the maximum timing residual amplitude when comparing eccentric vs circular residuals, and 2) the dependence on the lower bound of our prior due to reweighting.

\subsubsection{Future Improvements to Eccentric Searches on PTA Datasets}

As it stands, the work presented in Chapter 4 has enabled future searches for eccentric GWs on PTA datasets. While this is encouraging for future work with eccentric GWs, more work needs to be done in the modeling of eccentric SMBHB systems. Currently, the model utilized in our search does not incorporate black hole spin. For low eccentricities, there is little to no coupling between black hole spin and orbital eccentricity, but this is not the case for highly eccentric binaries. Efforts are being made to incorporate spin into the eccentric binary model (Dey et al. in prep) to hopefully be implemented in a similar fashion with the next few years. 


\subsection{The Future of Eccentric Gravitational-Wave Searches}

As we have learned throughout this work, the study of binary eccentricity is one of several key methods to better understanding the dynamics and environments of $\mathrm{BBH}$ systems in the stellar-mass and super-massive regimes. As each detector in different parts of the GW spectrum becomes more technologically advanced, and new facilities come online, raw detector sensitivity to these systems will improve and we can build a more complete picture of the GW spectrum, further developing our understanding of these systems. Here we focus on a discussion of the future prospects in this field.

\subsubsection{Ground-Based Detectors}

As things currently stand in terms of detection methods utilized by the LIGOVirgo-KAGRA Scientific Collaboration, a GW detection from an eccentric BBH system is unlikely. While the final upgrades to LIGO, known as A+, will make it more feasible to detect these systems, it will still be difficult; with the lack of reliable eccentric waveform models that contain an IMR and implemented into LALSuite, the use of matched-filtering to detect such systems is severely limited. Through the improvements of existing eccentric waveforms and the incorporation of newly developed eccentric waveforms that contain an IMR model, then a detection of GWs from an eccentric BBH system will be more plausible. Further development of the eccentric burst prior as noted in Section 5.1 may also contribute to the detection of highly eccentric BBH systems and therefore inform us about the formation of such 
systems.

For future third-generation ground-based detectors like Cosmic Explorer (Abbott et al., 2017d; Dwyer et al., 2015), the probability of a GW detection of an eccentric BBH is greater than that of aLIGO. With Cosmic Explorer's $40 \mathrm{~km}$ arms, the sensitivity greatly increases, making it capable of detecting binary systems at lower frequencies than that of aLIGO, Virgo, and KAGRA. A recent study looked into the computational cost of searching for eccentric binary neutron stars (BNS) and found that it would need, at most, half a year's worth of data to make a detectionm, if we assume the most pessimistic models (Nitz et al., 2019).

As the computational cost of matched-filter searches for Cosmic Explorer is on par with those done for aLIGO data, a detection of low-eccentricity BBH systems is more likely. A search done for eccentric BNS systems utilized 350,000 templates for an eccentricity range of $0 \leq e \leq 0.4$ (Lenon et al., 2021). For highly-eccentric $\mathrm{BBH}$ systems, the parameter space significantly increases, which in turn, expands the size of the template banks necessary to cover such a space. Therefore, the use of burst search methods to detect highly-eccentric BBH systems could be a more plausible way to detect such systems, making the work of Chapter 3 more applicable if implemented into a burst search method like Bayeswave.

\subsubsection{Pulsar Timing Arrays}

Recently, three worldwide pulsar timing arrays independently reported a shared noise process in their pulsar timing data streams that indicates a common red-noise 
signal affecting all pulsars (Arzoumanian et al., 2020c; Goncharov et al., 2021; Chen et al., 2021). It was hypothesized that this source could be the first hint of a GW background detection; however it has potential origins in some other noise source. So far, a gravitational-wave detection can not yet be confidently concluded because we do not yet have strong enough evidence that the noise correlations across the array obey the quadrupolar Hellings-and-Downs correlation expected of a GW (recall Figure 2.6 and Section 2.1.2).

If this signal does indicate the imminent confident detection of a gravitationalwave background, based on predictive modelling of the SMBHB population, it seems likely that this background may be consistent (in strain amplitude and spectral index) with the expectations of the background that should arise from the ensemble population of binary supermassive black holes (e.g. Sesana et al., 2008; Simon \& Burke-Spolaor, 2016; Siwek et al., 2020). Additionally, in modelling of the likely SMBHB population in the local universe, Mingarelli (2019) and others have demonstrated that it is likely that PTAs will have detected at least one continuous-wave source by the end of this decade. These promising predictions, and the current effort to combine the best and latest worldwide PTA data sets into a supremely sensitive "Data Release 3" for the IPTA, highlight the imminent need for the development of algorithms that efficiently search for realistic signals from SMBHBs, like the work presented in Chapter 4.

The methods and code we presented in Chapter 4 represent the first fully eccentric search on a PTA dataset. In addition, they represent the second-ever eccentric search for GW signatures from any binary on pulsar timing data at all 
(the first being the single-pulsar-search limit by Jenet et al. (2004)). Thus, we've added a critical ability to search over this important parameter when aiming to detect realistic SMBHBs in PTA datasets. Although this was a targeted search, this work can be expanded to search for eccentric signals across all sky locations and for targets at any frequency. As PTA datasets become more sensitive through better timing techniques, analyses, and increased timing baselines, more robust limits can be placed on eccentric signals which could eventually lead to a detection of eccentric GWs from a SMBHB. As detailed in our introduction, from the eccentricity values, any evolution-measurements of the future detections, and multi-messenger studies, we can learn more about the SMBHB environment and constrain theories that describe our current understanding of binary orbital evolution. 


\section{Bibliography}

Aasi, J., et al. 2015, Class. Quant. Grav., 32, 074001

Abbott, B., Abbott, R., Abbott, T., et al. 2019a, Physical Review X, 9, doi:10.1103/physrevx.9.031040. http://dx.doi.org/10.1103/PhysRevX.9. 031040

Abbott, B. P., et al. 2009, Rept. Prog. Phys., 72, 076901

—. 2016a, Phys. Rev. Lett., 116, 061102

—. 2016b, Phys. Rev. D, 93, 122003

—. 2017a, Phys. Rev. Lett., 119, 161101

—. 2017b, Phys. Rev. Lett., 119, 161101

—. 2017c, Astrophys. J. Lett., 848, L12

—. 2017d, Class. Quant. Grav., 34, 044001

—. 2019b, Astrophys. J. Lett., 882, L24

—. 2019c, Astrophys. J., 883, 149

—. 2020, Astrophys. J. Lett., 892, L3

Abbott, R., et al. 2021a, Phys. Rev. X, 11, 021053

—. 2021b, Astrophys. J. Lett., 915, L5

—. 2021c, Astrophys. J. Lett., 913, L7 
Acernese, F., et al. 2015, Class. Quant. Grav., 32, 024001

Aggarwal, K., Arzoumanian, Z., Baker, P. T., et al. 2019, Astrophys. J., 880, 116

Aggarwal, K., et al. 2019, Astrophys. J., 880, 2

Alam, M. F., et al. 2021a, Astrophys. J. Suppl., 252, 5

—. 2021b, Astrophys. J. Suppl., 252, 4

Alam, M. F., Arzoumanian, Z., Baker, P. T., et al. 2021, ApJS, 252, 4

Allen, B., Anderson, W. G., Brady, P. R., Brown, D. A., \& Creighton, J. D. E. 2012, Phys. Rev. D, 85, 122006

Amaro-Seoane, P., Sesana, A., Hoffman, L., et al. 2010, Mon. Not. Roy. Astron. Soc., 402, 2308

Amaro-Seoane, P., Audley, H., Babak, S., et al. 2017, arXiv e-prints, arXiv:1702.00786

Amaro-Seoane, P., et al. 2017, arXiv e-prints, arXiv:1702.00786

Armitage, P. J., \& Natarajan, P. 2005, ApJ, 634, 921

Arzoumanian, Z., Brazier, A., Burke-Spolaor, S., et al. 2018, ApJS, 235, 37

Arzoumanian, Z., Baker, P. T., Brazier, A., et al. 2020a, Astrophys. J., 900, 102

—. 2020b, Astrophys. J., 900, 102

Arzoumanian, Z., Baker, P. T., Blumer, H., et al. 2020c, ApJL, 905, L34 
Aso, Y., Michimura, Y., Somiya, K., et al. 2013, Phys. Rev. D, 88, 043007

Backer, D. C., Kulkarni, S. R., Heiles, C., Davis, M. M., \& Goss, W. M. 1982, Nature, 300, 615

Baker, P. T., \& Cheeseboro, B. D. 2021, ecc_prior, https://github.com/ paulthebaker/ecc_prior, ,

Banerjee, S. 2018a, Mon. Not. Roy. Astron. Soc., 473, 909

—. 2018b, Mon. Not. Roy. Astron. Soc., 481, 5123

Banerjee, S., Baumgardt, H., \& Kroupa, P. 2010, Mon. Not. Roy. Astron. Soc., 402, 371

Begelman, M. C., Blandford, R. D., \& Rees, M. J. 1980, Nature, 287, 307

Boetzel, Y., Susobhanan, A., Gopakumar, A., Klein, A., \& Jetzer, P. 2017, Phys. Rev. D, 96, 044011

Book, L. G., \& Flanagan, E. E. 2011, Phys. Rev. D, 83, 024024

Bramanti, D. 1968, Nature, 219, 47

Breivik, K., Rodriguez, C. L., Larson, S. L., Kalogera, V., \& Rasio, F. A. 2016, Astrophys. J. Lett., 830, L18

Burke-Spolaor, S., et al. 2019, Astron. Astrophys. Rev., 27, 5 
Caltech, L. 2016, A Brief History of LIGO, LIGO Laboratory \& California Institute of Technology, online: accessed 18 February 2021. https://www. ligo.caltech. edu/system/media_files/binaries/386/original/LIGOHistory.pdf

Cannon, K., Cariou, R., Chapman, A., et al. 2012, Astrophys. J., 748, 136

Cao, Z., \& Han, W.-B. 2017, Phys. Rev. D, 96, 044028

Cheeseboro, B., Baker, P., McWilliams, S., Lenon, A., \& LIGO Collaboration. 2017, in APS Meeting Abstracts, Vol. 2017, APS April Meeting Abstracts, T1.012

Cheeseboro, B. D., \& Baker, P. T. 2021, Phys. Rev. D, 104, 104016

Chen, S., Caballero, R. N., Guo, Y. J., et al. 2021, MNRAS, 508, 4970

Cornish, N. J., \& Littenberg, T. B. 2015, Classical and Quantum Gravity, 32, 135012. http://stacks.iop.org/0264-9381/32/i=13/a=135012

Cornish, N. J., Littenberg, T. B., Bécsy, B., et al. 2021, Phys. Rev. D, 103, 044006

Cuadra, J., Armitage, P. J., Alexander, R. D., \& Begelman, M. C. 2009, MNRAS, 393,1423

Davis, M. M., Taylor, J. H., Weisberg, J. M., \& Backer, D. C. 1985, Nature, 315, 547

Demorest, P. B., Ferdman, R. D., Gonzalez, M. E., et al. 2013, ApJ, 762, 94

Dickey, J. M. 1971, The Annals of Mathematical Statistics, 42, 204. http://www. jstor.org/stable/2958475 
D’Orazio, D. J., \& Samsing, J. 2018, MNRAS, 481, 4775

Dwyer, S., Sigg, D., Ballmer, S. W., et al. 2015, Phys. Rev. D, 91, 082001. https: //link.aps.org/doi/10.1103/PhysRevD.91.082001

East, W. E., McWilliams, S. T., Levin, J., \& Pretorius, F. 2013, Phys. Rev. D, 87, 043004

Ellis, J., \& van Haasteren, R. 2017, jellis18/PTMCMCSampler: Official Release, , , doi:10.5281/zenodo.1037579. https://doi.org/10.5281/zenodo. 1037579

Ellis, J. A., Siemens, X., \& Creighton, J. D. E. 2012, Astrophys. J., 756, 175

Ellis, J. A., Vallisneri, M., Taylor, S. R., \& Baker, P. T. 2020, ENTERPRISE: Enhanced Numerical Toolbox Enabling a Robust PulsaR Inference SuitE, Zenodo, , , doi:10.5281/zenodo.4059815. https://doi.org/10.5281/zenodo.4059815

Finn, L. S., \& Lommen, A. N. 2010, Astrophys. J., 718, 1400

Fitts, A., et al. 2017, Mon. Not. Roy. Astron. Soc., 471, 3547

Foster, R. S., \& Backer, D. C. 1990, Astrophys. J., 361, 300

García, F., Bunzel, A. S., Chaty, S., Porter, E., \& Chassande-Mottin, E. 2021, Astron. Astrophys., 649, A114

Gayathri, V., Healy, J., Lange, J., et al. 2020, arXiv e-prints, arXiv:2009.05461

Gelman, A., Carlin, J. B., Stern, H. S., \& Rubin, D. B. 2014, Bayesian Data Analysis, 3rd edn., CRC Texts in Statistical Science (Chapman \& Hall) 
Goncharov, B., Shannon, R. M., Reardon, D. J., et al. 2021, ApJL, 917, L19

Haiman, Z., Kocsis, B., \& Menou, K. 2009, ApJ, 700, 1952

Hazboun, J. S., Romano, J. D., \& Smith, T. L. 2019, Phys. Rev. D, 100, 104028

Hazboun, J. S., Simon, J., Taylor, S. R., et al. 2020, Astrophys. J., 890, 108

Hellings, R. W., \& Downs, G. S. 1983, Astrophys. J. Lett., 265, L39

Hewish, A., Bell, S. J., Pilkington, J. D. H., Scott, P. F., \& Collins, R. A. 1968, Nature, 217, 709

Hinder, I., Kidder, L. E., \& Pfeiffer, H. P. 2018, Phys. Rev. D, 98, 044015

Hobbs, G. B., Edwards, R. T., \& Manchester, R. N. 2006, MNRAS, 369, 655

Huerta, E., et al. 2017, Phys. Rev. D, 95, 024038

Huerta, E. A., Kumar, P., McWilliams, S. T., O’Shaughnessy, R., \& Yunes, N. 2014, Phys. Rev. D, 90, 084016

Huerta, E. A., et al. 2018, Phys. Rev. D, 97, 024031

Iguchi, S., Okuda, T., \& Sudou, H. 2010, Astrophys. J. Lett., 724, L166

Ivanov, P. B., Papaloizou, J. C. B., \& Polnarev, A. G. 1999, Mon. Not. Roy. Astron. Soc., 307,79

Jenet, F. A., Lommen, A., Larson, S. L., \& Wen, L. 2004, Astrophys. J., 606, 799

Jenet, F. A., \& Romano, J. D. 2015, American Journal of Physics, 83, 635 
Jenet, F. A., Hobbs, G. B., van Straten, W., et al. 2006, Astrophys. J., 653, 1571

Klimenko, S., Yakushin, I., Mercer, A., \& Mitselmakher, G. 2008, Classical and Quantum Gravity, 25, 114029

Kocsis, B., \& Sesana, A. 2011, MNRAS, 411, 1467

Kormendy, J., \& Richstone, D. 1995, Annual Rev. of Astron. and Astrophys., 33, 581

Lam, M. T. 2016, PhD thesis, Cornell University

Lam, M. T., Ellis, J. A., Grillo, G., et al. 2018, Astrophys. J., 861, 132

Latif, M. A., \& Ferrara, A. 2016, Publications of the Astron. Soc. of Australia, 33, $\mathrm{e} 051$

Lee, K. J., Wex, N., Kramer, M., et al. 2011, Monthly Notices of the Royal Astronomical Society, 414, 3251. https://doi.org/10.1111/j.1365-2966.2011. $18622 . \mathrm{x}$

Lenon, A. K., Brown, D. A., \& Nitz, A. H. 2021, Phys. Rev. D, 104, 063011. https://link.aps.org/doi/10.1103/PhysRevD.104.063011

LIGO Scientific Collaboration. 2018, LIGO Algorithm Library - LALSuite, free software (GPL), , doi:10.7935/GT1W-FZ16

Littenberg, T. B., \& Cornish, N. J. 2015, Phys. Rev. D, 91, 084034. https://Iink. aps.org/doi/10.1103/PhysRevD.91.084034 
Lorimer, D. R. 2001, Living Reviews in Relativity, 4, 5

Lorimer, D. R., \& Kramer, M. 2012, Handbook of Pulsar Astronomy

Loutrel, N. 2020, Class. Quant. Grav., 37, 075008

Loutrel, N., \& Yunes, N. 2017, Classical and Quantum Gravity, 34, 135011. http: //stacks.iop.org/0264-9381/34/i=13/a=135011

Lower, M. E., Thrane, E., Lasky, P. D., \& Smith, R. 2018, Phys. Rev. D, 98, 083028

Madison, D. R., Cordes, J. M., Arzoumanian, Z., et al. 2019, Astrophys. J., 872, 150

Mapelli, M. 2020, Formation Channels of Single and Binary Stellar-Mass Black Holes, ed. C. Bambi, S. Katsanevas, \& K. D. Kokkotas (Singapore: Springer Singapore), 1-65. https://doi.org/10.1007/978-981-15-4702-7_16-1

Memmesheimer, R.-M., Gopakumar, A., \& Schaefer, G. 2004, Phys. Rev. D, 70, 104011

Mikkola, S., \& Valtonen, M. J. 1992, Monthly Notices of the Royal Astronomical Society, 259, 115. https://doi.org/10.1093/mnras/259.1.115

Millhouse, M., Cornish, N. J., \& Littenberg, T. 2018, Phys. Rev. D, 97, 104057

Mingarelli, C. M. F. 2019, Nature Astronomy, 3, 8

Moore, B., Favata, M., Arun, K. G., \& Mishra, C. K. 2016, Phys. Rev., D93, 124061

Moore, B., \& Yunes, N. 2019, Class. Quant. Grav., 36, 185003 
Moore, C. J., Cole, R. H., \& Berry, C. P. L. 2015, Classical and Quantum Gravity, 32,015014

Nagar, A., Bonino, A., \& Rettegno, P. 2021, Phys. Rev. D, 103, 104021

Nitz, A. H., Capano, C. D., Kumar, S., et al. 2021, ArXiv e-print, arXiv:2105.09151

Nitz, A. H., Lenon, A., \& Brown, D. A. 2019, Astrophys. J., 890, 1

Nitz, A. H., Dent, T., Davies, G. S., et al. 2020, Astrophys. J., 891, 123

Olejak, A., Belczynski, K., \& Ivanova, N. 2021, ArXiv e-print, arXiv:2102.05649

O’Shea, E., \& Kumar, P. 2021, arXiv e-prints, arXiv:2107.07981

Paczynski, B. 1976, in Structure and Evolution of Close Binary Systems, ed. P. Eggleton, S. Mitton, \& J. Whelan, Vol. 73, 75

Paliya, V. S., et al. 2019, arXiv:1903.06106

Perera, B. B. P., et al. 2019, Mon. Not. Roy. Astron. Soc., 490, 4666

Peters, P. C. 1964, Phys. Rev., 136, B1224

Peters, P. C., \& Mathews, J. 1963, Phys. Rev., 131, 435

Porter, E. K., \& Sesana, A. 2010, arXiv e-prints, arXiv:1005.5296

Quinlan, G. D. 1996, New Astronomy, 1, 35

Quinlan, G. D. 1996, New Astronomy, 1, 35. https://www.sciencedirect.com/ science/article/pii/S1384107696000036 
Rees, M. J. 1984, Annual Rev. of Astron. and Astrophys., 22, 471

Rodriguez, C. L., Amaro-Seoane, P., Chatterjee, S., et al. 2018, Phys. Rev. D, 98, 123005

Roedig, C., Dotti, M., Sesana, A., Cuadra, J., \& Colpi, M. 2011, MNRAS, 415, 3033

Roedig, C., \& Sesana, A. 2012, in Journal of Physics Conference Series, Vol. 363, Journal of Physics Conference Series, 012035

Romero-Shaw, I. M., Lasky, P. D., \& Thrane, E. 2019, Mon. Not. Roy. Astron. Soc., 490,5210

—. 2021, arXiv e-prints, arXiv:2108.01284

Romero-Shaw, I. M., Lasky, P. D., Thrane, E., \& Bustillo, J. C. 2020, Astrophys. J. Lett., 903, L5

Ryden, B., \& Peterson, B. M. 2020, Foundations of astrophysics (Cambridge University Press)

Sabater, J. 2015, wquantiles, https://github.com/nudomarinero/wquantiles, ,

Samsing, J., \& D’Orazio, D. J. 2018, MNRAS, 481, 5445

Scalo, J. M. 1986, Fundamentals of cosmic physics, 11, 1

Sesana, A. 2010, ApJ, 719, 851

Sesana, A., Gualandris, A., \& Dotti, M. 2011, Mon. Not. Roy. Astron. Soc., 415, 35 
Sesana, A., Haardt, F., \& Madau, P. 2006, Astrophys. J., 651, 392

Sesana, A., \& Vecchio, A. 2010, Phys. Rev. D, 81, 104008

Sesana, A., Vecchio, A., \& Colacino, C. N. 2008, MNRAS, 390, 192

Shannon, R. M., Ravi, V., Lentati, L. T., et al. 2015, Science, 349, 1522

Simon, J., \& Burke-Spolaor, S. 2016, ApJ, 826, 11

Siwek, M. S., Kelley, L. Z., \& Hernquist, L. 2020, MNRAS, 498, 537

Sudou, H., Iguchi, S., Murata, Y., \& Taniguchi, Y. 2003, Science, 300, 1263

Sudou, H., Iguchi, S., \& Zhao, G.-Y. 2017, Astrophys. J., 841, 103

Susobhanan, A. 2020, GWecc: Calculator for pulsar timing array signals due to eccentric supermassive binaries, , , ascl:2002.013

Susobhanan, A., Gopakumar, A., Hobbs, G., \& Taylor, S. R. 2020, Phys. Rev. D, 101,043022

Tanay, S., Haney, M., \& Gopakumar, A. 2016, Phys. Rev. D, 93, 064031

Taylor, S. R., Huerta, E. A., Gair, J. R., \& McWilliams, S. T. 2016, Astrophys. J., 817,70

Taylor, S. R., Simon, J., \& Sampson, L. 2017, Phys. Rev. Lett., 118, 181102

Tiwari, S., Achamveedu, G., Haney, M., \& Hemantakumar, P. 2019, Phys. Rev. D, 99, 124008 
Turner, M. 1977, ApJ, 216, 610

Unnikrishnan, C. S. 2013, Int. J. Mod. Phys. D, 22, 1341010

Usman, S. A., Nitz, A. H., Harry, I. W., et al. 2016, Classical and Quantum Gravity, 33,215004

Vallisneri, M., Ellis, J. A., van Haasteren, R., et al. 2013, libstempo, https:// github.com/vallis/libstempo, ,

Vogelsberger, M., Genel, S., Springel, V., et al. 2014, Nature, 509, 177

Wahlquist, H. 1987, Gen. Rel. Grav., 19, 1101

Wu, S., Cao, Z., \& Zhu, Z.-H. 2020, Mon. Not. Roy. Astron. Soc., 495, 466

Zevin, M., Romero-Shaw, I. M., Kremer, K., Thrane, E., \& Lasky, P. D. 2021, arXiv e-prints, arXiv:2106.09042

Zhao, G. Y., Chen, Y. J., Shen, Z. Q., et al. 2011, Journal of Astrophysics and Astronomy, 32, 61

Zhao, G.-Y., Jung, T., Dodson, R., Rioja, M., \& Sohn, B. W. 2015, Publication of Korean Astronomical Society, 30, 629

Zhu, X.-J., Cui, W., \& Thrane, E. 2019, Mon. Not. Roy. Astron. Soc., 482, 2588

Zhu, X.-J., Wen, L., Hobbs, G., et al. 2015, Mon. Not. Roy. Astron. Soc., 449, 1650 